\title{
Semisimple characters for $p$-adic classical groups
}

\author{
Shaun Stevens*
}

6 May 2004

\begin{abstract}
Let $G$ be a unitary, symplectic or orthogonal group over a non-archimedean local field of residual characteristic different from 2, considered as the fixed point subgroup in a general linear group $\widetilde{G}$ of an involution. Following [7] and [13, we generalize the notion of a semisimple character for $\widetilde{G}$ and for $G$. In particular, following the formalism of [4], we show that these semisimple characters have certain functorial properties. Finally, we show that any positive level supercuspidal representation of $G$ contains a semisimple character.
\end{abstract}

\section{Introduction}

Let $F$ be a non-archimedean local field and let $\widetilde{G}=G L(N, F)$. One of the main ingredients in the description of the admissible dual of $\widetilde{G}$ by Bushnell and Kutzko ([6], [7]) is the notion of simple characters: these are arithmetically defined characters of certain compact open subgroups of $\widetilde{G}$. To obtain all the irreducible supercuspidal representations of $\widetilde{G}$ in [6], there are three main steps: first, to show that these simple characters have some rather remarkable properties of functoriality (and it turns out that they even have such properties when the dimension $N$ is allowed to vary (see 6], [10]) and similarly for the base field $F$ (see 4, [5] and sequels); second, to show that any irreducible supercuspidal representation of $\widetilde{G}$ contains a simple character $\theta$ of a group denoted $H^{1}$; and finally, to find the representations of the normalizer in $\widetilde{G}$ of $\theta$ which contain $\theta$.

The purpose of this paper is to prove results analogous to the first two steps for unitary, symplectic and orthogonal groups $G$, in the case where the residual characteristic of $F$ is not 2. To do this, we must first generalize the notion of simple characters to what we call semisimple characters of $\widetilde{G}$ and $G$. (There is a definition of semisimple characters in [13] but, as is remarked there, it is not sufficiently general.) We calculate the intertwining of these characters and demonstrate some functorial properties. Finally, we show that any irreducible supercuspidal representation of $G$ contains a semisimple character of a group denoted $H_{-}^{1}$.

Now we give a more detailed description of the results obtained. As above, let $F$ be a nonarchimedean local field of residual characteristic different from 2, equipped with a galois involution with fixed field $F_{0}$ (where we allow the possibility $F_{0}=F$ ). Let $V$ be an $N$-dimensional $F$-vector space, $\widetilde{G}=\operatorname{Aut}_{F}(V) \simeq G L(N, F)$ and let $G$ be the group of fixed points in $\widetilde{G}$ of an involution $\sigma$ defined by a nondegenerate hermitian or skew-hermitian form on $V$; so $G$ is a unitary, symplectic or orthogonal group defined over $F_{0}$. We also set $A_{-}=\operatorname{Lie} G \subset A=\operatorname{End}_{F}(V) \simeq \mathbb{M}(N, F)$.

\footnotetext{
*The research for this paper was partially funded by the EU network TMR "Arithmetic Algebraic Geometry" and by the Sonderforschungsbereich 478 "Geometrische Strukturen in der Mathematik", Münster.

Mathematics Subject Classification (2000): 22E50
} 
The simple characters for $\widetilde{G}$ are parametrized by triples $(\beta, \Lambda, m)$ consisting of: an element $\beta \in A$ which generates a field extension $E$ over $F$, with the technical condition $k_{F}(\beta)<0$; an $\mathfrak{o}_{E}$-lattice sequence $\Lambda$ of period $e(\Lambda)$ in $V$, where $\mathfrak{o}_{E}$ is the ring of integers of $E$; and an integer $m$ with $0 \leq m<k_{F}(\beta) e(\Lambda)$. (See $\$ 1.2$ and $\$ 2.1$ for some explanations of the terms here.) To such a triple is associated a compact open subgroup $H^{m+1}=H^{m+1}(\beta, \Lambda)$ of $\widetilde{G}$ and a finite set $\mathcal{C}(\Lambda, m, \beta)$ of simple characters. If $\left(\beta, \Lambda^{\prime}, m^{\prime}\right)$ is another such triple with $\left\lfloor\frac{m}{e(\Lambda)}\right\rfloor=\left\lfloor\frac{m^{\prime}}{e\left(\Lambda^{\prime}\right)}\right\rfloor$, the functoriality properties mentioned above give a canonical bijection between the sets $\mathcal{C}(\Lambda, m, \beta)$ and $\mathcal{C}\left(\Lambda^{\prime}, m^{\prime}, \beta\right)$. (Here, $\lfloor q\rfloor$ denotes the greatest integer less than or equal to $q$. )

If we have a self-dual triple, that is $\beta \in A_{-}$and $\Lambda$ is a self-dual lattice sequence, then the group $H^{m+1}$ and the set of simple characters are fixed by the involution $\sigma$ and we can define the set $\mathcal{C}_{-}(\Lambda, m, \beta)$ of simple characters for $G$ to be obtained by restricting to $H_{-}^{m+1}:=H^{m+1} \cap G$ the simple characters in $\mathcal{C}(\Lambda, m, \beta)$. Equivalently, and often more usefully, they are the transfers of those simple characters fixed by $\sigma$ under the Glauberman correspondence (note that $H^{m+1}$ is a pro- $p$ group, with $p \neq 2$ ). The intertwining of simple characters for $G$ can be calculated by intersection, from the intertwining in $\widetilde{G}$ (cf. [13], [12]). Moreover, if $\left(\beta, \Lambda^{\prime}, m^{\prime}\right)$ is another such triple with $\left\lfloor\frac{m}{e(\Lambda)}\right\rfloor=\left\lfloor\frac{m^{\prime}}{e\left(\Lambda^{\prime}\right)}\right\rfloor$, the canonical bijection above commutes with action of $\sigma$ (Proposition 2.12 and so induces a bijection between $\mathcal{C}_{-}(\Lambda, m, \beta)$ and $\mathcal{C}_{-}\left(\Lambda^{\prime}, m^{\prime}, \beta\right)$.

In [13, the notion of simple character is generalized to that of semisimple character, and in $\$ 3$ we generalize it further (cf. [13] $\S 5$ Remark 2). We take now a triple $(\beta, \Lambda, m)$ where $\beta$ generates a sum of fields $E=\oplus_{i} E_{i}$; this gives us decompositions $\beta=\sum_{i} \beta_{i}$ (with $E_{i}=F\left[\beta_{i}\right]$ ) and $V=\oplus_{i} V^{i}$, with each $V^{i}$ an $E_{i}=F\left[\beta_{i}\right]$-vector space, and we also require that $\Lambda$ decompose as a direct sum $\oplus_{i} \Lambda^{i}$, with each $\Lambda^{i}$ an $\mathfrak{o}_{E_{i}}$-lattice chain. (Again, there is a technical condition which can be written $k_{0}(\beta, \Lambda)<0$.)

For each $i$, the triple $\left(\beta_{i}, \Lambda_{i}, m\right)$ determines a compact open subgroup $H^{m+1}\left(\beta_{i}, \Lambda_{i}\right)$ and a set of simple characters as above. Analogously to the definitions in the simple case, we can then define a compact open subgroup $H^{m+1}(\beta, \Lambda)$, whose restriction to the Levi subgroup $M$ of $\widetilde{G}$ determined by the decomposition $V=\oplus_{i} V^{i}$ is the product of the groups $H^{m+1}\left(\beta_{i}, \Lambda_{i}\right)$. The set of semisimple characters $\mathcal{C}(\Lambda, m, \beta)$ for $\widetilde{G}$ then consists of characters of $H^{m+1}(\beta, \Lambda)$ which restrict to simple characters on each $H^{m+1}\left(\beta_{i}, \Lambda_{i}\right)$ and are trivial elsewhere (there must also be some compatibility conditions between these simple characters).

In 33.3 we calculate the intertwining of such a semisimple character and in $\$ 3.5$ we show that semisimple characters possess the same transfer properties as simple characters. Finally, in 3.6 , we consider the situation when, for each $i$, the triple $\left(\beta_{i}, \Lambda_{i}, m\right)$ is self-dual (note that, in this situation, $M \cap G$ is not a Levi subgroup of $G$ ); using Glauberman's correspondence again, all the results pass over to $G$, as in the simple case, and we get a set $\mathcal{C}_{-}(\Lambda, m, \beta)$ of semisimple characters for $G$.

Our main result is Theorem 5.1,

Any positive-level irreducible supercuspidal representation of $G$ contains a semisimple character $\theta \in \mathcal{C}_{-}(\Lambda, 0, \beta)$, for some self-dual semisimple triple $(\beta, \Lambda, 0)$.

The proof is very much along the lines of [6] (8.1.5), though the geometry causes some extra complications. We know already, from [14] $\S 1.3$, that such a representation $\pi$ contains a semisimple stratum, that is, a semisimple character in some $\mathcal{C}_{-}(\Lambda, n-1, \beta)$, where $n=-\nu_{\Lambda}(\beta)$. The idea is to "refine" this character, that is, to find a related semisimple character of lower level which is also contained in $\pi$. We illustrate the first step of the process here. 
The representation $\pi$ must contain a character of the group $H_{-}^{n-1}(\beta, \Lambda)$; comparing this to the semisimple characters of $H_{-}^{n-1}(\beta, \Lambda)$ gives rise to a derived stratum in the centralizer of $\beta$. There are three possibilities now: if the stratum is non-fundamental (see Definition 1.2), by changing $\Lambda$, we can obtain a semisimple character of lower level; if the stratum is $G$-split (see Definition 1.3), we show (Theorem 4.9) that $\pi$ has a non-zero Jacquet module, which contradicts the supercuspidality of $\pi$; otherwise, by replacing both $\beta$ and $\Lambda$ we obtain a semisimple character of lower level.

To prove the theorem, we iterate this, noting that we can bound the denominator of the level of the semisimple characters we consider so the process will terminate.

\section{Preliminaries}

We refer the reader to [6], [7, [12], [13] for more details on the results recalled in this section.

\section{$1.1 \quad$ Notations}

Let $F$ be a non-archimedean local field equipped with a galois involution ${ }^{-}$with fixed field $F_{0}$; we allow the possibility $F=F_{0}$. Let $\mathfrak{o}_{F}$ be the ring of integers of $F, \mathfrak{p}_{F}$ its maximal ideal and $k_{F}=\mathfrak{o}_{F} / \mathfrak{p}_{F}$ the residue field; we assume throughout that the residual characteristic $p:=$ char $k_{F}$ is not 2 . We denote by $\mathfrak{o}_{0}, \mathfrak{p}_{0}, k_{0}$ the same objects in $F_{0}$, and will use similar notation for any nonarchimedean local field. We fix a uniformizer $\varpi_{F}$ of $F$ such that $\overline{\varpi_{F}}=-\varpi_{F}$ if $F / F_{0}$ is ramified, $\varpi_{F}=\varpi_{F}$ otherwise. We put $\varpi_{0}=\varpi_{F}^{2}$ if $F / F_{0}$ is ramified, $\varpi_{0}=\varpi_{F}$ otherwise; so $\varpi_{0}$ is a uniformizer of $F_{0}$.

Let $V$ be an $N$-dimensional vector space over $F$, equipped with a nondegenerate $\epsilon$-hermitian form, with $\epsilon= \pm 1$. We put $A=\operatorname{End}_{F} V$ and denote by ${ }^{-}$the adjoint (anti-)involution on $A$ induced by $h$. Set also $\widetilde{G}=\operatorname{Aut}_{F} V$ and let $\sigma$ be the involution given by $g \mapsto \bar{g}^{-1}$, for $g \in \widetilde{G}$. We also have an action of $\sigma$ on the Lie algebra $A$ given by $a \mapsto-\bar{a}$, for $a \in A$ (this is the differential of the action on $\widetilde{G})$. We put $\Sigma=\{1, \sigma\}$, where 1 acts as the identity on both $\widetilde{G}$ and $A$.

We put $G=\widetilde{G}^{\Sigma}=\{g \in \widetilde{G}: h(g v, g w)=h(v, w)$ for all $v, w \in V\}$, a unitary, symplectic or orthogonal group over $F_{0}$, and $A_{-}=A^{\Sigma} \simeq \operatorname{Lie} G$. In general, for $S$ a subset of $A$, we will write $S_{-}$ or $S^{-}$for $S \cap A_{-}$, and, for $H$ a subgroup of $\widetilde{G}$, we will write $H_{-}$or $H^{-}$for $H \cap G$.

Let $\psi_{0}$ be a character of the additive group of $F_{0}$, with conductor $\mathfrak{p}_{0}$. Then we put $\psi_{F}=\psi_{0} \circ \operatorname{tr}_{F / F_{0}}$; since $p \neq 2, F / F_{0}$ is at worst tamely ramified so $\psi_{F}$ is a character of the additive group of $F$ with conductor $\mathfrak{p}_{F}$. For $S$ an $\mathfrak{o}_{F}$-lattice in $A$, we put $S^{*}=\left\{x \in A: \psi_{A}(x S)=1\right\}$. If $S$ is fixed by $\sigma$, then we have $S^{*} \cap A_{-}=\left\{x \in A_{-}: \psi_{A}\left(x S_{-}\right)=1\right\}$.

We will also frequently have a decomposition $V=\bigoplus_{i=1}^{l} V^{i}$. Then, for $1 \leq i \leq l$, we denote by $\mathbf{1}^{i}$ the projection $V \rightarrow V^{i}$ with kernel $\bigoplus_{j \neq i} V^{j}$ and put $A^{i j}=\mathbf{1}^{i} A \mathbf{1}^{j}=\operatorname{Hom}_{F}\left(V^{j}, V^{i}\right)$. Then we will use the block decomposition

$$
A=\left(\begin{array}{ccc}
A^{11} & \cdots & A^{1 l} \\
\vdots & \ddots & \vdots \\
A^{l 1} & \cdots & A^{l l}
\end{array}\right) .
$$

We also put $A_{u}=\bigoplus_{1 \leq i<j \leq l} A^{i j}, A_{l}=\bigoplus_{1 \leq j<i \leq l} A^{i j}$ and $\mathcal{M}=\bigoplus_{i=1}^{l} A^{i i}$; and $N_{u}=1+A_{u}$, $N_{l}=1+A_{l}, M=\mathcal{M}^{\times}, P_{u}=M N_{u}, P_{l}=M N_{l}$. 
Finally, for $r \in \mathbb{R}$, we denote: by $\lceil r\rceil$ the smallest integer greater than or equal to $r$; by $r+$ the smallest integer strictly greater than $r$; by $\lfloor r\rfloor$ the greatest integer less than or equal to $r$; and by $r$ - the greatest integer strictly less than $r$.

\section{$1.2 \quad$ Strata}

Recall, from [7] $\S 2$, that an $\mathfrak{o}_{F}$-lattice sequence in $V$ is a function $\Lambda$ from $\mathbb{Z}$ to the set of $\mathfrak{o}_{F}$-lattices in $V$ such that

(i) $\Lambda(k) \subset \Lambda(j)$, for $k \geq j$;

(ii) there exists a positive integer $e=e\left(\Lambda \mid \mathfrak{o}_{F}\right)$, called the $\mathfrak{o}_{F}$-period of $\Lambda$, such that $\varpi_{F} \Lambda(k)=$ $\Lambda(k+e)$, for all $k \in \mathbb{Z}$.

A lattice sequence is called strict if $\Lambda(k) \neq \Lambda(j)$, for $k \neq j$ (so $\Lambda$ is really just an $\mathfrak{o}_{F}$-lattice chain - see e.g. [6] (1.1.1)).

For $L$ an $\mathfrak{o}_{F}$-lattice in $V$, we put $L^{\#}=\left\{v \in V: h(v, L) \subset \mathfrak{p}_{F}\right\}$. Then we call an $\mathfrak{o}_{F}$-lattice sequence $\Lambda$ self-dual if there exists $d \in \mathbb{Z}$ such that $\Lambda(k)^{\#}=\Lambda(d-k)$, for all $k \in \mathbb{Z}$. Without changing any of the objects associated to a self-dual $\mathfrak{o}_{F}$-lattice sequence $\Lambda$ (except for a scale of the indices), we may (and do) normalize all self-dual lattice sequences $\boldsymbol{\Lambda}$ so that $\boldsymbol{d}=\mathbf{1}$ (see [14] $\S 2$ ).

There is also a well-defined notion of the direct sum of lattice sequences (see [7] $\S 2$ for the definition and some properties). The direct sum of self-dual lattice sequences is itself self-dual, by the assumption $d=1$.

Associated to an $\mathfrak{o}_{F}$-lattice sequence $\Lambda$ in $V$, we have a decreasing filtration $\left\{\mathfrak{a}_{n}(\Lambda): n \in \mathbb{Z}\right\}$ of $A$ by $\mathfrak{o}_{F}$-lattices; $\mathfrak{a}_{0}$ is a hereditary $\mathfrak{o}_{F}$-order in $A$ and $\mathfrak{a}_{1}$ is its Jacobson radical. As in [7], we will allow the indices in the filtration to be real numbers, by putting $\mathfrak{a}_{n}=\mathfrak{a}_{\lceil n\rceil}$, for $n \in \mathbb{R}$. Note also that, for $n \in \mathbb{Z}$, the integers $\left\lfloor\frac{n}{2}\right\rfloor+1$ and $\left\lfloor\frac{n+1}{2}\right\rfloor$ often appear in [6], [7] etc.; with the notation as here, we have

$$
\mathfrak{a}_{\left\lfloor\frac{n}{2}\right\rfloor+1}=\mathfrak{a}_{\frac{n}{2}+}, \quad \text { and } \quad \mathfrak{a}_{\left\lfloor\frac{n+1}{2}\right\rfloor}=\mathfrak{a}_{\frac{n}{2}} .
$$

The filtration on $A$ also gives rise to a valuation $\nu_{\Lambda}$ on $A$, with $\nu_{\Lambda}(0)=+\infty$.

If $\Lambda$ is self-dual, then each $\mathfrak{a}_{n}(\Lambda)$ is fixed by $\sigma$ and $\mathfrak{a}_{n}^{-}=\mathfrak{a}_{n}^{-}(\Lambda)=\mathfrak{a}_{n}(\Lambda) \cap A_{-}$gives a filtration of $A_{-}$by $\mathfrak{o}_{F}$-lattices. Moreover, $\nu_{\Lambda}$ is fixed by $\sigma$.

Given an $\mathfrak{o}_{F}$-lattice sequence $\Lambda$, we also put $U=U(\Lambda)=\mathfrak{a}_{0}(\Lambda)^{\times}$, a compact open subgroup of $\widetilde{G}$, and $U_{n}=U_{n}(\Lambda)=1+\mathfrak{a}_{n}(\Lambda)$, for $n>0$, a filtration of $U(\Lambda)$ by normal subgroups. For $n>0$, we have a group isomorphism $\mathfrak{a}_{n} / \mathfrak{a}_{n+} \stackrel{\sim}{\rightarrow} U_{n} / U_{n+}$ induced by $x \mapsto 1+x$.

If $\Lambda$ is self-dual, then $U, U_{n}$ are fixed by $\sigma$ and we put $P=P(\Lambda)=U(\Lambda)^{\Sigma}=U(\Lambda) \cap G$, a compact open subgroup of $G$, with a filtration of $P(\Lambda)$ by normal subgroups $P_{n}=P_{n}(\Lambda)=U_{n}(\Lambda)^{\Sigma}=$ $U_{n}(\Lambda) \cap G$, for $n \geq 1$. As before, for $n \geq 1$ we have a group isomorphism $\mathfrak{a}_{n}^{-} / \mathfrak{a}_{n+}^{-} \stackrel{\sim}{\rightarrow} P_{n} / P_{n+}$. We also have, for $n>0$, a bijection $\mathfrak{a}_{n}^{-} \rightarrow P_{n}$ given by the Cayley map $x \mapsto C(x)=\left(1+\frac{x}{2}\right)\left(1-\frac{x}{2}\right)^{-1}$, which is equivariant under conjugation by $P$.

We define the normalizer $\mathfrak{K}(\Lambda)$ to be $\mathfrak{K}(\Lambda)=\bigcap_{n \geq 0} N_{\widetilde{G}}\left(U_{n}(\Lambda)\right)$, where $N_{\widetilde{G}}$ denotes the normalizer in $\widetilde{G}$. Note that, if $x \in \mathfrak{K}(\Lambda)$, then $\nu_{\Lambda}(x)=-\nu_{\Lambda}\left(x^{-1}\right)$ (see [7] (3.4)). On the other hand, if $x \in G$ and $\Lambda$ is self-dual then $\nu_{\Lambda}(x)=\nu_{\Lambda}\left(\bar{x}^{-1}\right)=\nu_{\Lambda}\left(x^{-1}\right)$, since $\nu_{\Lambda}$ is fixed by $\sigma$ (acting on $A$ ). Thus, if $x \in \mathfrak{K}(\Lambda) \cap G$, we have $\nu_{\Lambda}(x)=0$, whence $\mathfrak{K}(\Lambda) \cap G=P(\Lambda)$. 
Definition 1.1 ([6] (1.5), [7] (3.1)). (i) A stratum in $A$ is a 4-tuple $[\Lambda, n, r, b]$, where $\Lambda$ is an $\mathfrak{o}_{F}$-lattice sequence, $n \in \mathbb{Z}$ and $r \in \mathbb{R}$ with $n \geq r \geq 0$ and $b \in \mathfrak{a}_{-n}(\Lambda)$.

(ii) Two strata $\left[\Lambda, n, r, b_{i}\right], i=1,2$, are called equivalent if $b_{1}-b_{2} \in \mathfrak{a}_{-r}(\Lambda)$.

(iii) A stratum $[\Lambda, n, r, b]$ is called skew if $\Lambda$ is self-dual and $b \in A_{-}$.

(iv) A stratum $[\Lambda, n, r, b]$ is called null if $n=r$ and $b=0$.

Then, for $n \geq r \geq \frac{n}{2}>0$, an equivalence class of strata corresponds to a character of $U_{r+}(\Lambda)$, by

$$
[\Lambda, n, r, b] \mapsto\left(\psi_{b}: x \mapsto \psi_{A}(b(x-1)), \text { for } x \in U_{r+}\right),
$$

and an equivalence class of skew strata corresponds to a character of $P_{r+}(\Lambda)$, by

$$
[\Lambda, n, r, b] \mapsto \psi_{b}^{-}:=\left.\psi_{b}\right|_{P_{r+}} \cdot
$$

Let $[\Lambda, n, r, b]$ be a stratum in $A$. Put $y_{b}=\varpi_{F}^{n / g} b^{e / g} \in \mathfrak{a}_{0}(\Lambda)$, where $e=e(\Lambda)$ and $g=(n, e)$. Let $\Phi(X) \in \mathfrak{o}_{F}(X)$ be the characteristic polynomial of $y_{b}$. Then we define the characteristic polynomial $\varphi_{b}(X) \in k_{F}[X]$ of the stratum to be the reduction modulo $\mathfrak{p}_{F}$ of $\Phi(X)$. Note that this depends only on the equivalence class of the stratum $[\Lambda, n, n-1, b]$.

Definition 1.2. We say that the stratum $[\Lambda, n, n-1, b]$ is fundamental if $\varphi_{b}(X) \neq X^{N}$.

Now suppose that $[\Lambda, n, r, b]$ is a skew stratum in $A$. Then we have $y_{b}=\eta \bar{y}_{b}$, for $\eta= \pm$ a sign (precisely, $\eta=(-)^{e / g}$ if $F / F_{0}$ is unramified, $\eta=(-)^{n / g}(-)^{e / g}$ otherwise), and thus $\Phi(X)=\bar{\Phi}(\eta X)$ and $\varphi_{b}(X)=\bar{\varphi}_{b}(\eta X)$.

Definition 1.3. We say that the skew stratum $[\Lambda, n, n-1, b]$ is $G$-split if $\varphi_{b}(X)$ has an irreducible factor $\psi(X)$ such that $(\psi(X), \bar{\psi}(\eta X))=1$.

Definition 1.4 (6] (1.5.5). [7] (5.1)). A stratum $[\Lambda, n, r, \beta]$ in $A$ is called pure if

(i) the algebra $E=F[\beta]$ is a field;

(ii) $\Lambda$ is an $\mathfrak{o}_{E}$-lattice chain (we usually write $\Lambda_{\mathfrak{o}_{E}}$ when we are thinking of it as such);

(iii) $\nu_{\Lambda}(\beta)=-n$;

Let $[\Lambda, n, r, \beta]$ be a pure stratum and $E=F[\beta]$. We put $B=B_{\beta}=C_{A}(E)$, the $A$-centralizer of $E$, and $\mathfrak{b}_{k}=\mathfrak{a}_{k} \cap B$, for $k \in \mathbb{R}$. We also let $a_{\beta}$ denote the adjoint map (with kernel $B$ ) $x \mapsto \beta x-x \beta$, $x \in A$. For $k \in \mathbb{R}$, we put $\mathfrak{n}_{k}=\mathfrak{n}_{k}(\beta, \Lambda)=\left\{x \in \mathfrak{a}_{0}: a_{\beta}(x) \in \mathfrak{a}_{k}\right\}$. Then we define

$$
k_{0}(\beta, \Lambda)=\max \left\{-n, \max \left\{k \in \mathbb{R}: \mathfrak{n}_{k} \not \subset \mathfrak{b}_{0}+\mathfrak{a}_{1}\right\}\right\} .
$$

Note that, in the case $E=F$, this is not the same definition as in [6] (1.4.5) $\left(k_{0}(\beta, \Lambda)=-\infty\right.$ there). If $e\left(\Lambda \mid \mathfrak{o}_{E}\right)$ denotes the $\mathfrak{o}_{E}$-period of $\Lambda$, then $k_{0}(\beta, \Lambda) / e\left(\Lambda \mid \mathfrak{o}_{E}\right)$ is an integer independent of the choice of $\Lambda$; we denote it $k_{F}(\beta)$. (See [7] and [12] for more details.)

Definition 1.5 ([6] (1.5.5), [7] (5.1)). A stratum $[\Lambda, n, r, \beta]$ in $A$ is called simple if, either it is null, or it is pure and $k_{0}(\beta, \Lambda)<-r$. 
We remark that this is not quite the usual definition of simple strata since we call a null stratum simple (but see the remarks following [6] (5.5.10)). In particular, any stratum $[\Lambda, n, n, \beta]$ is equivalent to the null stratum $[\Lambda, n, n, 0]$ so we may use the null stratum as the initial step in inductive proofs "along $k_{0}(\beta, \Lambda)$ ".

Finally, for $\beta \in A$ such that $E=F[\beta]$ is a field, we put $B=C_{A}(E)$ and recall the notion of a tame corestriction $s=s_{\beta}$ on $A$ relative to $E / F([6](1.3 .3))$ : it is a $(B, B)$-bimodule homomorphism $s: A \rightarrow B$ such that $s\left(\mathfrak{a}_{0}(\Lambda)\right)=\mathfrak{b}_{0}(\Lambda)$ for all $\mathfrak{o}_{E}$-lattice sequences $\Lambda$. It is unique upto multiplication by a unit $u \in \mathfrak{o}_{E}^{\times}$and we have $s\left(\mathfrak{a}_{n}\right)=\mathfrak{b}_{n}$. If, moreover, $\beta \in A_{-}$, then there exists a tame corestriction $s$ which commutes with the involution ${ }^{-}$on $A$ (see [11 (2.1.1)); it is unique upto multiplication by a unit $u \in \mathfrak{o}_{E}^{\times}$such that $u \bar{u}=1$. Then we have $s\left(\mathfrak{a}_{n}^{-}\right)=\mathfrak{b}_{n}^{-}$.

\section{Simple characters}

In this section we recall some properties of simple characters for $\widetilde{G}$ and $G$ (see [6], [7], [13]). Many of these are only available for strict lattice sequences and we will require them for general lattice sequences, which is the main purpose of this section. We remark that, for $\widetilde{G}$, these results (and much more) have also been obtained by Secherre in [10].

We begin with a general intertwining lemma which will prove useful in extending results known for strict lattice sequences to the general case. Let $M$ be a Levi subgroup of $\widetilde{G}$ and $P_{u}$ a parabolic subgroup with Levi component $M$. Let $N_{u}$ be the unipotent radical of $P_{u}$ and let $N_{l}$ be the unipotent radical of the opposite parabolic $P_{l}$. Recall that a subgroup $H$ of $\widetilde{G}$ is said to have an Iwahori decomposition with respect to $\left(M, P_{u}\right)$ if

$$
H=\left(H \cap N_{l}\right)(H \cap M)\left(H \cap N_{u}\right) .
$$

We also recall that, given subgroups $H_{1}, H_{2}$ of $\widetilde{G}$ and representations $\rho_{1}, \rho_{2}$ of $H_{1}, H_{2}$ respectively, the intertwining in $\widetilde{G}$ of $\rho_{1}$ with $\rho_{2}$ is

$$
I_{\widetilde{G}}\left(\left.\rho_{1}\right|_{H_{1}},\left.\rho_{2}\right|_{H_{2}}\right)=\left\{g \in \widetilde{G}: \operatorname{Hom}_{{ } H_{1} \cap H_{2}}\left({ }^{g} \rho_{1}, \rho_{2}\right) \neq 0\right\},
$$

where ${ }^{g} \rho_{1}$ is the representation of ${ }^{g} H_{1}=g H_{1} g^{-1}$ given by ${ }^{g} \rho_{1}(x)=\rho_{1}\left(g^{-1} x g\right)$, for $x \in{ }^{g} H_{1}$. Notice that, if $\rho_{1}, \rho_{2}$ are characters, then $g$ intertwines $\rho_{1}$ with $\rho_{2}$ if and only if

$$
\rho_{1}\left(g^{-1} x g\right)=\rho_{2}(x), \quad \text { for all } x \in g H_{1} g^{-1} \cap H_{2} .
$$

We use analogous notation for the intertwining of representations of subgroups of $G, M$ etc.

Lemma 2.1. For $i=1,2$, let $H_{i}$ be a subgroup of $\widetilde{G}$ with an Iwahori decomposition with respect to $\left(M, P_{u}\right)$ and let $\xi_{i}$ be a character of $H_{i}$ which is trivial on $N_{l}$ and $N_{u}$. Then

$$
I_{\widetilde{G}}\left(\left.\xi_{1}\right|_{H_{1}},\left.\xi_{2}\right|_{H_{2}}\right) \cap M=I_{M}\left(\left.\xi_{1}\right|_{H_{1} \cap M},\left.\xi_{2}\right|_{H_{2} \cap M}\right) .
$$

Proof We certainly have the containment $\subset$. For the converse, we take $m \in I_{M}\left(\left.\xi_{1}\right|_{H_{1} \cap M},\left.\xi_{2}\right|_{H_{2} \cap M}\right)$ and $h_{1}=m h_{2} m^{-1} \in H_{1} \cap m H_{2} m^{-1}$; by the Iwahori decomposition, we have

$$
h_{1, l} h_{1, M} h_{1, u}=h_{1}=m h_{2} m^{-1}=\left(m h_{2, l} m^{-1}\right)\left(m h_{2, M} m^{-1}\right)\left(m h_{2, u} m^{-1}\right),
$$

where $h_{i, l} \in H_{i} \cap N_{l}, h_{i, M} \in H_{i} \cap M, h_{i, u} \in H_{i} \cap N_{u}$. But, by uniqueness of Iwahori decomposition, we have $h_{1, l}=m h_{2, l} m^{-1}$, etc.. In particular, $H_{1} \cap m H_{2} m^{-1}$ has an Iwahori decomposition. The assertion of the lemma is now trivial. 
Corollary 2.2. Let $H$ be a subgroup of $\widetilde{G}$ with an Iwahori decomposition with respect to $\left(M, P_{u}\right)$ and let $\xi$ be a character of $H$ which is trivial on $N_{l}$ and $N_{u}$. Let $m \in M$ be such that $m$ normalizes $H$. Then $m$ normalizes $\xi$ if and only if it normalizes $\left.\xi\right|_{H \cap M}$.

\subsection{Lattice sequences}

Let $[\Lambda, n, m, \beta]$ be a simple stratum in $A$. When $\Lambda$ is strict, the set of simple characters $\mathcal{C}(\Lambda, m, \beta)$ is defined in [6] (3.2) - the elements are certain arithmetically defined characters of the group $H^{m+}(\beta, \Lambda)$. (This group is defined in [6] (3.1) and denoted $H^{m+1}(\beta, \Lambda)$ there - note that, for $m$ real, we have $\mathcal{C}(\Lambda, m, \beta)=\mathcal{C}(\Lambda,\lfloor m\rfloor, \beta)$.) Moreover, a large number of properties of these characters are described in [6] $\S 3$. The definitions are extended to the case when $\Lambda$ is not strict in [7] $\S 5$ and certain of the properties are established (see also [10]). However, for our purposes, we require more of these; in particular, we calculate the intertwining.

It is convenient here to express our results in terms of ps-characters, whose definition we recall (44 §8). First, a simple pair is a pair $(k, \beta)$ consisting of a nonzero element $\beta$ generating a field extension $E$ of $F$ and a positive integer $k<k_{F}(\beta)$. Then, if we are given

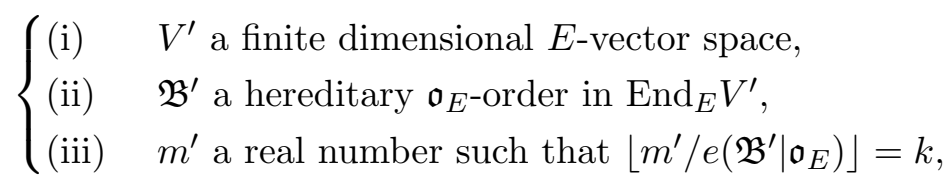

we obtain a stratum $\left[\mathfrak{A}^{\prime}, n^{\prime}, m^{\prime}, \beta\right]$ in $A^{\prime}=\operatorname{End}_{F} V^{\prime}$ as follows: $\mathfrak{A}^{\prime}$ is the hereditary $\mathfrak{o}_{F}$-order defined by the same lattice chain as $\mathfrak{B}^{\prime}$ and the integer $n^{\prime}=-\nu_{E}(\beta) e\left(\mathfrak{B}^{\prime} \mid \mathfrak{o}_{E}\right)$, where $\nu_{E}$ is the normalized valuation on $E$. The condition on $k$ means precisely that this stratum is simple, for any choice of $\left(V^{\prime}, \mathfrak{B}^{\prime}, m^{\prime}\right)$ as in $(2.3)$. Moreover, given two triples $\left(V_{i}^{\prime}, \mathfrak{B}_{i}^{\prime}, m_{i}^{\prime}\right), i=1,2$, as in (2.3), we have, by [6] (3.6.14), a canonical bijection

$$
\tau_{\mathfrak{A}_{1}^{\prime}, \mathfrak{A}_{2}^{\prime}, \beta}: \mathcal{C}\left(\mathfrak{A}_{1}^{\prime}, m_{1}^{\prime}, \beta\right) \stackrel{\sim}{\longrightarrow} \mathcal{C}\left(\mathfrak{A}_{2}^{\prime}, m_{2}^{\prime}, \beta\right) .
$$

Recall ([6] (3.6.1)) that if $V_{1}^{\prime}=V_{2}^{\prime}=V^{\prime}$ and $\theta_{1}^{\prime} \in \mathcal{C}\left(\mathfrak{A}_{1}^{\prime}, m_{1}^{\prime}, \beta\right)$ then $\tau_{\mathfrak{A}_{1}^{\prime}, \mathfrak{A}_{2}^{\prime}, \beta}\left(\theta_{1}^{\prime}\right)$ is the unique simple character $\theta_{2}^{\prime} \in \mathcal{C}\left(\mathfrak{A}_{2}^{\prime}, m_{2}^{\prime}, \beta\right)$ such that $1 \in \widetilde{G}^{\prime}$ intertwines $\theta_{1}^{\prime}$ with $\theta_{2}^{\prime}$, where $\widetilde{G}^{\prime}=\operatorname{Aut}_{F} V^{\prime}$.

A ps-character attached to a simple pair $(k, \beta)$ is a simple-character-valued function $\Theta$ which attaches to each triple $\left(V^{\prime}, \mathfrak{B}^{\prime}, m^{\prime}\right)$ as in $(2.3)$, a simple character $\Theta\left(\mathfrak{A}^{\prime}\right) \in \mathcal{C}\left(\mathfrak{A}^{\prime}, m^{\prime}, \beta\right)$ (called the realization of $\Theta$ on $\mathfrak{A}^{\prime}$ of level $m$ ) subject to the condition that, given two realizations $\Theta\left(\mathfrak{A}_{i}^{\prime}\right)$, $i=1,2$, we have $\Theta\left(\mathfrak{A}_{2}^{\prime}\right)=\tau_{\mathfrak{A}_{1}^{\prime}, \mathfrak{A}_{2}^{\prime}, \beta}\left(\Theta\left(\mathfrak{A}_{1}^{\prime}\right)\right)$. Thus a ps-character is completely determined by any one of its realizations.

Now we put ourselves in the following situation (cf. [7] (5.2)): let $[\Lambda, n, m, \beta]$ be a simple stratum in $A$, with $E=F[\beta]$ and $e=e\left(\Lambda \mid \mathfrak{o}_{E}\right)$. Let $V_{0}$ be a finite dimensional $E$-vector space and let $\Lambda^{0}$ be a strict $\mathfrak{o}_{E}$-lattice sequence in $V_{0}$ of $\mathfrak{o}_{E}$-period $e$. We put

$$
\begin{aligned}
& V^{\prime}=V \oplus V_{0} ; \\
& \Lambda^{\prime}=\Lambda \oplus \Lambda^{0} .
\end{aligned}
$$

We also put $A^{\prime}=\operatorname{End}_{F} V^{\prime}$ and $\widetilde{G}^{\prime}=\operatorname{Aut}_{F} V^{\prime}, \widetilde{G}_{0}=\operatorname{Aut}_{F} V_{0}$. Then $M=\widetilde{G}_{0} \times \widetilde{G}$ is a Levi subgroup of $\widetilde{G}^{\prime}$ and we put $N_{l}=1+\operatorname{Hom}_{F}\left(V, V_{0}\right), N_{u}=1+\operatorname{Hom}_{F}\left(V_{0}, V\right)$. We also denote by $\mathbf{1}_{V}$ the projection onto $V$ with kernel $V_{0}$. 
Now $\Lambda^{\prime}$ is a strict $\mathfrak{o}_{E}$-lattice chain in $V$ of $\mathfrak{o}_{E}$-period $e$ and $\left[\Lambda^{\prime}, n, m, \beta\right]$ is a simple stratum in $A^{\prime}$. Hence we have the set of simple characters $\mathcal{C}\left(\Lambda^{\prime}, m, \beta\right)$ of $H^{m+}\left(\beta, \Lambda^{\prime}\right)$. Then, by definition, $H^{m+}(\beta, \Lambda)=H^{m+}\left(\beta, \Lambda^{\prime}\right) \cap \widetilde{G}$ and $\mathcal{C}(\Lambda, m, \beta)$ is the set of restrictions $\left.\theta^{\prime}\right|_{H^{m+}(\beta, \Lambda)}$, for $\theta^{\prime} \in \mathcal{C}\left(\Lambda^{\prime}, m, \beta\right)$. We remark that, from [7] (5.5), this is independent of the choice of $\Lambda^{0}$; indeed it depends only on the ps-character determined by $\theta^{\prime}$, and may be thought of as the realization of this ps-character on $\Lambda$.

Let $r=-k_{0}(\beta, \Lambda)=-k_{0}\left(\beta, \Lambda^{\prime}\right)$. We put

$$
\mathfrak{M}_{m}^{\prime}=\mathfrak{M}_{m}\left(\beta, \Lambda^{\prime}\right)=\mathfrak{a}_{r-m}\left(\Lambda^{\prime}\right) \cap \mathfrak{n}_{-m}\left(\beta, \Lambda^{\prime}\right)+\mathfrak{J}^{\frac{r}{2}}\left(\beta, \Lambda^{\prime}\right),
$$

where $\mathfrak{J}^{\frac{r}{2}}\left(\beta, \Lambda^{\prime}\right)=\mathfrak{J}^{\left\lfloor\frac{r+1}{2}\right\rfloor}\left(\beta, \Lambda^{\prime}\right)$ is defined in [6] (3.1). Then, for $\theta^{\prime} \in \mathcal{C}\left(\Lambda^{\prime}, m, \beta\right)$, we have

$$
I_{\widetilde{G}^{\prime}}\left(\theta^{\prime} \mid H^{m+}\left(\beta, \Lambda^{\prime}\right)\right)=\left(1+\mathfrak{M}_{m}^{\prime}\right) B^{\prime \times}\left(1+\mathfrak{M}_{m}^{\prime}\right),
$$

where $B^{\prime}=\operatorname{End}_{E} V^{\prime}$, by [6] (3.3.2).

Proposition 2.5. Let $\theta \in \mathcal{C}(\Lambda, m, \beta)$ so that $\theta=\left.\theta^{\prime}\right|_{H^{m+}(\beta, \Lambda)}$, for some $\theta^{\prime} \in \mathcal{C}\left(\Lambda^{\prime}, m, \beta\right)$. Then

$$
I_{\widetilde{G}}\left(\theta \mid H^{m+}(\beta, \Lambda)\right)=\left(1+\mathfrak{M}_{m}\right) B^{\times}\left(1+\mathfrak{M}_{m}\right),
$$

where $\mathfrak{M}_{m}=\mathfrak{M}_{m}(\beta, \Lambda)=\mathfrak{M}_{m}^{\prime} \cap A=\mathbf{1}_{V} \mathfrak{M}_{m}^{\prime} \mathbf{1}_{V}$ and $B=\operatorname{End}_{E} V$.

Proof We remark first that, by [7] (5.6), $\mathfrak{M}_{m}$ is independent of the choice of $\Lambda^{0}$. We abbreviate $H^{\prime}=H^{m+}\left(\beta, \Lambda^{\prime}\right)$ and $H=H^{m+}(\beta, \Lambda)$.

By Lemma 2.1, we have $I_{M}\left(\theta^{\prime} \mid H^{\prime} \cap M\right)=I_{M}\left(\theta^{\prime} \mid H^{\prime}\right)=\left(1+\mathfrak{M}_{m}^{\prime}\right) B^{\prime \times}\left(1+\mathfrak{M}_{m}^{\prime}\right) \cap M$ and this is precisely $\left(1+\mathfrak{M}_{m}^{\prime}\right) \cap M \cdot B^{\prime} \cap M \cdot\left(1+\mathfrak{M}_{m}^{\prime}\right) \cap M$ by [12] (1.3) (cf. op. cit. (3.15)). But then

$$
\begin{aligned}
I_{\widetilde{G}}(\theta \mid H) & =I_{\widetilde{G}}\left(\theta^{\prime} \mid H^{\prime} \cap M\right) \\
& =\left(\left(1+\mathfrak{M}_{m}^{\prime}\right) \cap M \cdot B^{\prime} \cap M \cdot\left(1+\mathfrak{M}_{m}^{\prime}\right) \cap M\right) \cap \widetilde{G} \\
& =\left(1+\mathfrak{M}_{m}\right) B^{\times}\left(1+\mathfrak{M}_{m}\right)
\end{aligned}
$$

as required.

Lemma 2.6. Let $\Theta$ be a ps-character attached to the simple pair $(k, \beta)$. For $i=1,2$, let $\Lambda^{i}$ be an $\mathfrak{o}_{E}$-lattice sequence of $\mathfrak{o}_{E}$-period $e$ in a finite dimensional E-vector space $V$ and let $m_{i} \in \mathbb{R}$ be such that $\left\lfloor m_{i} / e\right\rfloor=k$. Let $\theta_{i}=\Theta\left(\Lambda^{i}\right)$ be the realization of $\Theta$ on $\Lambda^{i}$ of level $m_{i}$. Then we have

$$
1 \in I_{\widetilde{G}}\left(\theta_{1}, \theta_{2}\right) .
$$

Moreover, $\theta_{2}$ is the unique simple character in $\mathcal{C}\left(\Lambda^{2}, m_{2}, \beta\right)$ such that $1 \in I_{\widetilde{G}}\left(\theta_{1}, \theta_{2}\right)$.

Proof As above, let $V_{0}$ be a finite dimensional $E$-vector space and let $\Lambda^{0}$ be a strict $\mathfrak{o}_{E}$-lattice sequence in $V_{0}$ of $\mathfrak{o}_{E}$-period $e$. We put $V^{\prime}=V \oplus V_{0}, \Lambda_{1}^{\prime}=\Lambda^{1} \oplus \Lambda^{0}, \Lambda_{2}^{\prime}=\Lambda^{2} \oplus \Lambda^{0}$ and also $A^{\prime}=\operatorname{End}_{F} V^{\prime}$ and $\widetilde{G}^{\prime}=\operatorname{Aut}_{F} V^{\prime}$.

For $i=1,2$, let $\theta_{i}^{\prime}=\Theta\left(\Lambda_{i}^{\prime}\right)$ be the realization of $\Theta$ on the strict lattice sequence $\Lambda_{i}^{\prime}$ of level $m_{i}$, so we have $H^{m_{i}+}\left(\beta, \Lambda_{i}^{\prime}\right) \cap \widetilde{G}=H^{m_{i}+}\left(\beta, \Lambda^{i}\right)$ and $\left.\theta_{i}^{\prime}\right|_{H^{m_{i}+}\left(\beta, \Lambda^{i}\right)}=\theta_{i}$. Then the first assertion follows immediately from Lemma 2.1 .

For the final assertion, suppose $\theta_{2}^{\prime}=\Theta^{\prime}\left(\Lambda^{2}\right)$ and $1 \in I_{\widetilde{G}}\left(\theta_{1}, \theta_{2}^{\prime}\right)$. Consider $V_{0}=V \oplus \cdots \oplus V(e$ times) and, for $i=1,2$, the strict lattice sequence $\Lambda_{0}^{i}$ given by

$$
\Lambda_{0}^{i}(k)=\Lambda^{i}(k) \oplus \Lambda^{i}(k+1) \oplus \cdots \oplus \Lambda^{i}(k+e-1), \quad \text { for } k \in \mathbb{Z} .
$$


Put $\theta_{0}=\Theta\left(\Lambda_{0}^{1}\right)$ and $\theta_{0}^{\prime}=\Theta^{\prime}\left(\Lambda_{0}^{2}\right)$. Writing $M=\widetilde{G} \times \cdots \times \widetilde{G} \subset \widetilde{G}_{0}=$ Aut $_{F} V_{0}$, we have $\left.\theta_{0}\right|_{M}=$ $\theta_{1} \otimes \cdots \otimes \theta_{1}$ and $\left.\theta_{0}^{\prime}\right|_{M}=\theta_{2}^{\prime} \otimes \cdots \otimes \theta_{2}^{\prime}$; in particular, $1 \in I_{M}\left(\left.\theta_{0}\right|_{M},\left.\theta_{0}^{\prime}\right|_{M}\right)$. But, by [7] (5.2) Proposition, $\theta_{0}, \theta_{0}^{\prime}$ restrict trivially to the unipotent radical of any parabolic subgroup with Levi factor $M$ so, by Lemma 2.1, we have $1 \in I_{\widetilde{G}_{0}}\left(\theta_{0}, \theta_{0}^{\prime}\right)$. But each $\Lambda_{0}^{i}$ is strict so, by [6] (3.6.1), the characters $\theta_{0}$, $\theta_{0}^{\prime}$ correspond under the canonical bijection $\tau_{\Lambda_{0}^{1}, \Lambda_{0}^{2}, \beta}$ in 2.4 . Hence $\theta_{0}^{\prime}=\Theta\left(\Lambda_{0}^{2}\right)$ also and, since a ps-character is determined by one of its realizations, we have $\Theta=\Theta^{\prime}$, as required.

We will sometimes use $\tau_{\Lambda^{1}, \Lambda^{2}, \beta}$ to denote the correspondence $\mathcal{C}\left(\Lambda^{1}, m_{1}, \beta\right) \stackrel{\sim}{\longrightarrow} \mathcal{C}\left(\Lambda^{2}, m_{2}, \beta\right)$ given by the ps-characters.

Recall that, if we have $[\Lambda, n, r, \beta]$ pure, with $\Lambda$ strict and $r=-k_{0}(\beta, \Lambda)$, and $[\Lambda, n, r, \gamma]$ is simple and equivalent to $[\Lambda, n, r, \beta]$, then the map

$$
\begin{aligned}
\mathcal{C}(\Lambda, m, \beta) & \rightarrow \mathcal{C}(\Lambda, m, \gamma) \\
\theta & \mapsto \theta \psi_{\gamma-\beta}
\end{aligned}
$$

is bijective for $\frac{r}{2} \leq m<r$, by [6] (3.3.18). Note that this then clearly holds also when $\Lambda$ is not necessarily strict.

In fact, there will be several occasions when we will need to be careful in the way we choose a $\gamma$ as above. To describe this, we recall the notion of a "generalized $(W, E)$-decomposition" from [7] $\S 5.3$ (see also [6] $\S 1.2$ ).

Let $E / F$ be a field extension in $A$ and put $B=\operatorname{End}_{E} V$. We write $A(E)=\operatorname{End}_{F} E$ and $\mathfrak{A}(E)$ for the unique hereditary order in $A(E)$ normalized by $E^{\times}$. Let $W$ be the $F$-span of an $E$-basis of $V$. Then the isomorphism $E \otimes_{F} W \rightarrow V$ induces an isomorphism of $(A(E), B)$-bimodules.

$$
A(E) \otimes_{E} B \simeq A .
$$

In particular, the choice of $W$ also induces an embedding of algebras $\iota_{W}: A(E) \hookrightarrow A$ extending the embedding of $E$ in $A$.

Now let $\Lambda$ be an $\mathfrak{o}_{E}$-lattice sequence in $V$, which we may also view as an $\mathfrak{o}_{F}$-lattice sequence. We put $\mathfrak{b}_{n}(\Lambda)=\mathfrak{a}_{n}(\Lambda) \cap B$, for $n \in \mathbb{R}$. We say that $W$ is in general position relative to $\Lambda$ over $E$ if $W$ has an $F$ basis $w_{1}, \ldots, w_{m}$ such that, for each $k \in \mathbb{Z}$, there are integers $k(i), 1 \leq i \leq m$, such that

$$
\Lambda(k)=\mathfrak{p}_{E}^{k(1)} w_{1} \oplus \cdots \oplus \mathfrak{p}_{E}^{k(m)} w_{m} .
$$

(That is, $W$ is the $F$-span of an $E$-basis of $V$ which is a splitting of $\Lambda$ - see \$3.1.) Then [7] 5.3 Lemma says that, for such $W$, we have isomorphisms

$$
\mathfrak{A}(E) \otimes_{\mathfrak{o}_{E}} \mathfrak{b}_{n}(\Lambda) \simeq \mathfrak{a}_{n}(\Lambda), \quad n \in \mathbb{R},
$$

of $\left(\mathfrak{A}(E), \mathfrak{b}_{0}(\Lambda)\right)$-bimodules.

Now suppose $[\Lambda, n, r, \beta]$ is a pure stratum with $r=-k_{0}(\beta, \Lambda)$ and $E=F[\beta]$. Then [7] 5.3 Corollary states that, for $W$ in general position relative to $\Lambda$ over $E_{\beta},[\Lambda, n, r, \beta]$ is equivalent to a simple stratum $[\Lambda, n, r, \gamma]$ with $\gamma \in \iota_{W}(\mathfrak{K}(\mathfrak{A}(E)))$.

Now, for $i=1,2$, let $\left[\Lambda^{i}, n, r, \beta\right]$ be a pure stratum in $A^{i}=\operatorname{End}_{F} V_{i}$, with $e=e\left(\Lambda^{i} \mid \mathfrak{o}_{E_{\beta}}\right)$ and $r=-k_{0}\left(\beta, \Lambda^{i}\right)$. Put $V=V_{1} \oplus V_{2}, A=\operatorname{End}_{F} V$ and $\Lambda=\Lambda^{1} \oplus \Lambda^{2}$; then $[\Lambda, n, r, \beta]$ is a pure stratum in $A$. For $i=1,2$, let $W_{i}$ be in general position relative to $\Lambda^{i}$ over $E_{\beta}$ and put $W=W_{1} \oplus W_{2}$, 
which is in general position relative to $\Lambda$ over $E_{\beta}$. Then there exists $\gamma \in \iota_{W}(\mathfrak{K}(\mathfrak{A}(E))) \subset A^{1} \oplus A^{2}$ such that $[\Lambda, n, r, \gamma]$ is simple and equivalent to $[\Lambda, n, r, \beta]$. In particular, we can regard $\gamma$ as an element of $A, A^{1}$ or $A^{2}$ and, for $i=1,2,\left[\Lambda^{i}, n, r, \gamma\right]$ is a simple stratum equivalent to $\left[\Lambda^{i}, n, r, \beta\right]$. Then, for $\theta \in \mathcal{C}(\Lambda, m, \beta)$ with $\frac{r}{2} \leq m<r$ and $i=1,2$, we have

$$
\tau_{\Lambda, \Lambda^{i}, \beta}(\theta) \psi_{\gamma-\beta}=\tau_{\Lambda, \Lambda^{i}, \gamma}\left(\theta \psi_{\gamma-\beta}\right),
$$

since the transfer maps are simply restriction. In particular, we obtain that, for $\theta \in \mathcal{C}\left(\Lambda^{1}, m, \beta\right)$, we have $\tau_{\Lambda^{1}, \Lambda^{2}, \beta}(\theta) \psi_{\gamma-\beta}=\tau_{\Lambda^{1}, \Lambda^{2}, \gamma}\left(\theta \psi_{\gamma-\beta}\right)$.

\subsection{The orders $\mathfrak{H}$ and $\mathfrak{J}$}

Let $[\Lambda, n, 0, \beta]$ be a simple stratum in $A$. If $\Lambda$ is strict then the orders $\mathfrak{H}(\beta, \Lambda)$ and $\mathfrak{J}(\beta, \Lambda)$ are defined in [6] (3.1); if $\Lambda$ is not strict, they are defined in [7] by restriction from a larger space, as in the previous section. However, it would be possible to make the definitions directly as in 6] (3.1). In this section we show that these two definitions coincide. In fact, this will follow almost immediately from the following two lemmas. We suppose $V=V_{1} \oplus V_{2}$ and use our standard block notation.

Lemma 2.8. Let $X, Y$ be $\mathfrak{o}_{F}$-lattices in $A$ such that $\mathbf{1}^{i} X \mathbf{1}^{j} \subset X$ and $\mathbf{1}^{i} Y \mathbf{1}^{j} \subset Y$, for $i, j=1,2$. Then, for $i, j=1,2$,

(i) $\mathbf{1}^{i}(X+Y) \mathbf{1}^{j}=\mathbf{1}^{i} X \mathbf{1}^{j}+\mathbf{1}^{i} Y \mathbf{1}^{j}$;

(ii) $\mathbf{1}^{i}(X \cap Y) \mathbf{1}^{j}=\mathbf{1}^{i} X \mathbf{1}^{j} \cap \mathbf{1}^{i} Y \mathbf{1}^{j}$.

Proof (i) is clear while for (ii) we have $\mathbf{1}^{i}(X \cap Y) \mathbf{1}^{j} \subset \mathbf{1}^{i} X \mathbf{1}^{j} \cap \mathbf{1}^{i} Y \mathbf{1}^{j} \subset X \cap Y$; then, applying $\mathbf{1}^{i}$ on the left and $\mathbf{1}^{j}$ on the right, we have the required equality.

Lemma 2.9. Let $X$ be as in the previous lemma. Define $\left(\mathbf{1}^{i} X \mathbf{1}^{i}\right)^{*}=\left\{a \in A^{i}: \operatorname{tr}_{A^{i} / F}\left(a \mathbf{1}^{i} X \mathbf{1}^{i}\right) \subset\right.$ $\left.\mathfrak{p}_{F}\right\}$. Then

$$
\left(\mathbf{1}^{i} X \mathbf{1}^{i}\right)^{*}=\mathbf{1}^{i} X^{*} \mathbf{1}^{i}
$$

Proof Straightforward properties of trace.

Let now $[\Lambda, n, 0, \beta]$ be a simple stratum in $A$ with $r=-k_{0}(\beta, \Lambda)$ and $e=e\left(\Lambda \mid \mathfrak{o}_{E}\right)$, where $E=F[\beta]$. Let $V_{0}$ be a finite dimensional $E$-vector space and let $\Lambda^{0}$ be a strict regular $\mathfrak{o}_{E}$-lattice sequence in $V_{0}$ of $\mathfrak{o}_{E}$-period $e$, where regular means that $\operatorname{dim}_{k_{E}} \Lambda^{0}(i) / \Lambda^{0}(i+1)$ is independent of $i$ (so that the associated hereditary order $\mathfrak{b}_{\beta, 0}=\mathfrak{a}_{0} \cap B_{\beta}$ is principal, where $B_{\beta}$ is the centralizer of $\beta$ ). Note that, since $\Lambda^{0}$ is regular, the valuation map $\nu_{\Lambda^{0}}: \mathfrak{K}\left(\Lambda_{\mathfrak{o}_{E}}^{0}\right) \rightarrow \mathbb{Z}$ is surjective.

We put $V^{\prime}=V \oplus V_{0}, \Lambda^{\prime}=\Lambda \oplus \Lambda^{0}$. Using a generalized ( $\left.W, E\right)$-decomposition as above, we choose $\gamma$ such that $[\Lambda, n, r, \gamma]$ is a simple stratum equivalent to $[\Lambda, n, r, \beta]$ and $\left[\Lambda^{\prime}, n, r, \gamma\right]$ is simple and equivalent to $\left[\Lambda^{\prime}, n, r, \beta\right]$. Now Lemma 2.8 implies immediately that we have $\mathfrak{H}(\beta, \Lambda)=\mathfrak{b}_{\beta, 0}+$ $\mathfrak{H}^{\frac{r}{2}+}(\gamma, \Lambda)$ and $\mathfrak{J}(\beta, \Lambda)=\mathfrak{b}_{\beta, 0}+\mathfrak{J}^{\frac{r}{2}}(\gamma, \Lambda)$. 
We also observe that, since $\Lambda^{0}$ is regular, we have $\mathfrak{K}\left(\Lambda_{\mathfrak{o}_{E}}\right)=\mathbf{1}_{V} \mathfrak{K}\left(\Lambda_{\mathfrak{o}_{E}}^{\prime}\right) \mathbf{1}_{V}$ : the containment $\supset$ is clear; conversely, for $x \in \mathfrak{K}\left(\Lambda_{\mathfrak{o}_{E}}\right)$, there exists $x_{0} \in \mathfrak{K}\left(\Lambda_{\mathfrak{o}_{E}}^{0}\right)$ such that $\nu_{\Lambda}(x)=\nu_{\Lambda^{0}}\left(x_{0}\right)$ and then

$$
x=\mathbf{1}_{V}\left(\begin{array}{cc}
x & 0 \\
0 & x_{0}
\end{array}\right) \mathbf{1}_{V} \in \mathbf{1}_{V} \mathfrak{K}\left(\Lambda_{\mathfrak{o}_{E}}^{\prime}\right) \mathbf{1}_{V}
$$

Now the main results of [6] (3.1) follow easily for an arbitrary lattice sequence. In particular we have

Lemma 2.10 (cf. [6] (3.1.9-13)). (i) For $0 \leq t \leq r, \mathfrak{H}^{\frac{t}{2}+}(\beta, \Lambda)$ is a bimodule over the ring $\mathfrak{n}_{-t}(\beta, \Lambda)$, as is $\mathfrak{J}^{\frac{t}{2}}(\beta, \Lambda)$.

(ii) For $k \in \mathbb{R}, \mathfrak{H}^{k}(\beta, \Lambda) \subset \mathfrak{J}^{k}(\beta, \Lambda)$ are invariant under conjugation by $\mathfrak{K}\left(\Lambda_{\mathfrak{o}_{E}}\right)$.

(iii) For $k, l>0, \mathfrak{J}^{k}(\beta, \Lambda) \mathfrak{J}^{l}(\beta, \Lambda) \subset \mathfrak{H}^{k+l}(\beta, \Lambda)$.

(iv) For $k>0, \mathfrak{H}^{k}(\beta, \Lambda)$ is a two-sided ideal of $\mathfrak{J}(\beta, \Lambda)$.

\subsection{Simple characters for $G$}

We say that a simple pair $(k, \beta)$ is skew if the galois involution ${ }^{-}$can be extended to $E=F[\beta]$ in such a way that $\bar{\beta}=-\beta$. Then, if we are given

$$
\left\{\begin{array}{l}
\text { (i) } \quad V^{\prime} \text { a finite dimensional } E \text {-vector space equipped with an } \epsilon \text {-hermitian } \\
\text { form } f^{\prime}: V^{\prime} \times V^{\prime} \rightarrow E, \\
\text { (ii) } \quad \mathfrak{B}^{\prime} \text { a hereditary } \mathfrak{o}_{E} \text {-order in } \operatorname{End}_{E} V^{\prime} \text { fixed by the involution }{ }^{-} \\
\text {induced by } f^{\prime}, \\
\text { (iii) } \quad m^{\prime} \text { a real number such that }\left\lfloor m^{\prime} / e\left(\mathfrak{B}^{\prime} \mid \mathfrak{o}_{E}\right)\right\rfloor=k,
\end{array}\right.
$$

we obtain a skew stratum $\left[\mathfrak{A}^{\prime}, n^{\prime}, m^{\prime}, \beta\right]$ in $A^{\prime}=\operatorname{End}_{F} V^{\prime}$ as follows: we choose an $F_{0}$-linear form $\lambda_{0}$ on $E_{0}$ such that

$$
\left\{e \in E_{0} ; \lambda_{0}\left(e \mathfrak{o}_{E_{0}}\right) \subset \mathfrak{p}_{F_{0}}\right\}=\mathfrak{p}_{E_{0}}
$$

(as in [3] $\S 5$ ), and let $\lambda$ be the $F$-linear form on $E$ given either by extending linearly (if $F \neq F_{0}$ ) or by composing with $\operatorname{tr}_{E / E_{0}}$ (if $F=F_{0}$ ); then $V^{\prime}$, as an $F$-vector space, is equipped with the $\epsilon$-hermitian form $h^{\prime}=\lambda \circ f^{\prime}$. Note that the duality induced by $h^{\prime}$ is independent of the choice of $\lambda_{0}$.

We say that a ps-character $\Theta$ attached to a skew simple pair $(k, \beta)$ is self-dual if there exists a triple as in 2.11 such that the realization $\Theta\left(\mathfrak{A}^{\prime}\right) \in \mathcal{C}\left(\mathfrak{A}^{\prime}, m^{\prime}, \beta\right)$ is fixed by $\sigma: x \mapsto \bar{x}^{-1}, x \in \widetilde{G}^{\prime}$, where $\widetilde{G}^{\prime}=\operatorname{Aut}_{F} V^{\prime}$.

Proposition 2.12. Let $(k, \beta)$ be a skew simple pair and, for $i=1,2$, let $V_{i}^{\prime}$ be an E-vector space as in (2.11) (i), $\Lambda_{i}^{\prime}$ be a self-dual $\mathfrak{o}_{E}$-lattice sequence of $\mathfrak{o}_{E}$-period $e_{i}^{\prime}$ in $V_{i}^{\prime}$ and $m_{i}^{\prime} \in \mathbb{R}$ be such that $\left\lfloor m_{i}^{\prime} / e_{i}^{\prime}\right\rfloor=k$. Then the canonical bijection $\tau_{\Lambda_{1}^{\prime}, \Lambda_{2}^{\prime}, \beta}$ commutes with $\sigma$.

Proof Let $\Lambda_{0}^{\prime}$ be a strict self-dual $\mathfrak{o}_{E}$-lattice sequence of $\mathfrak{o}_{E}$-period $e_{0}^{\prime}=l \mathrm{~cm}\left(e_{1}^{\prime}, e_{2}^{\prime}\right)$ in an $E$-vector space $V_{0}^{\prime}$ as in (2.11) (i) and let $m_{0}^{\prime}=k e_{0}^{\prime}$. We consider the $E$-vector space $V^{\prime}=V_{0}^{\prime} \perp V_{1}^{\prime} \perp V_{2}^{\prime}$ equipped with the form $f^{\prime}=f_{0}^{\prime} \perp f_{1}^{\prime} \perp f_{2}^{\prime}$, that is

$$
f^{\prime}\left(v_{0}+v_{1}+v_{2}, w_{0}+w_{1}+w_{2}\right)=f_{0}^{\prime}\left(v_{0}, w_{0}\right)+f_{1}^{\prime}\left(v_{1}, w_{1}\right)+f_{2}^{\prime}\left(v_{2}, w_{2}\right), \quad \text { for } v_{i}, w_{i} \in V_{i}^{\prime} .
$$


Set $\Lambda=\Lambda_{0}^{\prime} \perp \Lambda_{1}^{\prime} \perp \Lambda_{2}^{\prime}$, a strict self-dual $\mathfrak{o}_{E}$-lattice sequence of $\mathfrak{o}_{E}$-period $e^{\prime}=e_{0}^{\prime}$ in $V^{\prime}$, and $m^{\prime}=m_{0}^{\prime}$. Then we have $\tau_{\Lambda_{1}^{\prime}, \Lambda_{2}^{\prime}, \beta}=\tau_{\Lambda^{\prime}, \Lambda_{2}^{\prime}, \beta} \circ \tau_{\Lambda^{\prime}, \Lambda_{1}^{\prime}, \beta}^{-1}$ so we need only check that $\tau_{\Lambda^{\prime}, \Lambda_{1}^{\prime}, \beta}$ commutes with $\sigma$, by symmetry.

Let $\theta \in \mathcal{C}\left(\Lambda^{\prime}, m^{\prime}, \beta\right), \theta_{1} \in \mathcal{C}\left(\Lambda_{1}^{\prime}, m_{1}^{\prime}, \beta\right)$ be such that $\theta_{1}=\tau_{\Lambda^{\prime}, \Lambda_{1}^{\prime}, \beta}(\theta)$. Then, by the definition of $\mathcal{C}\left(\Lambda_{1}^{\prime}, m_{1}^{\prime}, \beta\right)$ (cf. [7] (5.5)), we have $\theta_{1}=\left.\theta\right|_{\widetilde{G}_{1}}$, where $\widetilde{G}_{1}=\operatorname{Aut}_{F} V_{1}^{\prime}$. But then $\theta_{1}^{\sigma}=\left.\theta^{\sigma}\right|_{\widetilde{G}_{1}}$ so, again by definition, $\theta_{1}^{\sigma}=\tau_{\Lambda^{\prime}, \Lambda_{1}^{\prime}, \beta}\left(\theta^{\sigma}\right)$ as required.

Corollary 2.13. Let $\Theta$ be a self-dual ps-character attached to the skew simple pair $(k, \beta)$. Let $V$ be an E-vector space as in 2.11)(i), $\Lambda$ be a self-dual $\mathfrak{o}_{E}$-lattice sequence of $\mathfrak{o}_{E}$-period $e$ in $V$ and $m \in \mathbb{R}$ be such that $\lfloor m / e\rfloor=k$. Then the realization $\theta=\Theta(\Lambda)$ on $\Lambda$ of level $m$ is fixed by $\sigma$.

In particular, a ps-character is self-dual if and only if every realization of it is fixed by $\sigma$.

\section{Semisimple characters}

In [13, the author defined semisimple characters for split semisimple strata. Here we extend this definition to the "relatively split" case of [7] $\S 6$. We lay down the groundwork in $\$ 3.1$ and define the relevant groups and semisimple characters in $\$ 3.2$. The main results are then the calculation of the intertwining of semisimple character (Theorem 3.22) and the transfer property (Proposition 3.26). In $\S 3.6$, we let the involution $\sigma$ act and obtain all the analogous results for our classical group $G$.

\subsection{Preparation}

Let $[\Lambda, n, r, \beta]$ be a stratum in $A$ and suppose we have a decomposition $V=\bigoplus_{i=1}^{l} V^{i}$. Let $\Lambda^{i}$ be the lattice sequence in $V^{i}$ given by $\Lambda^{i}(k)=\Lambda(k) \cap V^{i}$ and put $\beta_{i}=\mathbf{1}^{i} \beta \mathbf{1}^{i}$, where $\mathbf{1}^{i}$ is the projection onto $V^{i}$ with kernel $\bigoplus_{j \neq i} V^{j}$. We say that $V=\bigoplus_{i=1}^{l} V^{i}$ is a splitting for the stratum $[\Lambda, n, r, \beta]$ if we have $\Lambda(k)=\bigoplus_{i=1}^{l} \Lambda^{i}(k)$, for all $k \in \mathbb{Z}$, and $\beta=\sum_{i=1}^{l} \beta_{i}$. Similarly, we say that a basis $v_{1}, \ldots, v_{N}$ for $V$ is a splitting of $\Lambda$ (respectively the stratum) if $V=\bigoplus_{i=1}^{N} F v_{i}$ is a splitting for it.

Whenever we have such a splitting, we will use the block notation $A^{i j}=\operatorname{Hom}_{F}\left(V^{j}, V^{i}\right)$ as in $\$ 1.1$ In particular, $\mathcal{M}=\bigoplus_{i=1}^{l} A^{i i}$

Definition 3.1 (cf. [7] (3.6)). A stratum $[\Lambda, n, n-1, \beta]$ in $A$ is called split if there exists a splitting $V=\bigoplus_{i=1}^{l} V^{i}$ such that the characteristic polynomials $\phi_{i}(X)$ of $\left[\Lambda^{i}, n, n-1, \beta_{i}\right], i=1, \ldots, l$ are pairwise coprime.

Definition 3.2. A stratum $[\Lambda, n, r-, \beta]$ in $A$ is called semisimple if either it is null or $\nu_{\Lambda}(\beta)=-n$ and there exists a splitting $V=\bigoplus_{i=1}^{l} V^{i}$ for the stratum such that

(i) for $1 \leq i \leq l,\left[\Lambda^{i}, q_{i}, r-, \beta_{i}\right]$ is a simple or null stratum, where $q_{i}=r-$ if $\beta_{i}=0, q_{i}=-\nu_{\Lambda^{i}}\left(\beta_{i}\right)$ otherwise;

(ii) for $1 \leq i, j \leq l, i \neq j,\left[\Lambda^{i} \oplus \Lambda^{j}, q, r-, \beta_{i}+\beta_{j}\right]$ is not equivalent to a simple or null stratum, with $q=\max \left\{q_{i}, q_{j}\right\}$.

Remarks 3.3. (i) A simple stratum is semisimple, with the trivial splitting. We will also consider null strata $[\Lambda, n, n, 0]$ as a special case of simple strata, as in $\$ 1.2$. 
(ii) A non-simple semisimple stratum $[\Lambda, n, n-1, \beta]$ is certainly split (by a coarsening of the same splitting), by [6] (2.5.8).

(iii) If $[\Lambda, n, r-, \beta]$ is a semisimple stratum then the associated splitting $V=\bigoplus_{i=1}^{l} V^{i}$ is determined (upto order) by $\beta$ : for $i=1, . ., l$, let $\Psi_{i}(X)$ denote the minimal polynomial of $\beta_{i}$, which is irreducible since $F\left[\beta_{i}\right]$ is a field; then the $\Psi_{i}(X)$ are distinct (so pairwise coprime), by condition (ii) of Definition 3.2 so the minimal polynomial of $\beta$ is $\prod_{i=1}^{l} \Psi_{i}(X)$ and $V^{i}=\operatorname{ker} \Psi_{i}(\beta)$.

Note that any stratum satisfying condition (i) of Definition 3.2 is clearly equivalent to a semisimple stratum, by coarsening the splitting suitably. In particular, for $1 \leq i \leq l$, let $\left[\Lambda^{i}, q_{i}, r, \gamma_{i}^{\prime}\right]$ be a simple or null stratum equivalent to $\left[\Lambda^{i}, q_{i}, r, \beta_{i}\right]$ and put $\gamma^{\prime}=\sum_{i=1}^{l} \gamma_{i}^{\prime}$. Then $\left[\Lambda, n, r, \gamma^{\prime}\right]$ satisfies (i) and hence is equivalent to a semisimple stratum $[\Lambda, n, r, \gamma]$, with splitting $V=\bigoplus_{j=1}^{m} V^{I_{j}}$, where $\{1, \ldots, l\}=\bigcup_{j=1}^{m} I_{j}$ and $V^{I_{j}}=\bigoplus_{i \in I_{j}} V^{i}$. This allows us to proceed by induction along $r$ for semisimple strata. (Note that, although we allow real values of $r$, only integer values really play a role.)

In fact, we will have to be a little more careful in the way in which we choose $\gamma$. Recall that, given $[\Lambda, n, 0, \beta]$ a simple stratum with $r=-k_{0}(\beta, \Lambda)$ and $E=F[\beta]$, 7] 5.3 Corollary states that, for $W$ in general position relative to $\Lambda$ over $E,[\Lambda, n, r, \beta]$ is equivalent to a simple stratum $[\Lambda, n, r, \gamma]$ with $\gamma \in \iota_{W}(\mathfrak{K}(\mathfrak{A}(E)))$. We show now that we have a similar result for semisimple strata.

Proposition 3.4. Let $[\Lambda, n, r-, \beta]$ be a semisimple stratum in $A$, split by $V=\oplus_{i=1}^{l} V^{i}$ and such that $[\Lambda, n, r, \beta]$ is equivalent to a simple stratum $[\Lambda, n, r, \gamma]$. Put $E_{i}=F\left[\beta_{i}\right]$; then, given $W^{i}$ in general position relative to $\Lambda^{i}$ over $E_{i}$, for $1 \leq i \leq l$, we may choose $\gamma \in \prod \iota_{W^{i}}\left(\mathfrak{K}\left(\mathfrak{A}\left(E_{i}\right)\right)\right) \subset \mathcal{M}$.

Proof We show, by induction, that $[\Lambda, n, t, \beta]$ is equivalent to a simple stratum $\left[\Lambda, n, t, \gamma^{(t)}\right]$ as required, $n-1 \geq t \geq r, t \in \mathbb{Z}$.

Let $\psi_{i}(X)=\varphi_{i}(X)^{d_{i}}$ be the characteristic polynomial of the stratum $\left[\mathfrak{A}\left(E_{i}\right), n_{i}, n_{i}-1, \beta_{i}\right]$, where $n_{i}=n / e\left(\Lambda^{i} \mid \mathfrak{o}_{E_{i}}\right)$. Then the characteristic polynomial of $\left[\Lambda^{i}, n, n-1, \beta_{i}\right]$ is $\psi_{i}(X)^{\delta_{i}}$, where $\delta_{i}=$ $\operatorname{dim}_{E_{i}} V^{i}$, so the characteristic polynomial of $[\Lambda, n, n-1, \beta]$ is $\prod_{i=1}^{l} \psi_{i}(X)^{\delta_{i}}$. This stratum is equivalent to a simple stratum so it is non-split; hence $\varphi_{i}(X)=\varphi(X)$, for $1 \leq i \leq l$.

Now we choose $\Phi(X) \in \mathfrak{o}_{F}[X]$ such that $\varphi(X)=\Phi(X)\left(\bmod \mathfrak{p}_{F}\right)$. Then, by [6] (2.5.11), we can find simple strata $\left[\mathfrak{A}\left(E_{i}\right), n_{i}, n_{i}-1, \gamma_{i}\right]$ equivalent to $\left[\mathfrak{A}\left(E_{i}\right), n_{i}, n_{i}-1, \beta_{i}\right]$ such that the minimal polynomial of $\gamma_{i}$ is $\Phi\left(\left(\varpi_{F}^{-n / g} X\right)^{e / g}\right)$. Then we put $\gamma^{(n-1)}=\sum_{i=1}^{l} \iota_{W^{i}}\left(\gamma_{i}\right)$.

Now suppose we have found $\left[\Lambda, n, t, \gamma^{(t)}\right]$ equivalent to $[\Lambda, n, t, \beta]$ as required. We will omit the superscript $^{(t)}$ and put $E_{\gamma}=F[\gamma]$. We choose tame corestrictions $s_{\gamma}$ on $A, A\left(E_{i}\right)$ which are compatible with the (W,E)-decompositions (cf. [6] (1.3.9), (2.2.8)).

We have $\left[\mathfrak{A}\left(E_{i}\right), n_{i}, t_{i}, \gamma\right] \sim\left[\mathfrak{A}\left(E_{i}\right), n_{i}, t_{i}, \beta_{i}\right]$, with $e_{i}=e\left(\Lambda^{i} \mid \mathfrak{o}_{E_{i}}\right), t_{i}=\left\lfloor\frac{t}{e_{i}}\right\rfloor$. Let $\left[\mathfrak{A}\left(E_{i}\right), n_{i},\left\lfloor\frac{t-1}{e_{i}}\right\rfloor, \xi_{i}\right]$

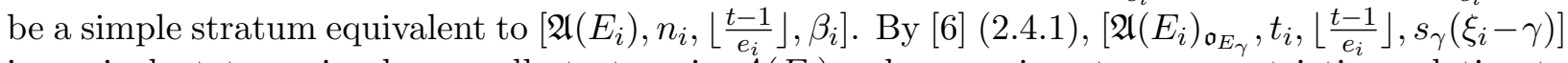
is equivalent to a simple or null stratum in $A\left(E_{i}\right)$, where $s_{\gamma}$ is a tame corestriction relative to $E_{\gamma} / F$. Let $\psi_{i}(X)=\varphi_{i}(X)^{d_{i}}$ be the characteristic polynomial of this stratum. Then, as above, the characteristic polynomial of $\left[\Lambda_{\mathfrak{o}_{E \gamma}}, t, t-1, s_{\gamma}(\xi-\gamma)\right]$ is $\prod_{i=1}^{l} \psi_{i}(X)^{\delta_{i}}$, where $\xi=\sum_{i=1}^{l} \iota_{W^{i}}\left(\xi_{i}\right)$.

Now let $\left[\Lambda, n, t-1, \beta^{\prime}\right]$ be a simple stratum equivalent to $[\Lambda, n, t-1, \beta]$; then, if $b^{\prime}=\beta^{\prime}-\gamma=$ $\left(\beta^{\prime}-\beta\right)+(\beta-\gamma)$, we have $b^{\prime} \equiv \xi-\gamma\left(\bmod \mathfrak{a}_{1-t}\right)$ so

$$
\left[\Lambda_{\mathfrak{o}_{E_{\gamma}}}, t, t-1, s_{\gamma}\left(b^{\prime}\right)\right] \sim\left[\Lambda_{\mathfrak{o}_{E_{\gamma}}}, t, t-1, s_{\gamma}(\xi-\gamma)\right] .
$$

The former stratum is equivalent to a null or simple stratum, by [6] (2.4.1), so we deduce that $\varphi_{i}(X)=\varphi(X)$, for $1 \leq i \leq l$. 
First suppose $\varphi(X)=X$, i.e. the strata above are equivalent to null strata. Then, by [6] (2.2.1), for $1 \leq i \leq l$ there exists a conjugate $\gamma_{i}^{\prime}$ of $\gamma$ by the group $U^{1}\left(\mathfrak{A}\left(E_{i}\right)\right)$ such that $\left[\mathfrak{A}\left(E_{i}\right), n_{i},\left\lfloor\frac{t-1}{e_{i}}\right\rfloor, \gamma_{i}^{\prime}\right]$ is equivalent to $\left[\mathfrak{A}\left(E_{i}\right), n_{i},\left\lfloor\frac{t-1}{e_{i}}\right\rfloor, \beta_{i}\right]$ and we put $\gamma^{(t-1)}=\sum_{i=1}^{l} \iota_{W^{i}}\left(\gamma_{i}^{\prime}\right)$.

So we may assume $\varphi(X) \neq X$; in particular, $\left\lfloor\frac{t-1}{e_{i}}\right\rfloor=t_{i}-1$. Choose $\Phi(X) \in \mathfrak{o}_{E_{\gamma}}[X]$ such that $\varphi(X)=\Phi(X)\left(\bmod \mathfrak{p}_{E_{\gamma}}\right)$ and choose $c_{i} \in B_{\gamma}\left(E_{i}\right)=\operatorname{End}_{E_{\gamma}} E_{i}$ such that

$$
\left[\mathfrak{A}\left(E_{i}\right)_{\mathfrak{o}_{E_{\gamma}}}, t_{i}, t_{i}-1, s_{\gamma}\left(\xi_{i}-\gamma\right)\right] \sim\left[\mathfrak{A}\left(E_{i}\right)_{\mathfrak{o}_{E \gamma}}, t_{i}, t_{i}-1, c_{i}\right]
$$

and $c_{i}$ has minimal polynomial $\Phi\left(\left(\varpi_{E_{\gamma}}^{-t / g} X\right)^{e / g}\right)$, where $g=(e, t)$ and $\varpi_{E_{\gamma}}$ is a (fixed) uniformizer in $E_{\gamma}$.

We put $E_{i}^{\prime}=E_{\gamma}\left[c_{i}\right]$; these are all isomorphic to $E^{\prime}:=E_{\gamma}[c]$, where $c$ has minimal polynomial $\Phi\left(\left(\varpi_{E_{\gamma}}^{-t / g} X\right)^{e / g}\right)$. Consider the simple stratum $\left[\mathfrak{A}\left(E^{\prime}\right), n^{\prime}, t^{\prime}, \gamma\right]$ in $A\left(E^{\prime}\right)$ and the derived stratum $\left[\mathfrak{A}\left(E^{\prime}\right)_{\mathfrak{o}_{E \gamma}}, t^{\prime}, t^{\prime}-1, c\right]$, where $e^{\prime}=e\left(\Lambda^{i} \mid \mathfrak{o}_{E_{i}^{\prime}}\right)$ and $n^{\prime}=n / e^{\prime}, t^{\prime}=t / e^{\prime}$. Choose $b \in A\left(E^{\prime}\right)$ such that $\nu_{\mathfrak{A}\left(E^{\prime}\right)}(b)=-t^{\prime}$ and $s_{\gamma}^{\prime}(b)=c$, where $s_{\gamma}^{\prime}$ is a tame corestriction on $A\left(E^{\prime}\right)$ relative to $E_{\gamma} / F$; then the stratum $\left[\mathfrak{A}\left(E^{\prime}\right), n^{\prime}, t^{\prime}-1, \gamma+b\right]$ is simple, by [6] $(2.2 .3)$.

Now let $Y_{i}$ be in general position relative to $\mathfrak{A}\left(E_{i}\right)$ over $E^{\prime} \simeq E_{i}^{\prime}$ and consider the stratum $\left[\mathfrak{A}\left(E_{i}\right), n_{i}, t_{i}-1, \iota_{Y_{i}}(\gamma+b)\right]$. This is simple and, as in [6] (2.2.8), some conjugate, by the group $U^{1}\left(\mathfrak{A}\left(E_{i}\right)\right),\left[\mathfrak{A}\left(E_{i}\right), n_{i}, t_{i}-1, \gamma_{i}^{\prime}\right]$ is equivalent to $\left[\mathfrak{A}\left(E_{i}\right), n_{i}, t_{i}-1, \xi_{i}\right] \sim\left[\mathfrak{A}\left(E_{i}\right), n_{i}, t_{i}-1, \beta_{i}\right]$. Then $\gamma^{(t-1)}=\sum_{i=1}^{l} \iota_{W^{i}}\left(\gamma_{i}^{\prime}\right)$ is as required.

The previous proposition shows, in particular, that we may choose $\gamma \in \mathcal{M}$, and this is the only property of $\gamma$ which we will use in $\S \$ 3.23 .3$. Then each decomposition $V^{I_{j}}=\bigoplus_{i \in I_{j}} V^{i}$ is a decomposition of $F\left[\gamma_{j}\right]$-spaces, $1 \leq j \leq m$. If $s_{j}$ is a tame corestriction on $A^{I_{j}, I_{j}}$ relative to $F\left[\gamma_{j}\right] / F$, then $\left[\Lambda_{\mathfrak{o}_{E_{j}}}^{I_{j}}, r, r-1, s_{j}\left(\beta_{I_{j}}-\gamma_{I_{j}}\right)\right]$ is equivalent to a semisimple stratum with splitting $V^{I_{j}}=\bigoplus_{i \in I_{j}} V^{i}((\mathrm{i})$ comes from [6] (2.4.1) while (ii) follows by [6] (2.2.8)).

We also have a converse to these observations, which follows from [6] (2.2.8), (2.3.12):

Lemma 3.5 (cf. [6] (2.2.8)). Let $[\Lambda, n, r, \gamma]$ be a semisimple stratum with splitting $V=\bigoplus_{j=1}^{m} V^{j}$. Put $E_{j}=F\left[\gamma_{j}\right]$ and let $s_{j}$ be a tame corestriction on $A^{j j}$ relative to $E_{j} / F$. For $1 \leq j \leq m$, let $b_{j} \in \mathfrak{a}_{-r}^{j j}$ be such that $\left[\Lambda_{\mathfrak{o}_{E_{j}}}^{j}, r, r-, s_{j}\left(b_{j}\right)\right]$ is equivalent to a semisimple stratum and put $b=\sum_{j=1}^{m} b_{j}$. Then $[\Lambda, n, r-, \gamma+b]$ is equivalent to a semisimple stratum.

Now let $[\Lambda, n, 0, \beta]$ be a non-null semisimple stratum and put

$$
k_{0}(\beta, \Lambda)=-\min \{r \in \mathbb{Z}:[\Lambda, n, r, \beta] \text { is not semisimple }\} .
$$

Note that this is consistent with the definition for simple strata in $\$ 1.2$. There are two possibilities here:

(i) For some $i, 1 \leq i \leq l$, we have $k_{0}(\beta, \Lambda)=k_{0}\left(\beta_{i}, \Lambda^{i}\right)$. Then, putting $e_{i}=e\left(E_{i} \mid F\right)$, we have

$$
\frac{k_{0}(\beta, \Lambda)}{e\left(\Lambda \mid \mathfrak{o}_{F}\right)}=\frac{k_{F}\left(\beta_{i}\right)}{e_{i}}
$$

(ii) There exist $i, j, 1 \leq i, j \leq l$, such that $\left[\Lambda^{i}, q, r, \beta_{i}\right]$ and $\left[\Lambda^{j}, q, r, \beta_{j}\right]$ are simple and $\left[\Lambda^{i} \oplus\right.$ $\left.\Lambda^{j}, q, r, \beta_{i}+\beta_{j}\right]$ is equivalent to a simple stratum $\left[\Lambda^{i} \oplus \Lambda^{j}, q, r, \gamma\right]$, where $r=-k_{0}(\beta, \Lambda)$ and $q=$ 
$q_{i}=q_{j}$ are as in Definition 3.2. Then, by [6] (2.4.1), there exist $c_{i}, c_{j} \in E_{\gamma}=F[\gamma]$ such that $\left[\Lambda_{\mathfrak{o}_{E \gamma}}^{i}, r, r-1, s_{\gamma}\left(\gamma-\beta_{i}\right)\right] \sim\left[\overline{\Lambda_{\mathfrak{o}_{E \gamma}}^{i}}, r, r-1, c_{i}\right]$, and likewise for $j$. Since, by definition of $k_{0}(\beta, \Lambda)$, $\left[\Lambda^{i} \oplus \Lambda^{j}, q, r-1, \beta_{i}+\beta_{j}\right]$ is not equivalent to a simple stratum, the derived strata above do not have the same characteristic polynomial, by [6] (2.2.8). In particular, at least one (say for $i$ ) is fundamental and we have $r=-\nu_{\Lambda_{\mathfrak{o}_{E_{\gamma}}^{i}}}\left(c_{i}\right)$. Then, putting $e_{i}=e\left(E_{i} \mid F\right)=e\left(E_{\gamma} \mid F\right)$, we have

$$
\frac{k_{0}(\beta, \Lambda)}{e\left(\Lambda \mid \mathfrak{o}_{F}\right)}=-\frac{\nu_{E_{\gamma}}\left(c_{i}\right)}{e_{i}}
$$

Now let $\left[\Lambda^{\prime}, n^{\prime}, 0, \beta\right]$ be another semisimple stratum (with splitting $V=\bigoplus_{i=1}^{l} V^{i}$ ). Then, whichever of the two cases above occurs, we have

$$
\frac{k_{0}(\beta, \Lambda)}{e\left(\Lambda \mid \mathfrak{o}_{F}\right)}=\frac{k_{0}\left(\beta, \Lambda^{\prime}\right)}{e\left(\Lambda^{\prime} \mid \mathfrak{o}_{F}\right)}
$$

(For case (ii), note that there is an $E_{i}$-basis of $V^{i}$ which is a splitting of both $\Lambda^{i}$ and $\Lambda^{i}$; taking $W^{i}$ to be the $F$-linear span of this basis - so that it is in general position relative to both $\Lambda^{i}$ and $\Lambda^{\prime i}$ - and choosing $W^{j}$ similarly, we may use Proposition 3.4 to choose the same $\gamma$ for $\Lambda$ and $\Lambda^{\prime}$.)

\subsection{Definitions}

We continue in the situation above, so $[\Lambda, n, r-, \beta]$ is a semisimple stratum with splitting $V=$ $\bigoplus_{i=1}^{l} V^{i}$ and $[\Lambda, n, r, \beta]$ is equivalent to the semisimple stratum $[\Lambda, n, r, \gamma]$, with $\gamma \in \mathcal{M}$; we write $b=\beta-\gamma$. Let $B_{\beta}$ denote the $A$-centralizer of $\beta$; we have $B_{\beta}=\bigoplus_{i=1}^{l} B_{\beta_{i}}^{i i}$. We consider the adjoint map $a_{\beta}: x \mapsto \beta x-x \beta$, for $x \in A$. Note that the restriction of $a_{\beta}$ to $A^{i j}$ is certainly bijective for $i \neq j$. For $k \in \mathbb{Z}$ we put

$$
\mathfrak{n}_{k}(\beta, \Lambda)=\left\{a \in \mathfrak{a}_{0}: a_{\beta}(x) \in \mathfrak{a}_{k}\right\} .
$$

We clearly have $\mathfrak{n}_{k}(\beta, \Lambda)^{i i}=\mathfrak{n}_{k}\left(\beta_{i}, \Lambda^{i}\right)$, for $1 \leq i \leq l$.

Lemma 3.7. For $k \leq r$ we have

(i) for $i \neq j, \mathfrak{n}_{-k}(\beta, \Lambda)^{i j} \subset \mathfrak{a}_{r-k}$;

(ii) $\mathfrak{n}_{-k}(\beta, \Lambda)=\mathfrak{b}_{\beta, 0}+\mathfrak{n}_{-k}(\beta, \Lambda) \cap \mathfrak{a}_{r-k}$.

Proof We note first that we have $\mathfrak{n}_{-k}(\beta, \Lambda) \cap \mathfrak{a}_{r-k}=\mathfrak{n}_{-k}(\gamma, \Lambda) \cap \mathfrak{a}_{r-k}$, since, for $x \in \mathfrak{a}_{-r-k}$, $a_{\beta}(x) \equiv a_{\gamma}(x)\left(\bmod \mathfrak{a}_{-k}\right)$. Also, (ii) holds in the simple case by [6] (1.4.9) (see also [12] §4) and hence follows immediately from (i) in the general case.

Let us fix $i \neq j$. We put $q=\max \left\{q_{i}, q_{j}\right\}$ and let $t \in \mathbb{Z}$ with $r \leq t \leq q$ be minimal such that $\left[\Lambda^{i} \oplus \Lambda^{j}, q, t, \beta_{i}+\beta_{j}\right]$ is equivalent to a null or simple stratum, say $\left[\Lambda^{i} \oplus \Lambda^{j}, q, t, \zeta\right]$. Put $E=F[\zeta]$ and let $s_{\zeta}$ be a tame corestriction on $\operatorname{End}_{F}\left(V^{i} \oplus V^{j}\right)$ relative to $E / F$. Put $b=\beta_{i}+\beta_{j}-\zeta$; then, by the minimality of $t$ and [6] (2.2.8), the derived stratum $\left[\Lambda_{\mathfrak{o}_{E}}^{i} \oplus \Lambda_{\mathfrak{o}_{E}}^{j}, t, t-1, s_{\zeta}(b)\right]$ is split. We put $s=-k_{0}\left(\zeta, \Lambda^{i} \oplus \Lambda^{j}\right)$.

Now let $y \in \mathfrak{a}_{-k}^{i j}$; then $s_{\zeta}(y) \in \mathfrak{b}_{\zeta,-k}^{i j}$ so, by [7] (3.7) Lemma 4, there exists $z \in \mathfrak{b}_{\zeta, t-k}$ such that $a_{s_{\zeta}(b)}(z)=s_{\zeta}(y)$. Then $y-a_{b}(z) \in \operatorname{ker} s_{\zeta} \cap \mathfrak{a}_{-k}$ so, by [6] (1.4.10), there exists $x \in \mathfrak{n}_{-k}\left(\zeta, \Lambda^{i} \oplus \Lambda^{j}\right) \cap$ 
$\mathfrak{a}_{s-k}$ such that $a_{\zeta}(x)=y-a_{b}(z)$. Then $a_{\beta}(x+z) \equiv y\left(\bmod \mathfrak{a}_{1-k}\right)$ and, since $x+z \in \mathfrak{a}_{r-k}$, it follows easily that the image $a_{\beta}\left(\mathfrak{a}_{s-k}^{i j}\right)$ contains $\mathfrak{a}_{-k}^{i j}$. Since $a_{\beta}$ is bijective on $A^{i j}$, we get an isomorphism

$$
\mathfrak{n}_{-k}(\beta, \Lambda) \cap \mathfrak{a}_{s-k}^{i j} \stackrel{\sim}{\rightarrow} \mathfrak{a}_{-k}^{i j}
$$

Since $s>r$, we have $\mathfrak{n}_{-k}(\beta, \Lambda)^{i j} \subset \mathfrak{a}_{s-k} \subset \mathfrak{a}_{r-k}$, as required.

Now we define the orders $\mathfrak{H}(\beta, \Lambda) \subset \mathfrak{J}(\beta, \Lambda)$ inductively by

$$
\begin{aligned}
\mathfrak{H}(\beta, \Lambda) & =\mathfrak{b}_{\beta, 0}+\mathfrak{H}^{\frac{r}{2}+}(\gamma, \Lambda), \\
\mathfrak{J}(\beta, \Lambda) & =\mathfrak{b}_{\beta, 0}+\mathfrak{J}^{\frac{r}{2}}(\gamma, \Lambda),
\end{aligned}
$$

with $\mathfrak{H}(0, \Lambda)=\mathfrak{J}(0, \Lambda)=\mathfrak{a}_{0}(\Lambda)$. Note that this is consistent with the definitions of [6] $\S 3.1$ in the simple case, by op. cit. $(3.1 .9)(v),(3.1 .10)(v)$. Moreover, as in the Remark following loc. cit., to check that this definition of $\mathfrak{H}(\beta, \Lambda)$ is independent of the choice of $\gamma \in \mathcal{M}$, we need only prove:

Lemma 3.9 (cf. [6] (3.1.9)(v)). Let $\left[\Lambda, n, r-, \beta^{\prime}\right]$ be a semisimple stratum equivalent to $[\Lambda, n, r-, \beta]$ and with the same splitting. Then

$$
\mathfrak{H}^{\frac{r}{2}}\left(\beta^{\prime}, \Lambda\right)=\mathfrak{H}^{\frac{r}{2}}(\beta, \Lambda)
$$

Proof We assume in this proof that $\mathfrak{H}\left(\beta^{\prime}, \Lambda\right)$ has been defined relative to the same semisimple stratum $[\Lambda, n, r, \gamma]$; then the only possible difference between the two orders must lie in $\mathcal{M}$, since $B_{\beta}$ and $B_{\beta^{\prime}}$ are both contained in $\mathcal{M}$. But, for each $i,\left[\Lambda^{i}, n, r-, \beta_{i}^{\prime}\right]$ and $\left[\Lambda^{i}, n, r-, \beta_{i}\right]$ are equivalent simple strata so

$$
\mathfrak{H}^{\frac{r}{2}}\left(\beta^{\prime}, \Lambda\right)^{i i}=\mathfrak{H}^{\frac{r}{2}}\left(\beta_{i}^{\prime}, \Lambda^{i}\right)=\mathfrak{H}^{\frac{r}{2}}\left(\beta_{i}, \Lambda^{i}\right)=\mathfrak{H}^{\frac{r}{2}}(\beta, \Lambda)^{i i},
$$

by the simple case [6] $(3.1 .9)(v)$.

Similarly, $\mathfrak{J}(\beta, \Lambda)$ is independent of the choice of $\gamma \in \mathcal{M}$.

Lemma 3.10 (cf. [6] (3.1.10)). For $0 \leq k \leq r$, we have

(i) $\mathfrak{n}_{-\frac{k}{2}}(\beta, \Lambda) \cap \mathfrak{a}_{r-\frac{k}{2}} \subset \mathfrak{J}^{r-\frac{k}{2}}(\beta, \Lambda)$;

(ii) $\mathfrak{J}^{\frac{k}{2}}(\beta, \Lambda)$ is an $\mathfrak{n}_{-k}(\beta, \Lambda)$-bimodule.

Proof We proceed by induction on $r$, with the simple case given by [6] (3.1.10). By Lemma 3.7(ii),

$$
\begin{aligned}
\mathfrak{n}_{-\frac{k}{2}}(\beta, \Lambda) \cap \mathfrak{a}_{r-\frac{k}{2}} & =\mathfrak{n}_{-\frac{k}{2}}(\gamma, \Lambda) \cap \mathfrak{a}_{r-\frac{k}{2}} \\
& =\mathfrak{b}_{\gamma, r-\frac{k}{2}}+\mathfrak{n}_{-\frac{k}{2}}(\gamma, \Lambda) \cap \mathfrak{a}_{r-\frac{k}{2}+}
\end{aligned}
$$

Now, since $r-\frac{k}{2} \geq \frac{r}{2}$, we have that $\mathfrak{J}^{r-\frac{k}{2}}(\beta, \Lambda)=\mathfrak{J}^{r-\frac{k}{2}}(\gamma, \Lambda)$ and also $\mathfrak{b}_{\gamma, r-\frac{k}{2}} \subset \mathfrak{J}^{r-\frac{k}{2}}(\gamma, \Lambda)$, while $\mathfrak{n}_{-\frac{k}{2}}(\gamma, \Lambda) \cap \mathfrak{a}_{r-\frac{k}{2}+} \subset \mathfrak{J}^{r-\frac{k}{2}+}(\gamma, \Lambda) \subset \mathfrak{J}^{r-\frac{k}{2}}(\gamma, \Lambda)$, by induction, so (i) follows. 
We have that $\mathfrak{J}^{\frac{k}{2}}(\beta, \Lambda)=\mathfrak{b}_{\beta, \frac{k}{2}}+\mathfrak{J}^{\frac{r}{2}}(\gamma, \Lambda)$ and also $\mathfrak{n}_{-k}(\beta, \Lambda) \subset \mathfrak{n}_{-r}(\beta, \Lambda)=\mathfrak{n}_{-r}(\gamma, \Lambda)$ so that $\mathfrak{n}_{-k}(\beta, \Lambda) \mathfrak{J}^{\frac{r}{2}}(\gamma, \Lambda) \subset \mathfrak{J}^{\frac{r}{2}}(\gamma, \Lambda)$ by induction. Also, $\mathfrak{n}_{-k}(\beta, \Lambda)=\mathfrak{b}_{\beta, 0}+\mathfrak{n}_{-k}(\beta, \Lambda) \cap \mathfrak{a}_{r-k}$ by Lemma 3.7 (ii). The result now follows from (i) since

$$
\begin{aligned}
\left(\mathfrak{n}_{-k}(\beta, \Lambda) \cap \mathfrak{a}_{r-k}\right) \mathfrak{b}_{\beta, \frac{k}{2}} & \subset \mathfrak{n}_{-\frac{k}{2}}(\beta, \Lambda) \cap \mathfrak{a}_{r-\frac{k}{2}} \\
& \subset \mathfrak{J}^{r-\frac{k}{2}}(\beta, \Lambda) \subset \mathfrak{J}^{\frac{k}{2}}(\beta, \Lambda) .
\end{aligned}
$$

Similarly, $\mathfrak{n}_{-\frac{k}{2}}(\beta, \Lambda) \cap \mathfrak{a}_{r-\frac{k}{2}} \subset \mathfrak{J}^{r-\frac{k}{2}}(\beta, \Lambda)$ and $\mathfrak{H}^{\frac{k}{2}+}(\beta, \Lambda)$ is an $\mathfrak{n}_{-k}(\beta, \Lambda)$-bimodule, for $0 \leq k \leq r$.

Lemma 3.11 (cf. [6] (3.1.13)). (i) For $k<r$, we have

$$
\left(\mathfrak{n}_{-k}(\beta, \Lambda) \cap \mathfrak{a}_{r-k}\right) \mathfrak{J}^{\frac{k}{2}}(\beta, \Lambda) \subset \mathfrak{H}^{\frac{k}{2}+}(\beta, \Lambda) .
$$

(ii) For $k>0$, we have $\mathfrak{b}_{\beta, 1} \mathfrak{J}^{k}(\beta, \Lambda) \subset \mathfrak{H}^{k+1}(\beta, \Lambda)$.

(iii) For $k, l>1, \mathfrak{J}^{k}(\beta, \Lambda) \mathfrak{J}^{l}(\beta, \Lambda) \subset \mathfrak{H}^{k+l}(\beta, \Lambda)$.

(iv) For $k>0, \mathfrak{H}^{k}(\beta, \Lambda)$ is a two-sided ideal of $\mathfrak{J}(\beta, \Lambda)$.

Proof The simple case is given by [6] (3.1.13). We have $\mathfrak{J}^{\frac{k}{2}}=\mathfrak{b}_{\beta, \frac{k}{2}}+\mathfrak{J}^{\frac{r}{2}}(\gamma, \Lambda)$. Now

$$
\begin{aligned}
\left(\mathfrak{n}_{-k}(\beta, \Lambda) \cap \mathfrak{a}_{r-k}\right) \mathfrak{b}_{\beta, \frac{k}{2}} & \subset \mathfrak{n}_{-\frac{k}{2}}(\beta, \Lambda) \cap \mathfrak{a}_{r-\frac{k}{2}} \\
& \subset \mathfrak{H}^{r-\frac{k}{2}}(\beta, \Lambda) \subset \mathfrak{H}^{\frac{k}{2}+}(\beta, \Lambda),
\end{aligned}
$$

since $k<r$. On the other hand,

$$
\left(\mathfrak{n}_{-k}(\beta, \Lambda) \cap \mathfrak{a}_{r-k}\right) \mathfrak{J}^{\frac{r}{2}}(\beta, \Lambda)=\left(\mathfrak{n}_{-k}(\gamma, \Lambda) \cap \mathfrak{a}_{r-k}\right) \mathfrak{J}^{\frac{r}{2}}(\gamma, \Lambda) \subset \mathfrak{H}^{\frac{r}{2}+}(\gamma, \Lambda)
$$

by induction.

For (ii), it suffices to show that $\mathfrak{b}_{\beta, 1} \mathfrak{J}^{\frac{r}{2}}(\beta, \Lambda) \subset \mathfrak{H}(\beta, \Lambda)$, which is immediate from (i), with $k=r-1$. The remaining assertions follow, as in [6] (3.1.13).

Now, for $m \geq-1$, we put $H^{m+1}(\beta, \Lambda)=\mathfrak{H}(\beta, \Lambda) \cap U^{m+1}(\Lambda)$ and similarly for $J^{m+1}(\beta, \Lambda)$. We will usually write $J(\beta, \Lambda)=J^{0}(\beta, \Lambda)$. Note also that $H^{m+1}(\beta, \Lambda) \cap \widetilde{G}_{i}=H^{m+1}\left(\beta_{i}, \Lambda^{i}\right)$, for $1 \leq i \leq l$,

Corollary 3.12 (cf. [6] (3.1.15)). Abbreviating $H^{m}=H^{m}(\beta, \Lambda)$ and likewise for $J^{m}$, we have:

(i) for $0<m \leq \frac{r}{2}+$ and $0<l \leq \frac{r}{2}$,

$$
H^{m}=\left(U^{m}(\Lambda) \cap B_{\beta}\right) \cdot H^{\frac{r}{2}+}, \quad J^{l}=\left(U^{l}(\Lambda) \cap B_{\beta}\right) \cdot J^{\frac{r}{2}} ;
$$

(ii) for $m \geq 0, H^{m} \subset J^{m}$ and, for $m>0, H^{m} \triangleleft J$;

(iii) for $k, l>0,\left[J^{k}, J^{l}\right] \subset H^{k+l}$.

We also remark that $H(\beta, \Lambda)$ and $J(\beta, \Lambda)$ have Iwahori decompositions with respect to $M$. 
Definition 3.13 (cf. [6] (3.2.1), (3.2.3), [13] (3.11))). For $0 \leq m<r$, the $\operatorname{set} \mathcal{C}(\Lambda, m, \beta)$ of semisimple characters of $H^{m+}(\beta, \Lambda)$ is the set of characters $\theta$ such that

(i) $\left.\theta\right|_{H^{m+(\beta, \Lambda) \cap} \widetilde{G}_{i}} \in \mathcal{C}\left(\Lambda^{i}, m, \beta_{i}\right)$, for $1 \leq i \leq l$;

(ii) if $m^{\prime}=\max \left\{m, \frac{r}{2}\right\}$, the restriction $\left.\theta\right|_{H^{m^{\prime}+(\beta, \Lambda)}}$ is of the form $\theta_{0} \psi_{b}$ for some $\theta_{0} \in \mathcal{C}\left(\Lambda, m^{\prime}, \gamma\right)$, where $b=\beta-\gamma$.

Note that this too is consistent with the definitions of [6] $\S 3.2$, by [6] (3.3.20).

Remarks 3.14. (i) If $m \geq \frac{r}{2}$ then we have $H^{m+}(\beta, \Lambda)=H^{m+}(\gamma, \Lambda)$ and condition (i) of the definition is implied by (ii). In particular we have a bijection

$$
\begin{aligned}
\mathcal{C}(\Lambda, m, \gamma) & \rightarrow \mathcal{C}(\Lambda, m, \beta) ; \\
\theta & \mapsto \theta \psi_{b} .
\end{aligned}
$$

(ii) Suppose $\left[\Lambda, n, r, \gamma^{\prime}\right]$ is another semisimple stratum which is equivalent to $[\Lambda, n, r, \beta]$, with $\gamma^{\prime} \in$ $\mathcal{M}$. Then we could define the set $\mathcal{C}(\Lambda, m, \beta)$ with respect to this stratum also. However, these definitions coincide: this follows as in [6] (3.3.20) from the fact that, if $\left[\Lambda, n, r-, \beta^{\prime}\right]$ is a semisimple stratum equivalent to $[\Lambda, n, r-, \beta]$ and with the same splitting, then

$$
\mathcal{C}\left(\Lambda, \frac{r}{2}-, \beta^{\prime}\right)=\mathcal{C}\left(\Lambda, \frac{r}{2}-, \beta\right) \cdot \psi_{\beta^{\prime}-\beta}
$$

(cf. [6] (3.3.20) $(i i))$. To prove this, we assume that $\mathcal{C}\left(\Lambda, \frac{r}{2}-, \beta^{\prime}\right)$ has been defined relative to the same semisimple stratum $[\Lambda, n, r, \gamma]$; then, given $\theta \in \mathcal{C}\left(\Lambda, \frac{r}{2}-, \beta\right)$, the character $\theta \psi_{\beta^{\prime}-\beta}$ of $H^{\frac{r}{2}}(\beta, \Lambda)=H^{\frac{r}{2}}\left(\beta^{\prime}, \Lambda\right)$ certainly satisfies condition (ii) of Definition 3.13 , while condition (i) comes from the simple case [6] (3.3.20) $(i)$.

(iii) The set $\mathcal{C}(\Lambda, m, \beta)$ is indeed independent of $r$ since, if $r<-k_{0}(\beta, \Lambda)$, we may take $\gamma=\beta$ in the definitions.

Lemma 3.15. (i) Let $0 \leq m<r$ and $\theta_{0} \in \mathcal{C}\left(\Lambda, \frac{r}{2}, \gamma\right)$. For $1 \leq i \leq l$, let $\theta_{i} \in \mathcal{C}\left(\Lambda^{i}, m, \beta_{i}\right)$ be such that $\theta_{i}$ agrees with $\theta_{0} \psi_{b}$ on $H^{\frac{r}{2}+}(\beta, \Lambda) \cap \widetilde{G}_{i}$. Then there exists a unique character $\theta$ of $H^{m+}=H^{m+}(\beta, \Lambda)$ such that $\left.\theta\right|_{H^{m+} \cap M}=\bigotimes_{i=1}^{l} \theta_{i}$ and $\left.\theta\right|_{H^{\frac{r}{2}+}{ }_{(\beta, \Lambda)}}=\theta_{0} \psi_{b}$. Moreover, $\theta$ is trivial on $N_{l}$ and $N_{u}$.

(ii) Let $\theta_{0} \in \mathcal{C}\left(\Lambda, \frac{r}{2}, \gamma\right)$. Then $J(\beta, \Lambda)$ normalizes $\left.\theta_{0} \psi_{b}\right|_{H^{2}+(\gamma, \Lambda)}$.

(iii) Let $0 \leq m \leq r-1$ and $\theta \in \mathcal{C}(\Lambda, m, \beta)$. Then $\theta$ is normalized by $J(\beta, \Lambda)$.

Proof We proceed by induction, the case of a simple stratum being given by [6] (3.3.1); so we assume the results hold for $\gamma, \beta_{i}$.

(i) The characters $\theta_{0} \psi_{b}$ and $\bigotimes_{i=1}^{l} \theta_{i}$ certainly agree where they are both defined. Now $H^{m+} \cap M$ normalizes $H^{\frac{r}{2}+}(\beta, \Lambda)$ since $\left.\theta_{0} \psi_{b}\right|_{H^{\frac{r}{2}+}(\beta, \Lambda) \cap \widetilde{G}_{i}} \in \mathcal{C}\left(\Lambda^{i}, \frac{r}{2}, \beta_{i}\right)$, for $1 \leq i \leq l$. But $\theta_{0} \psi_{b}$ is trivial on $N_{l}$ and $N_{u}$ and hence $H^{m+} \cap M$ normalizes the pair $\left(H^{\frac{r}{2}+1}(\gamma, \Lambda), \theta_{0} \psi_{b}\right)$, by Corollary 2.2. The assertions are now clear.

(ii) For $j \in J^{\frac{r}{2}}(\gamma, \Lambda)$, this is implied by (iii) for $\gamma$ and, as in (i), $J \cap M$ normalizes the pair $\left(H^{\frac{r}{2}+}(\gamma, \Lambda), \theta_{0} \psi_{b}\right)$.

(iii) For $m \geq \frac{r}{2}$, this follows from (ii) so assume $m<\frac{r}{2}$; then, for $j \in J(\beta, \Lambda), h \in H^{\frac{r}{2}+}(\beta, \Lambda$ ) we have $\theta\left(j h j^{-1}\right)=\theta(h)$. Also, for $j \in J(\beta, \Lambda) \cap N_{l}, h \in H^{m+}(\beta, \Lambda) \cap M$, we have $\theta([j, h])=1$ since $[j, h] \in H^{m+}(\beta, \Lambda) \cap N_{l} \subset \operatorname{ker} \theta$, and likewise for $N_{u}$. Hence we need only check that $J(\beta, \Lambda) \cap M$ normalizes $\bigotimes_{i=1}^{l} \theta_{i}$, which follows from the simple case. 
For $m \leq r$, we put

$$
\mathfrak{m}_{m}(\beta, \Lambda)=\mathfrak{n}_{-m}(\beta, \Lambda) \cap \mathfrak{a}_{r-m}+\mathfrak{J}^{\frac{r}{2}}(\beta, \Lambda) .
$$

Note that for $m \leq \frac{r}{2}$ we have $\mathfrak{m}_{m}(\beta, \Lambda)=\mathfrak{J}^{\frac{r}{2}}(\beta, \Lambda)$. On the other hand, for $m>\frac{r}{2}, \mathfrak{n}_{-m}(\beta, \Lambda) \cap$ $\mathfrak{a}_{r-m}=\mathfrak{n}_{-m}(\gamma, \Lambda) \cap \mathfrak{a}_{r-m}=\mathfrak{b}_{\gamma, r-m}+\mathfrak{n}_{-m}(\beta, \Lambda) \cap \mathfrak{a}_{r-m+}$, by Lemma 3.7(ii); hence $\mathfrak{m}_{m}(\beta, \Lambda)=$ $\mathfrak{b}_{\gamma, r-m}+\mathfrak{m}_{m}(\gamma, \Lambda)$.

We note that, if $[\Lambda, n, r-, \beta]$ is simple, then $\mathfrak{m}_{m}(\beta, \Lambda)$ is not the same as the lattice $\mathfrak{M}_{m}(\beta, \Lambda)$ defined in [7] (5.6), unless $\lceil r\rceil=-k_{0}(\beta, \Lambda)$; otherwise, we have $\mathfrak{m}_{m}(\beta, \Lambda)=\mathfrak{b}_{\beta, r-m}+\mathfrak{M}_{m}(\beta, \Lambda)$ so $\mathfrak{m}_{m}(\beta, \Lambda)$ does in fact depend on $r$, though only in a rather trivial way.

For $0 \leq m<r$, we put $\Gamma_{m}(\beta, \Lambda)=1+\mathfrak{m}_{m}(\beta, \Lambda)$ and we also put $\Gamma_{r}(\beta, \Lambda)=\mathfrak{m}_{r}(\beta, \Lambda)^{\times}$. Hence we have, for $m>\frac{r}{2}, \Gamma_{m}(\beta, \Lambda)=\left(U_{r-m}(\Lambda) \cap B_{\gamma}\right) \Gamma_{m}(\gamma, \Lambda)$, with the first factor normalizing the second.

Lemma 3.16. Let $0 \leq m<r$ and $\theta \in \mathcal{C}(\Lambda, m, \beta)$. Then $\theta$ is normalized by $\Gamma_{m}(\beta, \Lambda)$.

Proof For $m \leq \frac{r}{2}$ this is weaker than Lemma 3.15 so suppose $m>\frac{r}{2}$ so that $\Gamma_{m}(\beta, \Lambda)=$ $\left(U_{r-m}(\Lambda) \cap B_{\gamma}\right) \Gamma_{m}(\gamma, \Lambda)$ and $\theta=\theta_{0} \psi_{b}$. Then $\Gamma_{m}(\beta, \Lambda)$ normalizes $\theta_{0}$, by induction and Lemma 3.15. But we have $\Gamma_{m}(\beta, \Lambda) \subset U_{r-m}(\Lambda)$ so $\Gamma_{m}(\beta, \Lambda)$ clearly normalizes $\psi_{b}$ also.

\subsection{Intertwining}

In this section we calculate the intertwining of semisimple characters. We remark that we certainly have $B_{\beta}^{\times} \subset I_{M}\left(\left.\theta\right|_{H^{m+}(\beta, \Lambda) \cap M}\right)$, by the simple case [6] (3.3.2), and hence $B_{\beta}^{\times} \subset I_{\widetilde{G}}(\theta)$ by Lemma 2.1. Then, by Lemma 3.16, we certainly have

$$
I_{\widetilde{G}}(\theta) \supset \Gamma_{m}(\beta, \Lambda) B_{\beta}^{\times} \Gamma_{m}(\beta, \Lambda) .
$$

We will show that we in fact have equality here. First we need some exact sequences.

Lemma 3.17 (cf. [7] (6.3) Lemma). Let $0 \leq m<r$. The sequence

$$
0 \rightarrow \mathfrak{b}_{\beta, r-m} \rightarrow \mathfrak{m}_{m}(\beta, \Lambda) \stackrel{a_{\beta}}{\rightarrow}\left(\mathfrak{H}^{m+}(\beta, \Lambda)\right)^{*} \cap \operatorname{im} a_{\beta} \rightarrow 0
$$

is exact. Moreover, if $h \in B_{\beta}^{\times}$and $0 \rightarrow \mathfrak{l}_{1} \rightarrow \mathfrak{l}_{2} \rightarrow \mathfrak{l}_{3} \rightarrow 0$ denotes the above sequence, then the sequence

$$
0 \rightarrow h^{-1} \mathfrak{l}_{1}^{i j} h+\mathfrak{l}_{1}^{i j} \rightarrow h^{-1} \mathfrak{l}_{2}^{i j} h+\mathfrak{l}_{2}^{i j} \rightarrow h^{-1} \mathfrak{r}_{3}^{i j} h+\mathfrak{l}_{3}^{i j} \rightarrow 0
$$

is also exact, for any $1 \leq i, j \leq l$.

Proof In the $(i, i)$-blocks, the sequences are exact by the simple case, 7] (6.3) Lemma (recall that $\left.\mathfrak{m}_{m}(\beta, \Lambda)^{i i}=\mathfrak{b}_{\beta_{i}, r-m}+\mathfrak{M}_{m}\left(\beta_{i}, \Lambda^{i}\right)\right)$, while in the $(i, j)$-blocks, $i \neq j$, the exactness of the first sequence says that $a_{\beta}$ induces an isomorphism

$$
\mathfrak{m}_{m}(\beta, \Lambda)^{i j} \stackrel{\sim}{\rightarrow}\left(\mathfrak{H}^{m+}(\beta, \Lambda)\right)^{* i j} .
$$

We put $q=\max \left\{q_{i}, q_{j}\right\}$ and let $r \leq t \leq q$ be minimal such that $\left[\Lambda^{i} \oplus \Lambda^{j}, q, t, \beta_{i}+\beta_{j}\right]$ is equivalent to a null or simple stratum, say $\left[\Lambda^{i} \oplus \Lambda^{j}, q, t, \zeta\right]$. Put $E=F[\zeta]$ and let $s_{\zeta}$ be a tame corestriction on $\operatorname{End}_{F}\left(V^{i} \oplus V^{j}\right)$ relative to $E / F$. Also put $b=\beta_{i}+\beta_{j}-\zeta$ and $s=-k_{0}(\zeta, \Lambda)$. The derived stratum $\left[\Lambda_{\mathfrak{o}_{E}}^{i} \oplus \Lambda_{\mathfrak{o}_{E}}^{j}, t, t-1, s_{\zeta}(b)\right]$ is split. 
We have

$$
\begin{aligned}
& \mathfrak{m}_{m}(\beta, \Lambda)^{i j}=\mathfrak{n}_{-m}\left(\zeta, \Lambda^{i} \oplus \Lambda^{j}\right) \cap \mathfrak{a}_{s-m}^{i j}+\mathfrak{J}^{\frac{s}{2}}\left(\zeta, \Lambda^{i} \oplus \Lambda^{j}\right)^{i j}, \\
& \left(\mathfrak{H}^{m+}(\beta, \Lambda)\right)^{* i j}=\left(\mathfrak{H}^{m^{\prime}+}\left(\zeta, \Lambda^{i} \oplus \Lambda^{j}\right)\right)^{* i j},
\end{aligned}
$$

where $m^{\prime}=\max \left\{m, \frac{s}{2}\right\}$. Then

$$
a_{\zeta}\left(\mathfrak{m}_{m}(\beta, \Lambda)^{i j}\right) \subset \mathfrak{a}_{-m}^{i j}+a_{\zeta}\left(\mathfrak{J}^{\frac{s}{2}}\left(\zeta, \Lambda^{i} \oplus \Lambda^{j}\right)^{i j}\right),
$$

which is contained in $\left(\mathfrak{H}^{m+}(\beta, \Lambda)\right)^{* i j}$ by the simple case, and

$$
a_{b}\left(\mathfrak{m}_{m}(\beta, \Lambda)^{i j}\right) \subset \mathfrak{a}_{s-t-m}^{i j}+\mathfrak{a}_{\frac{s}{2}-t}^{i j} \subset \mathfrak{a}_{-m^{\prime}}^{i j} .
$$

Hence we have $a_{\beta}\left(\mathfrak{m}_{m}(\beta, \Lambda)^{i j}\right) \subset\left(\mathfrak{H}^{m+}(\beta, \Lambda)\right)^{* i j}$ so we need only check surjectivity.

Let $y \in\left(\mathfrak{H}^{m+}(\beta, \Lambda)\right)^{* i j}$; then $s_{\zeta}(y) \in \mathfrak{b}_{\zeta,-m^{\prime}}^{i j}$ so, by [7] (3.7) Lemma 4, there exists $z \in \mathfrak{b}_{\zeta, t-m^{\prime}}^{i j}$ such that $s_{\zeta}(y)=a_{s_{\zeta}(b)}(z)$. Then $y-a_{b}(z) \in\left(\mathfrak{H}^{m+}(\beta, \Lambda)\right)^{* i j} \cap$ ker $s_{\zeta}$ so, by the simple case, there exists $x \in \mathfrak{m}_{m^{\prime}}(\beta, \Lambda)^{i j}=\mathfrak{J}^{\frac{s}{2}}\left(\zeta, \Lambda^{i} \oplus \Lambda^{j}\right)^{i j}$ such that $a_{\zeta}(x)=y-a_{b}(z)$. Then $a_{\beta}(x+z)=y+a_{b}(x)$ and we have $a_{b}(x) \in \mathfrak{a}_{\frac{s}{2}-t}^{i j} \subset \mathfrak{a}_{-\frac{s}{2}}^{i j}$. Then, by 3.8 with $k=\frac{s}{2}$, there exists $v \in \mathfrak{n}_{-\frac{s}{2}}(\beta, \Lambda) \cap \mathfrak{a}_{\frac{s}{2}}^{i j} \subset$ $\mathfrak{J}^{\frac{s}{2}}\left(\zeta, \Lambda^{i} \oplus \Lambda^{j}\right)^{i j}$ such that $a_{\beta}(z)=a_{b}(x)$. Then we have $y=a_{\beta}(x+z-v)$.

The exactness of the second sequence is now clear, since all the $\mathfrak{l}_{k}$ are $\mathfrak{b}_{\beta, 0}$-modules and the canonical projections $\mathbf{1}^{i}$ lie in $\mathfrak{b}_{\beta, 0}$.

Similarly, we have an exact sequence

$$
0 \rightarrow \mathfrak{b}_{\beta, m+} \rightarrow \mathfrak{H}^{m+}(\beta, \Lambda) \stackrel{a_{\beta}}{\rightarrow}\left(\mathfrak{m}_{m}(\beta, \Lambda)\right)^{*} \cap \operatorname{im} a_{\beta} \rightarrow 0 .
$$

In particular, with $m=\frac{r}{2}$ we have

$$
0 \rightarrow \mathfrak{b}_{\beta, \frac{r}{2}+} \rightarrow \mathfrak{H}^{\frac{r}{2}+}(\beta, \Lambda) \stackrel{a_{\beta}}{\rightarrow}\left(\mathfrak{J}^{\frac{r}{2}}(\beta, \Lambda)\right)^{*} \cap \operatorname{im} a_{\beta} \rightarrow 0 .
$$

Lemma 3.19. Let $0 \leq m<r, g \in \Gamma_{m}(\beta, \Lambda), h \in H^{\frac{m}{2}+}$ and $\theta \in \mathcal{C}\left(\Lambda, \frac{r}{2}, \beta\right)$. Then the commutator $[g, h] \in H^{\frac{r}{2}+}(\beta, \Lambda)$ and

$$
\theta[g, h]=\psi_{g^{-1} \beta g-\beta}(h) .
$$

Proof We proceed by induction, the simple case being given by [6] (3.2.11). We suppose first that $m>\frac{r}{2}$ so that we can write $g=u g^{\prime}$ with $u \in\left(U_{r-m}(\Lambda) \cap B_{\gamma}\right), g^{\prime} \in \Gamma_{m}(\gamma, \Lambda)$. Also, if $[\Lambda, n, r, \gamma]$ is a semisimple stratum equivalent to $[\Lambda, n, r, b]$, then $\theta=\theta_{0} \psi_{b}$, for some $\theta_{0} \in \mathcal{C}\left(\Lambda, \frac{r}{2}, \gamma\right)$ and $b=\beta-\gamma$. Then

$$
\begin{aligned}
\theta_{0}[g, h]=\theta_{0}\left[u g^{\prime}, h\right] & =\theta_{0}\left[g^{\prime}, h\right] & & \text { since } u \text { normalizes } \theta_{0} \\
& =\psi_{g^{\prime-1} \gamma g^{\prime}-\gamma}(h) & & \text { by induction } \\
& =\psi_{g^{-1} \gamma g-\gamma}(h) & & \text { since } u \text { commutes with } \gamma .
\end{aligned}
$$

We easily see that $\psi_{b}[g, h]=\psi_{g^{-1} b g-b}(h)$ so the result holds for $m>\frac{r}{2}$.

If $m \leq \frac{r}{2}$ then $g \in \Gamma_{m}=J^{\frac{r}{2}}$ normalizes $\theta$. Then the result follows from the fact that $a_{\beta}\left(\mathfrak{m}_{m}(\beta, \Lambda)\right) \subset$ $\left(\mathfrak{H}^{\frac{r}{2}+}(\beta, \Lambda)\right)^{*}$, from Lemma 3.17 . 
Corollary 3.20. Let $0 \leq m<r, g \in \Gamma_{m}(\beta, \Lambda)$ and $\theta \in \mathcal{C}\left(\Lambda, \frac{m}{2}, \beta\right)$. Then $g$ normalizes $H^{\frac{m}{2}+}(\beta, \Lambda)$ and

$$
\theta^{g}=\theta \psi_{g^{-1} \beta g-\beta}
$$

Proof $\mathfrak{H}^{\frac{m}{2}+}$ is an $\mathfrak{n}_{-m^{-}}$-bimodule so $g$ certainly normalizes $H^{\frac{m}{2}+}$ and, for $h \in H^{\frac{m}{2}+}, \theta^{g}(h)=$ $\theta(h) \theta([g, h])$. But $[g, h] \in H^{\frac{r}{2}+}$ so $\theta([g, h])$ depends only on the restriction $\theta \mid H^{\frac{r}{2}+}$ and, by Lemma 3.19, $\theta([g, h])=\psi_{g^{-1} \beta g-\beta}(h)$, as required.

Corollary 3.21. Let $0 \leq m<r, g \in \Gamma_{m+}(\beta, \Lambda) B_{\beta}^{\times} \Gamma_{m+}(\beta, \Lambda)$ and $\theta \in \mathcal{C}(\Lambda, m, \beta)$. Then, as characters of $H^{m+}(\beta, \Lambda) \cap g^{-1} H^{m+}(\beta, \Lambda) g$, we have

$$
\theta^{g}=\theta \psi_{g^{-1}} \beta g-\beta .
$$

Proof For $g \in \Gamma_{m+}$, this is given by Corollary 3.20. Now consider $g=y b h$, with $y, h \in \Gamma_{m+}$, $b \in B_{\beta}^{\times}$. Then, since $\Gamma_{m+}$ normalizes $H^{m+}$, for any $x \in H^{m+} \cap g^{-1} H^{m+} g$, we also have $x \in$ $h^{-1} b^{-1} H^{m+} b h$ and $x \in h^{-1} H^{m+} h$. Hence we have

$$
\begin{aligned}
\theta^{g}(x)=\theta^{y}\left(b h x h^{-1} b^{-1}\right) & =\theta\left(b h x h^{-1} b^{-1}\right) \psi_{y^{-1} \beta y-\beta}\left(b h x h^{-1} b^{-1}\right) \\
& =\theta\left(h x h^{-1}\right) \psi_{g^{-1} \beta g-h^{-1} \beta h}(x) \\
& =\theta(x) \psi_{h^{-1} \beta h-\beta}(x) \psi_{g^{-1} \beta g-h^{-1} \beta h}(x) \\
& =\theta(x) \psi_{g^{-1} \beta g-\beta}(x)
\end{aligned}
$$

as required.

Theorem 3.22 (cf. [6] (3.3.2), [7] (6.4)). Let $0 \leq m<r$ and let $\theta \in \mathcal{C}(\Lambda, m, \beta)$. Then we have

$$
I_{\widetilde{G}}(\theta)=\Gamma_{m}(\beta, \Lambda) B_{\beta}^{\times} \Gamma_{m}(\beta, \Lambda) .
$$

Proof We proceed by induction on $r$, the simple case being given by [6] (3.3.2). Further, the result for $m<\frac{r}{2}$ follows from the case $m=\left\lfloor\frac{r}{2}\right\rfloor$ since then $\mathfrak{m}_{m}(\beta, \Lambda)=\mathfrak{m}_{\left\lfloor\frac{r}{2}\right\rfloor}(\beta, \Lambda)$. So we may assume $m \geq\left\lfloor\frac{r}{2}\right\rfloor$. By Lemma 3.16. Lemma 2.1 and the simple case, we need only show that $I_{\widetilde{G}}(\theta) \subset \Gamma_{m} M \Gamma_{m}$.

Since the result for all $m$ follows from that for integral $m$, we suppose $m \in \mathbb{Z}$ (in particular, we will write $m+1$ for $m+$, etc.). We proceed by induction on $m$, beginning with the case " $m=r$ ". That is, we show

$$
I_{\widetilde{G}}\left(\left.\theta\right|_{H^{r+1}(\beta, \Lambda)}\right)=\Gamma_{r}(\beta, \Lambda) B_{\beta}^{\times} \Gamma_{r}(\beta, \Lambda) .
$$

But this is immediate from induction on $r$, since $\left.\theta\right|_{H^{r+1}(\beta, \Lambda)} \in \mathcal{C}(\Lambda, r, \beta)$ and $B_{\beta}^{\times} \subset\left(U_{0}(\Lambda) \cap\right.$ $\left.B_{\beta}\right) M\left(U_{0}(\Lambda) \cap B_{\beta}\right)$. So we suppose $m<r$ and we have the result for $m+1$. Let $g \in I_{\widetilde{G}}(\theta)$; then $g$ certainly intertwines the restriction of $\theta$ to $H^{m+2}(\beta, \Lambda)$ so we can write $g=y h y^{\prime}$, with $y, y^{\prime} \in \Gamma_{m+1}$ and $h \in B_{\beta}^{\times}$. Also, by Lemma 3.21, we have

$$
\theta^{g}=\theta \psi_{g^{-1} \beta g-\beta} \quad \text { as characters of } H^{m+1}(\beta, \Lambda) \cap g^{-1} H^{m+1}(\beta, \Lambda) g .
$$


Now we proceed by induction on $l$, the case $l=1$ being the simple case. Let $V=W^{1} \oplus W^{2}$ be a coarsening of the splitting $V=\bigoplus_{i=1}^{l} V^{i}$. We now use our block notation with respect this new splitting (so $A^{21}$ denotes $\operatorname{Hom}_{F}\left(W^{1}, W^{2}\right)$, etc.), but denote the Levi subgroup and unipotent radicals $M^{\prime}, N_{l}^{\prime}, N_{u}^{\prime}$. By induction, we assume that the result holds for $\left.\theta\right|_{\widetilde{G}^{i}} \in \mathcal{C}\left(\Lambda^{i}, m, \beta_{i}\right), i=1,2$. Consider the restriction of $\theta$ to the group

$$
K_{l}=1+\mathfrak{k}_{l}, \quad \mathfrak{k}_{l}=\left(\begin{array}{cc}
\mathfrak{H}^{m+2} & \mathfrak{H}^{m+1} \\
\mathfrak{H}^{m+2} & \mathfrak{H}^{m+2}
\end{array}\right)
$$

Then $g$ intertwines $\theta$ on $K_{l}$ so we have

$$
g^{-1}\left(\beta+\mathfrak{k}_{l}^{*}\right) g \cap\left(\beta+\mathfrak{k}_{l}^{*}\right) \neq \emptyset .
$$

Write $y=n_{y} m_{y} l_{y}$, with $n_{y}=1+y_{n} \in N_{u}^{\prime} \cap \Gamma_{m+1}, m_{y} \in M^{\prime} \cap \Gamma_{m+1}$ and $l_{y}=1+y_{l} \in N_{l}^{\prime} \cap \Gamma_{m+1}$; likewise, $y^{\prime}=l_{y}^{\prime} m_{y}^{\prime} n_{y}^{\prime}$. Now $a_{\beta}\left(y_{n}\right) \in \mathfrak{k}_{l}^{*}$ so $n_{y}^{-1}\left(\beta+\mathfrak{k}_{l}^{*}\right) n_{y}=\beta+\mathfrak{k}_{l}^{*}$, and likewise for $n_{y}^{\prime}$. The same is also true for $m_{y}, m_{y}^{\prime}$ since they normalize $\left.\theta\right|_{K_{l}}$ by Corollary 2.2. Hence $g^{\prime}=l_{y} h l_{y}^{\prime}$ intertwines the $\operatorname{coset} \beta+\mathfrak{k}_{l}^{*}$, that is

$$
h^{-1} a_{\beta}\left(y_{l}\right) h+a_{\beta}\left(y_{l}^{\prime}\right) \equiv 0 \quad\left(\bmod h^{-1} \mathfrak{k}_{l}^{*} h+\mathfrak{k}_{l}^{*}\right) .
$$

This is certainly satisfied in all blocks except possibly the (2,1)-block, where we have

$$
h^{-1} a_{\beta}\left(y_{l}\right) h+a_{\beta}\left(y_{l}^{\prime}\right) \equiv 0 \quad\left(\bmod \left(h^{-1} \mathfrak{k}_{l}^{*} h+\mathfrak{k}_{l}^{*}\right) \cap A^{21}\right) .
$$

By Lemma 3.17, there exist $z_{l}, z_{l}^{\prime} \in \mathfrak{m}_{m}^{21}$ such that

$$
a_{\beta}\left(h^{-1} y_{l} h+y_{l}^{\prime}\right)=a_{\beta}\left(h^{-1} z_{l} h+z_{l}^{\prime}\right) .
$$

Then, by the injectivity of $a_{\beta}$ on $A^{21}$, we have $g^{\prime}=\left(1+z_{l}\right) h\left(1+z_{l}^{\prime}\right)$.

Now the fact that $\Gamma_{m+1}$ normalizes $\Gamma_{m}$ implies that, absorbing factors into $\Gamma_{m}$, we may assume $g=n_{y} m_{y} h m_{y}^{\prime} n_{y}^{\prime}$. Similarly, by considering the restriction of $\theta$ to

$$
K_{u}=\left(\begin{array}{cc}
1+\mathfrak{H}^{m+2} & \mathfrak{H}^{m+2} \\
\mathfrak{H}^{m+1} & 1+\mathfrak{H}^{m+2}
\end{array}\right)
$$

(that is, reversing the roles of the $(1,2)$ - and $(2,1)$-blocks) we reduce to the case $g=m_{y} h m_{y}^{\prime} \in M^{\prime}$ so the result holds by the inductive hypothesis.

\subsection{Heisenberg extension}

We continue with the notation of the previous section, so $[\Lambda, n, r-, \beta]$ is a semisimple stratum, $[\Lambda, n, r, \gamma]$ is a semisimple stratum equivalent to $[\Lambda, n, r, \beta]$ and we put $b=\beta-\gamma$.

Lemma 3.23 (cf. [6] (3.2.8)). Let $0 \leq m<r, \theta \in \mathcal{C}(\Lambda, m, \beta)$ and let $j \in J^{k}(\beta, \Lambda), j^{\prime} \in J^{l}(\beta, \Lambda)$ with $k+l>m$. Then $\left[j, j^{\prime}\right] \in H^{m+}(\beta, \Lambda)$ and

$$
\theta\left[j, j^{\prime}\right]=\psi_{j^{-1} \beta j-\beta}\left(j^{\prime}\right)
$$


Proof The first assertion is Corollary 3.12(iv). We proceed by induction on $r$ but first reduce to the case $k, l \geq \frac{r}{2}$. Put $k^{\prime}=\max \left\{k, \frac{r}{2}\right\}, l^{\prime}=\max \left\{l, \frac{r}{2}\right\}$; then we may write $j=u h$, with $u \in U_{k}(\Lambda) \cap B_{\beta}^{\times}$and $h \in J^{k^{\prime}}(\beta, \Lambda)$, and likewise $j^{\prime}=u^{\prime} h^{\prime}$. Then

$$
\theta\left[j, j^{\prime}\right]=\theta\left[u, h j^{\prime} h^{-1}\right] \theta\left[h, h^{\prime}\right] \theta\left[h^{\prime} h h^{\prime-1}, u^{\prime}\right] .
$$

Let $\tilde{\theta} \in \mathcal{C}\left(\Lambda_{\tilde{\theta}}, k-, \beta\right)$ extend $\theta$; then $u \in H^{k}(\beta, \Lambda)$ and $\theta\left[u, h j^{\prime} h^{-1}\right]=\widetilde{\theta}\left[u, h j^{\prime} h^{-1}\right]=1$, since $J(\beta, \Lambda)$ normalizes $\widetilde{\theta}$. Similarly, $\theta\left[h^{\prime} h h^{\prime-1}, u^{\prime}\right]=1$ so we have

$$
\theta\left[j, j^{\prime}\right]=\theta\left[h, h^{\prime}\right]
$$

On the other hand,

$$
\psi_{j^{-1} \beta j-\beta}\left(j^{\prime}\right)=\psi_{h^{-1} \beta h-\beta}\left(u^{\prime} h^{\prime}\right),
$$

since $u$ commutes with $\beta$. We write $h=1+x, u^{\prime}=1+y$, with $x \in \mathfrak{J}^{k^{\prime}}(\beta, \Lambda), y \in \mathfrak{b}_{\beta, l}$. Then

$$
\psi_{h^{-1} \beta h-\beta}\left(u^{\prime}\right)=\psi_{F} \circ \operatorname{tr}\left(a_{\beta}(x) y-x(1+x)^{-1} a_{\beta}(x) y\right),
$$

where $\operatorname{tr}$ is $\operatorname{tr}_{A / F}$. Now $\psi_{F} \circ \operatorname{tr}\left(a_{\beta}(x) y\right)=\psi_{F} \circ \operatorname{tr}\left(-x a_{\beta}(y)\right)=1$ as $y$ commutes with $\beta$. Also

$$
\psi_{F} \circ \operatorname{tr}\left(x(1+x)^{-1} a_{\beta}(x) y\right)=\psi_{F} \circ \operatorname{tr}\left(a_{\beta}(x) y x(1+x)^{-1}\right)
$$

and $y x(1+x)^{-1} \in \mathfrak{b}_{\beta, l} \mathfrak{J}^{k^{\prime}}(\beta, \Lambda) \subset \mathfrak{H}^{\frac{r}{2}+}(\beta, \Lambda)$. But $a_{\beta}(x) \in\left(\mathfrak{H}^{\frac{r}{2}+}(\beta, \Lambda)\right)^{*}$, by Lemma 3.17, so altogether we have

$$
\psi_{j^{-1} \beta j-\beta}\left(j^{\prime}\right)=\psi_{h^{-1} \beta h-\beta}\left(h^{\prime}\right)
$$

and we have reduced to the case $k, l \geq \frac{r}{2}$. Indeed, we may (and do) assume $k=l=\frac{r}{2}$ so $\left[j, j^{\prime}\right] \in H^{r}(\beta, \Lambda)$.

We have

$$
\theta\left[j, j^{\prime}\right]=\theta_{0}\left[j, j^{\prime}\right] \psi_{b}\left[j, j^{\prime}\right]
$$

for some $\theta_{0} \in \mathcal{C}(\Lambda, r-, \gamma)$, and $\theta_{0}\left[j, j^{\prime}\right]=\psi_{j^{-1} \gamma j-\gamma}\left(j^{\prime}\right)$ by induction. But it is straightforward that $\psi_{b}\left[j, j^{\prime}\right]=\psi_{j^{-1} b j-b}\left(j^{\prime}\right)$ so the result follows.

Proposition 3.24 (cf. [6] (3.4.1)). Let $0<m \leq r$ and let $\theta \in \mathcal{C}(\Lambda, m-, \beta)$. The pairing

$$
\boldsymbol{k}_{\theta}:\left(g, g^{\prime}\right) \mapsto \theta\left[g, g^{\prime}\right], \quad g, g^{\prime} \in J^{m}(\beta, \Lambda)
$$

induces a nondegenerate alternating bilinear form

$$
J^{m}(\beta, \Lambda) / H^{m}(\beta, \Lambda) \times J^{m}(\beta, \Lambda) / H^{m}(\beta, \Lambda) \rightarrow \mathbb{C}^{\times} .
$$

Proof As in [6] (3.4.1), we need only show that

$$
\theta\left[g, g^{\prime}\right]=1 \quad \forall g^{\prime} \in J^{m}(\beta, \Lambda) \quad \Longleftrightarrow \quad g \in H^{m}(\beta, \Lambda),
$$

the implication $\Leftarrow$ being immediate, from Lemma 3.15 (iii).

We proceed as usual by induction, the simple case being [6] (3.4.1). Suppose first $m>\frac{r}{2}$; then $\theta=\theta_{0} \psi_{b}$, for some $\theta_{0} \in \mathcal{C}(\Lambda, m-, \gamma)$, and, for $g, g^{\prime} \in J^{m}(\beta, \Lambda)$, we have $\left[g, g^{\prime}\right] \in U^{r+}(\Lambda) \subset$ ker $\psi_{b}$. 
Hence $\boldsymbol{k}_{\theta}=\boldsymbol{k}_{\theta_{0}}$ and the result follows from induction, since $J^{m}(\beta, \Lambda)=J^{m}(\gamma, \Lambda)$, and likewise for $H^{m}$.

Now suppose $m \leq \frac{r}{2}$ and write $g=1+x, g^{\prime}=1+y$, with $x, y \in \mathfrak{J}^{m}(\beta, \Lambda)$. By Lemma 3.23, we have

$$
\theta\left[g, g^{\prime}\right]=\psi_{g^{-1} \beta g-\beta}\left(g^{\prime}\right)=\psi_{F} \circ \operatorname{tr}\left((1+x)^{-1} a_{\beta}(x) y\right)=1,
$$

where $\operatorname{tr}$ is $\operatorname{tr}_{A / F}$. This is true for all $y \in \mathfrak{J}^{m}(\beta, \Lambda)$ so we have $(1+x)^{-1} a_{\beta}(x) \in\left(\mathfrak{J}^{m}(\beta, \Lambda)\right)^{*} \subset$ $\left(\mathfrak{J}^{\frac{r}{2}}(\beta, \Lambda)\right)^{*}$. Then $a_{\beta}(x) \in\left(\mathfrak{J}^{\frac{r}{2}}(\beta, \Lambda)\right)^{*} \cap \operatorname{im} a_{\beta}$ so, by 3.18 , we have $x \in\left(B_{\beta}+\mathfrak{H}^{\frac{r}{2}+}(\beta, \Lambda)\right) \cap$ $\mathfrak{J}^{m}(\beta, \Lambda)=\mathfrak{H}^{m}(\beta, \Lambda)$ as required.

Corollary 3.25. Let $0 \leq m<r$ and let $\theta \in \mathcal{C}(\Lambda, m, \beta)$. Then there exists a unique irreducible representation $\eta$ of $J^{m+}(\beta, \Lambda)$ which contains $\theta$. Moreover, $\operatorname{dim} \eta=\left(J^{m+}: H^{m+}\right)^{\frac{1}{2}}$ and $I_{\widetilde{G}}(\eta)=$ $\Gamma_{m} B_{\beta}^{\times} \Gamma_{m}$.

\subsection{Transfer property}

In this section we extend the transfer property [6] (3.6.1) to semisimple strata. We continue with a semisimple stratum $[\Lambda, n, r-, \beta]$ with splitting $V=\bigoplus_{i=1}^{l} V^{i}$ and let $\theta \in \mathcal{C}(\Lambda, m, \beta), 0 \leq m<r$. We assume moreover that $r=-k_{0}(\beta, \Lambda)$ (see (3.6)) and let $[\Lambda, n, r, \gamma]$ be a semisimple stratum equivalent to $[\Lambda, n, r, \beta]$ chosen as in Proposition 3.4. Put $m_{0}=\max \left\{m, \frac{r}{2}\right\}$; then we have

$$
\begin{cases}\left.\theta\right|_{\widetilde{G}_{i}}=\theta_{i}, & \text { for some } \theta_{i} \in \mathcal{C}\left(\Lambda^{i}, m, \beta_{i}\right), \\ \left.\theta\right|_{H^{m_{0}}+(\beta, \Lambda)}=\theta_{0} \psi_{\gamma-\beta}, & \text { for some } \theta_{0} \in \mathcal{C}\left(\Lambda, m_{0}, \gamma\right) .\end{cases}
$$

Now let $\left[\Lambda^{\prime}, n^{\prime}, r^{\prime}-, \beta\right]$ be another semisimple stratum, $r^{\prime}=-k_{0}\left(\beta, \Lambda^{\prime}\right)$. Let $m^{\prime} \in \mathbb{Z}$ be such that $\left\lfloor\frac{m^{\prime}}{e\left(\Lambda^{\prime} \mid \mathfrak{o}_{F}\right)}\right\rfloor=\left\lfloor\frac{m}{e\left(\Lambda \mid \mathfrak{o}_{F}\right)}\right\rfloor$ and $\theta^{\prime} \in \mathcal{C}\left(\Lambda^{\prime}, m^{\prime}, \beta^{\prime}\right)$. For each $i$, there is an $E_{i}$-basis of $V^{i}$ which is a splitting of both $\Lambda^{i}$ and $\Lambda^{\prime i}$. Taking $W^{i}$ to be the $F$-linear span of this basis (so that it is in general position relative to both $\Lambda^{i}$ and $\Lambda^{\prime i}$ ), we may use Proposition 3.4 to choose $\gamma$ as above in $\prod \iota_{W^{i}}\left(\mathfrak{K}\left(\mathfrak{A}\left(E_{i}\right)\right)\right.$ ) (with notation as in 3.4). In particular, we may assume that $\left[\Lambda^{\prime}, n^{\prime}, r^{\prime}, \gamma\right]$ is a semisimple stratum equivalent to $\left[\Lambda^{\prime}, n^{\prime}, r^{\prime}, \beta\right]$. Put $m_{0}^{\prime}=\max \left\{m^{\prime}, \frac{r^{\prime}}{2}\right\}$ and define $\theta_{i}^{\prime}$ and $\theta_{0}^{\prime}$ as above; note that we have $\left\lfloor\frac{m_{0}^{\prime}}{e\left(\Lambda^{\prime} \mid \mathfrak{o}_{F}\right)}\right\rfloor=\left\lfloor\frac{m_{0}}{e\left(\Lambda \mid \mathfrak{o}_{F}\right)}\right\rfloor$.

Proposition 3.26 (cf. [6] (3.6.1)). There exists a canonical bijection

$$
\tau_{\Lambda, \Lambda^{\prime}, \beta}: \mathcal{C}(\Lambda, m, \beta) \rightarrow \mathcal{C}\left(\Lambda^{\prime}, m^{\prime}, \beta^{\prime}\right)
$$

such that, for $\theta \in \mathcal{C}(\Lambda, m, \beta), \theta^{\prime}:=\tau_{\Lambda, \Lambda^{\prime}, \beta}(\theta)$ is the unique simple character in $\mathcal{C}\left(\Lambda^{\prime}, m^{\prime}, \beta^{\prime}\right)$ such that $B_{\beta}^{\times} \cap I_{\widetilde{G}}\left(\theta, \theta^{\prime}\right) \neq \emptyset$. Moreover $B_{\beta}^{\times} \subset I_{\widetilde{G}}\left(\theta, \theta^{\prime}\right)$.

Proof Let $\theta \in \mathcal{C}(\Lambda, m, \beta)$ be as above and suppose $\theta^{\prime} \in \mathcal{C}\left(\Lambda^{\prime}, m^{\prime}, \beta^{\prime}\right)$ is such that $b \in I_{\widetilde{G}}\left(\theta, \theta^{\prime}\right) \cap B_{\beta}^{\times}$. Then $b_{i} \in I_{\widetilde{G}_{i}}\left(\theta_{i}, \theta_{i}^{\prime}\right) \cap B_{\beta_{i}}^{\times}$so we have $\theta_{i}^{\prime}=\tau_{\Lambda^{i}, \Lambda^{\prime}{ }^{i}, \beta_{i}}\left(\theta_{i}\right)$ by the simple case. But $\theta^{\prime}$ is trivial on $N_{u}$, $N_{l}$ and hence it is clearly uniquely determined.

So we need only show the existence of such a $\theta^{\prime}$. We proceed by induction on $k_{0}(\beta, \Lambda)=-r$, the simple case being given by Lemma 2.6 (see also [6] (3.6.1)). Consider $\tau_{\Lambda, \Lambda^{\prime}, \gamma}\left(\theta_{0}\right) \psi_{\gamma-\beta}$ as a character of $H^{m_{0}^{\prime}+}\left(\beta, \Lambda^{\prime}\right)$. Then, by 2.7) and putting $H=H^{m_{0}+}\left(\beta, \Lambda^{\prime}\right) \cap \widetilde{G}_{i}$ and $H^{\prime}=H^{m_{0}^{\prime}+}\left(\beta, \Lambda^{\prime}\right) \cap \widetilde{G}_{i}$, we have

$$
\begin{aligned}
\left.\tau_{\Lambda^{i}, \Lambda^{\prime i}, \beta_{i}}\left(\theta_{i}\right)\right|_{H^{\prime}}=\tau_{\Lambda^{i}, \Lambda^{\prime i}, \beta_{i}}\left(\left.\theta_{i}\right|_{H}\right) & =\tau_{\Lambda^{i}, \Lambda^{\prime i}, \gamma_{i}}\left(\left.\theta_{0}\right|_{H}\right) \psi_{\gamma_{i}-\beta_{i}} \\
& =\left.\tau_{\Lambda, \Lambda^{\prime}, \gamma}\left(\theta_{0}\right) \psi_{\gamma-\beta}\right|_{H^{\prime}}
\end{aligned}
$$


Then, by Lemma 3.15, there exists $\theta^{\prime} \in \mathcal{C}\left(\Lambda^{\prime}, m^{\prime}, \beta\right)$ such that $\left.\theta^{\prime}\right|_{H^{m_{0}^{\prime}+\left(\beta, \Lambda^{\prime}\right)}}=\tau_{\Lambda, \Lambda^{\prime}, \gamma}\left(\theta_{0}\right) \psi_{\gamma-\beta}$ and $\left.\theta^{\prime}\right|_{\widetilde{G}_{i}}=\tau_{\Lambda^{i}, \Lambda^{\prime}, \beta_{i}}\left(\theta_{i}\right)$. Finally, we have $B_{\beta}^{\times} \subset I_{\widetilde{G}}\left(\theta, \theta^{\prime}\right)$ by Lemma 2.1 .

We remark that the above result holds also without the assumption $r=-k_{0}(\beta, \Lambda)$, since the set $\mathcal{C}(\Lambda, m, \beta)$ does not depend on $r$.

\subsection{Semisimple characters for $G$}

Finally, in this section we describe the situation for the group $G$. As for simple characters, the semisimple characters of $G$ will be obtained by transfer from those for $\widetilde{G}$.

Let $[\Lambda, n, r-, \beta]$ be a semisimple stratum in $A$ which, in addition, is skew - that, is, $\beta \in A_{-}$and the decomposition $V=\bigoplus_{i=1}^{l} V^{i}$ is orthogonal with respect to the form $h$. Let $[\Lambda, n, r, \gamma]$ be a semisimple stratum equivalent to $[\Lambda, n, r, \beta]$, with $\gamma \in M$. Then, by [13] (1.10), we may in fact suppose that $\gamma \in M_{-}$so the stratum is skew. In particular, we see that the groups $H^{m+}(\beta, \Lambda)$, $J^{m+}(\beta, \Lambda), \Gamma_{m}(\beta, \Lambda)$ are fixed by $\Sigma$ and that $\Sigma$ acts on the set $\mathcal{C}(\Lambda, m, \beta)$ of semisimple characters. We put $H_{-}^{m+}(\beta, \Lambda)=H^{m+}(\beta, \Lambda)^{\Sigma}$, and likewise for $J_{-}^{m+}(\beta, \Lambda)$ and $\Gamma_{m}^{-}(\beta, \Lambda)$. Then Glauberman's correspondence $([9]$ - see [13] $\S 2$ for this situation) gives a bijection $\mathbf{g}$ between the irreducible representations of $H^{m+}(\beta, \Lambda)$ fixed by $\Sigma$ and the irreducible representations of $H_{-}^{m+}(\beta, \Lambda)$. We set

$$
\mathcal{C}_{-}(\Lambda, m, \beta)=\left\{\mathbf{g}(\theta): \theta \in \mathcal{C}^{\Sigma}(\Lambda, m, \beta)\right\},
$$

where $\mathcal{C}^{\Sigma}(\Lambda, m, \beta)$ denotes the semisimple characters fixed by $\Sigma$, and $\theta_{-} \in \mathcal{C}_{-}(\Lambda, m, \beta)$ is called a skew semisimple character. Note that, since $\theta$ is a character, $\mathbf{g}(\theta)$ is in fact just the restriction of $\theta$.

Proposition 3.27. Let $\theta_{-} \in \mathcal{C}_{-}(\Lambda, m, \beta)$; then $I_{G}\left(\theta_{-}\right)=\Gamma_{m}^{-}(\beta, \Lambda) \cdot B_{\beta} \cap G \cdot \Gamma_{m}^{-}(\beta, \Lambda)$.

Proof We have $\theta_{-}=\mathbf{g}(\theta)$, for some $\theta \in \mathcal{C}^{\Sigma}(\Lambda, m, \beta)$ and, by [13] $(2.5), I_{G}\left(\theta_{-}\right)=i_{\widetilde{G}}(\theta)^{\Sigma}$. Now the result follows from Theorem 3.22 and [12] (2.3), if we can show that, for $b \in B_{\beta}^{\times}$,

$$
\Gamma_{m} b \Gamma_{m} \cap B_{\beta}^{\times}=\Gamma_{m} \cap B_{\beta}^{\times} \cdot b \cdot \Gamma_{m} \cap B_{\beta}^{\times} .
$$

Note that the containment $\supset$ is clear.

Put $m^{\prime}=\min \left\{r-m, \frac{r}{2}\right\}$; then we have $\mathfrak{b}_{m^{\prime}} \subset \mathfrak{m}_{m} \subset \mathfrak{a}_{m^{\prime}}$ so $\Gamma_{m} \cap B_{\beta}^{\times}=U_{m^{\prime}}(\Lambda) \cap B_{\beta}^{\times}$and $\Gamma_{m} \subset U_{m^{\prime}}(\Lambda)$. Then, by [6] (1.6.1), we have

$$
\Gamma_{m} b \Gamma_{m} \cap B_{\beta}^{\times} \subset U_{m^{\prime}}(\Lambda) b U_{m^{\prime}}(\Lambda) \cap B_{\beta}^{\times}=U_{m^{\prime}}(\Lambda) \cap B_{\beta}^{\times} \cdot b \cdot U_{m^{\prime}}(\Lambda) \cap B_{\beta}^{\times}
$$

as required.

Proposition 3.28. Let $\theta_{-} \in \mathcal{C}_{-}(\Lambda, m-, \beta)$. Then the pairing

$$
\boldsymbol{k}_{\theta_{-}}:\left(g, g^{\prime}\right) \mapsto \theta\left[g, g^{\prime}\right], \quad g, g^{\prime} \in J_{-}^{m}(\beta, \Lambda)
$$

induces a nondegenerate alternating bilinear form

$$
J_{-}^{m}(\beta, \Lambda) / H_{-}^{m}(\beta, \Lambda) \times J_{-}^{m}(\beta, \Lambda) / H_{-}^{m}(\beta, \Lambda) \rightarrow \mathbb{C}^{\times} .
$$


Proof We have $\theta_{-}=\mathbf{g}(\theta)$, for some $\theta \in \mathcal{C}^{\Sigma}(\Lambda, m-, \beta)$ and we consider the form $\boldsymbol{k}_{\theta}: J^{m} / H^{m} \times$ $J^{m} / H^{m} \rightarrow \mathbb{C}^{\times}$. Now $\sigma$ acts linearly on the $k_{F}$-space $J^{m} / H^{m}$ and, moreover, preserves $\boldsymbol{k}_{\theta}$. The result is now immediate from Proposition 3.24 , since the homomorphism $J_{-}^{m} \hookrightarrow J^{m}$ induces an isomorphism $J_{-}^{m} / H_{-}^{m} \simeq\left(J^{m} / H^{m}\right)^{\Sigma}$ and $\boldsymbol{k}_{\theta_{-}}$corresponds to the restriction of $\boldsymbol{k}_{\theta}$ to $\left(J^{m} / H^{m}\right)^{\Sigma}$.

Corollary 3.29. Let $\theta \in \mathcal{C}^{\Sigma}(\Lambda, m, \beta)$ and put $\theta_{-}=\mathbf{g}(\theta) \in \mathcal{C}_{-}(\Lambda, m, \beta)$. Then there exists a unique irreducible representation $\eta_{-}$of $J_{-}^{m+}(\beta, \Lambda)$ which contains $\theta_{-}, \operatorname{dim} \eta_{-}=\left(J_{-}^{m+}: H_{-}^{m+}\right)^{\frac{1}{2}}$ and $I_{G}\left(\eta_{-}\right)=\Gamma_{m}^{-} \cdot B_{\beta} \cap G \cdot \Gamma_{m}^{-}$. Moreover, if $\eta$ is the irreducible representation of $J^{m+}(\beta, \Lambda)$ containing $\theta$, then we have $\eta_{-}=\mathbf{g}(\eta)$.

Proof The first assertions are immediate from the previous proposition and, moreover, $\operatorname{dim} \eta_{-}=$ $\left(J_{-}^{m+}(\beta, \Lambda): H_{-}^{m+}(\beta, \Lambda)\right)^{\frac{1}{2}}$. Now the restriction $\left.\eta\right|_{H_{-}^{m+}(\beta, \Lambda)}$ is a multiple of $\theta_{-}$so $\left.\eta\right|_{J_{-}^{m+}(\beta, \Lambda)}$ is a multiple of $\eta_{-}$. In particular, $\eta_{-}=\mathbf{g}(\eta)$.

Remark 3.30. Let $[\Lambda, n, r-, \beta]$ be a skew semisimple stratum in $A$, with associated splitting $V=\bigoplus_{i=1}^{l} V^{i}$, and put $E_{i}=F\left[\beta_{i}\right]$ as usual; we also denote $E_{i, 0}$ the fixed field of the involution in $E_{i}$. Suppose that we have $\sum_{i=1}^{l}\left[E_{i} ; F\right]=N$; then $J_{-}(\beta, \Lambda) / J_{-}^{1}(\beta, \Lambda) \simeq \prod_{i=1}^{l} N_{1}\left(k_{i}\right)$ is a product of cyclic groups, where $k_{i}$ is the residue field of $E_{i}$ and $N_{1}\left(k_{i}\right)$ denotes the elements $x \in k_{i}$ such that $N_{k_{i} / k_{i, 0}} x=1$, where $k_{i, 0}$ is the residue field of $E_{i, 0}$. In particular, there exists an extension of $\eta_{-}$ to a representation $\kappa_{-}$of $J_{-}(\beta, \Lambda)$, and any extension takes the form $\kappa_{-} \otimes \chi$, for $\chi$ the inflation of a character of $J_{-} / J_{-}^{1}$. We have $I_{G}\left(\kappa_{-}\right) \subset I_{G}\left(\eta_{-}\right)=J_{-}^{1} \cdot B_{\beta} \cap G \cdot J_{-}^{1}=J_{-}$so the induced representation

$$
\pi=\text { Ind }{ }_{J_{-}}^{G} \kappa_{-}
$$

is irreducible and supercuspidal (since $J_{-}$is compact) and $\left(J_{-}, \kappa_{-}\right)$is a $[G, \pi]_{G^{-}}$type.

In general, to obtain a supercuspidal representation we will have to extend $\eta_{-}$to a representation of $J_{-}$and twist by the inflation of a cuspidal representation of $J_{-} / J_{-}^{1}$. In order to control the intertwining of this representation (cf. [6] (5.3), [15] (SC3)), we will need the following result:

Proposition 3.31 (cf. [6] (5.1.8), [13] (4.3)). Let $[\Lambda, n, 0, \beta]$ be a skew semisimple stratum in $A$, let $\theta_{-} \in \mathcal{C}_{-}(\Lambda, 0, \beta)$ and let $\eta_{-}$be as in Corollary 3.29. Then, for $g \in G$, we have

$$
\operatorname{dim} I_{g}\left(\eta_{-}, \eta_{-}\right)= \begin{cases}1 & \text { if } g \in J_{-}^{1} \cdot B_{\beta} \cap G \cdot J_{-}^{1} ; \\ 0 & \text { otherwise. }\end{cases}
$$

Proof As in [6] (5.1.8), the result will follow if we can show that, for $y \in B_{\beta} \cap G, J_{-}^{1} y J_{-}^{1}$ is the union of $\left(J_{-}^{1}: H_{-}^{1}\right)$ distinct $\left(H_{-}^{1}, H_{-}^{1}\right)$-double cosets, where $\left(K_{1}: K_{2}\right)$ denotes group index. As in [6] (5.1.9), it is then enough to prove that $\left(J_{-}^{1}: J_{-}^{1} \cap\left(J_{-}^{1}\right)^{y}\right)=\left(H_{-}^{1}: H_{-}^{1} \cap\left(H_{-}^{1}\right)^{y}\right)$.

Now we have $J_{-}^{1}=C\left(\mathfrak{J}_{-}^{1}\right)$ and $H_{-}^{1}=C\left(\mathfrak{H}_{-}^{1}\right)$, where $C$ denotes the Cayley transform; also $J_{-}^{1} \cap$ $\left(J_{-}^{1}\right)^{y}=C\left(\mathfrak{J}_{-}^{1} \cap\left(\mathfrak{J}_{-}^{1}\right)^{y}\right)$ and likewise for $H_{-}^{1}$. Then, as in [6] (5.1.10), the result will follow if we can show that the following sequence is exact:

$$
0 \rightarrow \mathfrak{b}_{\beta, 1}^{-}+\left(\mathfrak{b}_{\beta, 1}^{-}\right)^{y} \rightarrow \mathfrak{J}_{-}^{1} \cap\left(\mathfrak{J}_{-}^{1}\right)^{y} \stackrel{a_{\beta}}{\rightarrow}\left(\mathfrak{H}_{-}^{1}\right)^{*} \cap\left(\left(\mathfrak{H}_{-}^{1}\right)^{*}\right)^{y} \stackrel{s}{\rightarrow} \mathfrak{b}_{\beta, 0}^{-}+\left(\mathfrak{b}_{\beta, 0}^{-}\right)^{y} \rightarrow 0,
$$

where $s$ is a tame corestriction on $A$ given by

$$
s\left(\sum_{i, j=1}^{l} a_{i j}\right)=\sum_{i=1}^{l} s_{i}\left(a_{i i}\right), \quad a_{i j} \in A^{i j},
$$


for $s_{i}$ a tame corestriction on $A^{i i}$ relative to $E_{i} / F$ which commutes with the involution, $1 \leq i \leq l$. Exactness comes from Lemma 3.17 at all places except the final one, where it follows from the simple case [7] (6.3) Lemma.

We end this section by looking at the transfer of skew semisimple characters.

Proposition 3.32. Let $[\Lambda, n, 0, \beta],\left[\Lambda^{\prime}, n^{\prime}, 0, \beta\right]$ be skew semisimple strata in $A$ and let $m, m^{\prime}$ be such that $0 \leq m<k_{0}(\beta, \Lambda)$ and $\left\lfloor\frac{m^{\prime}}{e\left(\Lambda^{\prime} \mid \mathfrak{o}_{F}\right)}\right\rfloor=\left\lfloor\frac{m}{e\left(\Lambda||_{F}\right)}\right\rfloor$. Then the bijection $\tau_{\Lambda, \Lambda^{\prime}, \beta}$ given by Proposition 3.26 commutes with $\sigma$.

Proof Given $\theta \in \mathcal{C}(\Lambda, m, \beta)$ with $\left.\theta\right|_{\widetilde{G}_{i}}=\theta_{i} \in \mathcal{C}\left(\Lambda^{i}, m, \beta_{i}\right)$, the transfer $\theta^{\prime}:=\tau_{\Lambda, \Lambda^{\prime}, \beta}(\theta)$ is the character of $H^{m^{\prime}+}\left(\beta, \Lambda^{\prime}\right)$ which is trivial on $N_{u}, N_{l}$ and such that $\left.\theta^{\prime}\right|_{\widetilde{G}_{i}}=\tau_{\Lambda^{i}, \Lambda^{\prime i}, \beta_{i}}\left(\theta_{i}\right)$ so the result follows immediately from the simple case in Proposition 2.12 .

In particular, this implies that we have a canonical bijection

$$
\tau_{\Lambda, \Lambda^{\prime}, \beta}: \mathcal{C}_{-}(\Lambda, m, \beta) \rightarrow \mathcal{C}_{-}\left(\Lambda^{\prime}, m^{\prime}, \beta\right)
$$

and, for $\theta_{-} \in \mathcal{C}_{-}(\Lambda, m, \beta), \theta_{-}^{\prime}:=\tau_{\Lambda, \Lambda^{\prime}, \beta}\left(\theta_{-}\right)$is the unique simple character such that $1 \in I_{G}\left(\theta_{-}, \theta_{-}^{\prime}\right)$, by [13] (2.5).

\section{Relatively G-split strata}

In this section we look at the "relatively $G$-split" case and construct a non-zero Jacquet module (cf. [7] $\S 6$ and [14] 33 ). This will be crucial to the refinement process in $\$ 5$.

\subsection{Definition and intertwining}

Let $[\Lambda, n, m, \beta]$ be a skew semisimple stratum in $A$, with associated splitting $V=\bigoplus_{i=1}^{l} V^{i}$. In this section we will assume that $m$ is an integer. We have $B_{\beta}$ the $A$-centralizer of $\beta$ and let $B_{i}$ be the $A^{i i}$-centralizer of $\beta_{i}, 1 \leq i \leq l$, so that $B_{\beta}=\bigoplus_{i=1}^{l} B_{i}$. We put $E_{i}=F\left[\beta_{i}\right]$ and $\mathfrak{o}_{i}=\mathfrak{o}_{E_{i}}$, for $1 \leq i \leq l$, and let $s_{i}$ be a tame corestriction on $A^{i i}$ relative to $E_{i} / F$. We suppose we are given a decomposition of $E_{1}$-vector spaces $V^{1}=V_{0}^{1} \perp\left(V_{1}^{1} \oplus V_{-1}^{1}\right)$, where $V_{1}^{1}$ and $V_{-1}^{1}$ are totally isotropic with respect to $h$, such that $\Lambda^{1}(k)=\bigoplus_{-1 \leq j \leq 1}\left(\Lambda^{1}(k) \cap V_{j}^{1}\right)$. We will write $A^{11}=\bigoplus_{-1 \leq j, k \leq 1} A_{j k}^{11}$ for the corresponding decomposition of $A^{11}$.

Let $b_{i} \in A^{i i} \cap \mathfrak{a}_{-m}$, for $1 \leq i \leq l$, where $b_{1}=\sum_{-1 \leq j \leq 1} b_{1, j}$ with $b_{1, j} \in A_{j j}^{11}$. We suppose that the derived stratum $\left[\Lambda_{\mathfrak{o}_{1}}^{1}, m, m-1, s_{1}\left(b_{1}\right)\right]$ in $B_{1}$ is $G$-split (see Definition 1.3 ) by the decomposition $V^{1}=V_{0}^{1} \perp\left(V_{1}^{1} \oplus V_{-1}^{1}\right)$ (cf. [14] $\S 3.1$ ). That is, the stratum is split by this decomposition, the stratum $\left[\Lambda_{\mathfrak{o}_{1}}^{1,1}, m, m-1, s_{1}\left(b_{1,1}\right)\right]$ has characteristic polynomial of the form $\psi(X)^{d}$ and the stratum $\left[\Lambda_{\mathfrak{o}_{1}}^{1,-1}, m, m-1, s_{1}\left(b_{1,-1}\right)\right]$ has characteristic polynomial $\bar{\psi}(\eta X)^{d}$, where $\eta=(-1)^{m / g}(-1)^{e / g}$ with $e=e\left(\Lambda^{1} \mid \mathfrak{o}_{1}\right)$ and $g=(m, e)$; in particular, $\psi(X)$ is coprime to $\bar{\psi}(\eta X)$.

We now consider the decomposition

$$
V=V_{-1}^{1} \oplus\left(V_{0}^{1} \oplus \bigoplus_{i=1}^{l} V^{i}\right) \oplus V_{1}^{1}
$$


and we will abbreviate $V_{-1}^{1}=V_{-1}, V_{1}^{1}=V_{1}$ and $V_{0}^{1} \oplus \bigoplus_{i=1}^{l} V^{i}=V_{0}$. We will consider the block picture $A=\bigoplus_{-1 \leq j, k \leq 1} A_{j k}$ with respect to this splitting and we put $A_{u}=\bigoplus_{-1 \leq j<k \leq 1} A_{j k}$, $A_{l}=\bigoplus_{-1 \leq k<j \leq 1} A_{j k}$, as usual. We also put $\mathcal{M}=\bigoplus_{-1 \leq j \leq 1} A_{j j}, M=\mathcal{M}^{\times}, N_{u}=1+A_{u}$, $N_{l}=1+A_{l}, P_{u}=M N_{u}, P_{l}=M N_{l}$, and we write $b_{0}=b_{1,0}+\sum_{i=2}^{l} b_{i} \in A_{0,0}$. We will also retain the block notation $A^{i i}=\operatorname{End}_{F} V^{i}$, for $1 \leq i \leq l$, alongside this new block decomposition.

We look at the lattice

$$
\mathfrak{k}=\left(\begin{array}{ccc}
\mathfrak{H}^{m} & \mathfrak{b}_{1}+\mathfrak{m}_{m-1} & \mathfrak{b}_{1}+\mathfrak{m}_{m-1} \\
\mathfrak{H}^{m} & \mathfrak{H}^{m} & \mathfrak{b}_{1}+\mathfrak{m}_{m-1} \\
\mathfrak{H}^{m} & \mathfrak{H}^{m} & \mathfrak{H}^{m}
\end{array}\right),
$$

where $\mathfrak{b}_{1}=\mathfrak{b}_{\beta, 1}=\mathfrak{a}_{1} \cap B_{\beta}$ and $\mathfrak{m}_{m-1}=\mathfrak{m}_{m-1}(\beta, \Lambda)$. Since $\Gamma_{m-1}=1+\mathfrak{m}_{m-1}$ and $1+\mathfrak{b}_{1}$ normalize $H^{m}(\beta, \Lambda), K=1+\mathfrak{k}$ is a compact open subgroup of $\widetilde{G}$ which is, moreover, fixed by $\Sigma$.

Proposition 4.1 (cf. [7] (6.1)). Let $\theta \in \mathcal{C}^{\Sigma}(\Lambda, m-1, \beta)$. There exists a unique character $\vartheta$ of $K$ which extends $\theta$ and is trivial on $K \cap N_{u}$. Moreover, $\vartheta$ is fixed by $\sigma$.

Proof Uniqueness is clear, while existence is because $K \cap N_{u}$ normalizes $\theta$. The final statement is clear, since $K \cap N_{u}$ is fixed by $\sigma$.

Note that, if $0 \leq k \leq m-1$ and $\widetilde{\theta} \in \mathcal{C}(\Lambda, k, \beta)$ extends $\theta$, then $\widetilde{\theta}$ is trivial on $H^{k+1} \cap N_{u}$ (and on $H^{k+1} \cap N_{l}$ ), by [7] (5.2) Proposition and Lemma 3.15(i), so $\vartheta$ and $\widetilde{\theta}$ agree on $H^{k+1} \cap K$.

We now consider the character $\xi=\vartheta \psi_{b}$ of the group $K$, where $b=b_{-1,1}+b_{0}+b_{1,1}$ and $\psi_{b}$ denotes the extension of $\left.\psi_{b}\right|_{H^{m}}$ to $K$ which is trivial on $K \cap N_{u}$.

Theorem 4.2 (cf. [7] (6.2)). Suppose $g \in N_{u}$ intertwines the character $\xi$ of $K$. Then $g \in K \cap N_{u}$.

Before giving the proof of this theorem, we observe the following easy consequence:

Corollary 4.3. Let $\xi_{-}$be the restriction of $\xi$ to the group $K_{-}=K \cap G$. Suppose $g \in N_{u}^{-}$intertwines $\xi_{-} ;$then $g \in K_{-} \cap N_{u}^{-}$.

Proof We have $I_{G}\left(\xi_{-}\right)=I_{\widetilde{G}}(\xi) \cap G$, by [13] (2.5), so the result is immediate.

Now we will prove Theorem 4.2 so we suppose $g \in I_{\widetilde{G}}(\xi \mid K)$. Then $g$ certainly intertwines $\left.\xi\right|_{H^{m+1}}=$ $\left.\theta\right|_{H^{m+1}} \in \mathcal{C}(\Lambda, m, \beta)$ so we have $g \in \Gamma_{m} B_{\beta}^{\times} \Gamma_{m}$. We will first show

$$
g \in \Gamma M \Gamma,
$$

where $\Gamma=1+\mathfrak{b}_{1}+\mathfrak{m}_{m-1}$. Note also that $\Gamma$ normalizes the pair $\left(H^{m},\left.\xi\right|_{H^{m}}\right)$.

We write $g=(1+x) t(1+y)^{-1}$, with $x, y \in \mathfrak{m}_{m}$, and $t \in B_{\beta}^{\times}$. By Lemma 3.21, we have

$$
\theta^{1+x}=\theta \psi_{(1+x)^{-1} \beta(1+x)-\beta}
$$

as characters of $H^{m}(\beta, \Lambda)$. But

$$
(1+x)^{-1} \beta(1+x)-\beta=a_{\beta}(x)-(1+x)^{-1} x a_{\beta}(x)
$$


and $(1+x)^{-1} x a_{\beta}(x) \in\left(\mathfrak{H}^{m+1}\right)^{*}$, by Lemma 3.17 and Lemmas 3.10 , 3.11, so we have

$$
\theta^{1+x}=\theta \psi_{a_{\beta}(x)} .
$$

Now $t$ intertwines the restrictions of $\xi^{1+x}, \xi^{1+y}$ to $H^{m}(\beta, \Lambda)$ so it intertwines their restrictions to $H^{m}(\beta, \Lambda) \cap B_{\beta}^{\times}$. Now $\psi_{a_{\beta}(x)}, \psi_{a_{\beta}(y)}$ restrict trivially here, whilst $(1+x),(1+y)$ fix the characters $\psi_{b}$, so $t$ intertwines $\theta \psi_{b}$ on $H^{m}(\beta, \Lambda) \cap B_{\beta}^{\times}$. But $\theta \in \mathcal{C}(\Lambda, m-1, \beta)$ so $\left.\theta\right|_{H^{m}(\beta, \Lambda) \cap B_{\beta}^{\times}}$is intertwined by all of $B_{\beta}^{\times}$, by Theorem 3.22 and $t$ intertwines the character $\left.\psi_{b}\right|_{H^{m}(\beta, \Lambda) \cap B_{\beta}^{\times}}$.

We write $t=\sum_{i=1}^{l} t_{i}$, with $t_{i} \in B_{i}^{\times}$, for $1 \leq i \leq l$, and look at the character $\left.\psi_{b}\right|_{H^{m}(\beta, \Lambda) \cap B_{1}^{\times}}=\psi_{b_{1}}$. There exists a character $\psi_{B_{1}}$ of $B_{1}$, of the form $\psi_{E_{1}} \circ \operatorname{tr}_{B_{1} / E_{1}}$, such that $\left.\psi_{a}\right|_{B_{1}}=\psi_{B_{1}, s_{1}(a)}$ for any $a \in A^{11}$. In particular, $\left.\psi_{b_{1}}\right|_{B_{1}}=\psi_{B_{1}, s_{1}\left(b_{1}\right)}$ so [12] (4.14) shows that

$$
t_{1} \in U_{1}\left(\Lambda_{\mathfrak{o}_{1}}^{1}\right) \cdot B_{1}^{\times} \cap M \cdot U_{1}\left(\Lambda_{\mathfrak{o}_{1}}^{1}\right) .
$$

Then, since $B_{i}^{\times} \subset M$ for $2 \leq i \leq l$, we have

$$
t \in\left(1+\mathfrak{b}_{1}\right) \cdot B_{\beta}^{\times} \cap M \cdot\left(1+\mathfrak{b}_{1}\right) .
$$

As $\left(1+\mathfrak{b}_{1}\right)$ normalizes $\Gamma_{m}$, we may absorb these factors into $\Gamma$ and assume $g=(1+x) t(1+y)$ with $x, y \in \mathfrak{m}_{m}$ and $t \in \mathfrak{b}_{\beta}^{\times} \cap M$. We write $1+x=\left(1+x_{l}\right)\left(1+x_{m}\right)\left(1+x_{u}\right)$, with $x_{l} \in A_{l}, x_{m} \in \mathcal{M}$, $x_{u} \in A_{u}$, and, likewise, $1+y=\left(1+y_{u}\right)\left(1+y_{m}\right)\left(1+y_{l}\right)$.

We now consider the restriction of $\xi$ to the group

$$
K_{l}=1+\mathfrak{k}_{l}, \quad \mathfrak{k}_{l}=\left(\begin{array}{ccc}
\mathfrak{H}^{m+1} & \mathfrak{H}^{m} & \mathfrak{H}^{m} \\
\mathfrak{H}^{m+1} & \mathfrak{H}^{m+1} & \mathfrak{H}^{m} \\
\mathfrak{H}^{m+1} & \mathfrak{H}^{m+1} & \mathfrak{H}^{m+1}
\end{array}\right) .
$$

Then, as in the proof of Theorem 3.22, we find that we may assume $x_{l}, y_{l} \in \mathfrak{m}_{m-1}$. (Note that the projections $\mathbf{1}_{i}: V \rightarrow V_{i}$ lie in $B_{\beta}$ so the exact sequences of Lemma 3.17 are still exact.) Repeating with $K_{u}$, again as in the proof of Theorem 3.22 , we get $x_{u}, y_{u} \in \mathfrak{m}_{m-1}$ also so, absorbing these terms into $\Gamma$, we get $g \in \Gamma_{m} \cap M \cdot B_{\beta}^{\times} \cap M \cdot \Gamma_{m} \cap M \subset M$ as required.

So we have proved (4.4). Now let $g \in N_{u}$ intertwine $\xi$; then $g=\gamma m \gamma^{\prime}$, for some $m \in M, \gamma, \gamma^{\prime} \in \Gamma$. The group $\Gamma$ has Iwahori decomposition $\Gamma=\Gamma \cap N_{l} \cdot \Gamma \cap M \cdot \Gamma \cap N_{u}$ so we can write $\gamma=\gamma_{u} \gamma_{M} \gamma_{l}$, $\gamma^{\prime}=\gamma_{l}^{\prime} \gamma_{M}^{\prime} \gamma_{u}^{\prime}$. Thus, for a certain $m^{\prime} \in M$, we have $\gamma_{u}^{-1} g \gamma_{u}^{\prime-1}=\gamma_{l} m^{\prime} \gamma_{l}^{\prime} \in N_{u} \cap P_{l}=\{1\}$. Hence $g=\gamma_{u} \gamma_{u}^{\prime} \in \Gamma \cap N_{u}=K \cap N_{u}$ as required.

\subsection{Covers}

For $-1 \leq i \leq 1$, we put $G_{i}=G \cap \operatorname{Aut}_{F}\left(V^{i}\right)$ and suppose that we are given:

(i) a subgroup $K_{1}$ of $U\left(\Lambda^{1}\right)$ containing and normalizing $H_{-}^{m} \cap G_{1}$ and an irreducible representation $\rho_{1}$ of $K_{1}$ whose restriction to $K_{1} \cap K_{-}$is a multiple of $\xi_{-}$;

(ii) a subgroup $K_{0}^{-}$of $P\left(\Lambda^{0}\right)$ containing and normalizing $H_{-}^{m} \cap G_{0}$ and an irreducible representation $\rho_{0}^{-}$of $K_{0}^{-}$whose restriction to $K_{0}^{-} \cap K_{-}$is a multiple of $\xi_{-}$.

We think of $K_{1}$ embedded in $G$ as $\left\{\left(\begin{array}{ccc}k^{\sigma} & & \\ & 1 & \\ & & k\end{array}\right): k \in K_{1}\right\}$. 
Proposition 4.5 (cf. [7] (6.6)). (i) The set $\widetilde{K}_{-}=\left(K_{1} \times K_{0}^{-}\right) . K_{-}$is a group.

(ii) There is a unique irreducible representation $\rho_{-}$of $\widetilde{K}_{-}$which is trivial on $K_{-} \cap N_{u}^{-}, K_{-} \cap N_{l}^{-}$ and whose restriction to $K_{1} \times K_{0}^{-}$is $\rho_{1} \otimes \rho_{0}^{-}$.

(iii) The pair $\left(\widetilde{K}_{-}, \rho_{-}\right)$is a G-cover of $\left(\widetilde{K}_{-} \cap M, \rho_{1} \otimes \rho_{0}^{-}\right)$.

Proof This is identical to [7] (6.6), except we take the element $\zeta$ to be

$$
\zeta=\left(\begin{array}{ccc}
\varpi_{F} & & \\
& 1 & \\
& & \varpi_{F}^{\sigma}
\end{array}\right)
$$

\subsection{Jacquet modules}

Proposition 4.6. Let $(\pi, \mathcal{V})$ be a smooth representation of $G$ which contains $\theta_{-} \psi_{b}^{-}$on $H_{-}^{m}$. Then $\pi$ contains the character $\xi_{-}$of $K_{-}$also.

Proof We first suppose $m \geq 2$ and put, for $q \in \mathbb{Z}$,

$$
\mathfrak{k}_{q}= \begin{cases}\mathfrak{H}^{m}+\mathfrak{H}^{q+1} \cap A_{u} & \text { for }\left\lfloor\frac{m+1}{2}\right\rfloor \leq q \leq m-1 \\ \mathfrak{H}^{m}+\mathfrak{m}_{m-(q+1)} \cap A_{u} & \text { for } 0 \leq q \leq\left\lfloor\frac{m}{2}\right\rfloor-1 ; \\ \mathfrak{H}^{m}+\left(\mathfrak{b}_{1}+\mathfrak{m}_{m-1}\right) \cap A_{u} & \text { for } q=-1\end{cases}
$$

Then we put $K_{q}=1+\mathfrak{k}_{q}$ and $K_{q}^{-}=K_{q} \cap G$. Note, in particular, that $K_{-1}=K$.

We also put

$$
\Xi_{q}^{-}= \begin{cases}\Gamma_{q}^{-} \cap N_{l} & \text { for }\left\lfloor\frac{m+1}{2}\right\rfloor \leq q \leq m-1 \\ H_{-}^{m} \cap N_{l} & \text { for } 1 \leq q \leq\left\lfloor\frac{m}{2}\right\rfloor-1 \\ \left(1+\mathfrak{b}_{m-1}\right) \cap N_{l}^{-} & \text {for } q=0\end{cases}
$$

Lemma 4.7. For $\left\lfloor\frac{m+1}{2}\right\rfloor<q \leq m-1$ or $0 \leq q \leq\left\lfloor\frac{m}{2}\right\rfloor-1, \Xi_{q}^{-}$acts transitively on the characters of $K_{q-1}^{-}$which restrict to $\xi_{-}$on $K_{q}^{-}$.

Proof We treat first the case $\left\lfloor\frac{m+1}{2}\right\rfloor<q \leq m-1$. The quotient $K_{q-1}^{-} / K_{q}^{-}$is abelian so any character of $K_{q-1}^{-}$which restricts to $\xi_{-}$on $K_{q}^{-}$is given by $\xi_{-} \psi_{c}^{-}$, for some $c \in \mathfrak{k}_{q,-}^{*} / \mathfrak{k}_{q-1,-}^{*}$, that is, in $\left(\mathfrak{H}^{q+1}\right)^{*} \cap A_{l}^{-} /\left(\mathfrak{H}^{q}\right)^{*} \cap A_{l}^{-}$.

We have $\mathfrak{m}_{q} \mathfrak{H}^{q} \subset \mathfrak{H}^{m+1}$, by Proposition 2.12 so $\left(\psi_{b}^{-}\right)^{C(x)}=\psi_{b}^{-}$, for $x \in \mathfrak{m}_{q} \cap A_{l}^{-}$, and, for $k \in K_{q-1}^{-}$, we have $[C(x), k] \in H_{-}^{m+1}$. Let $\widetilde{\theta}_{-} \in \mathcal{C}_{-}(\Lambda, q, \beta)$ extend $\theta_{-}$; then, by Lemma 3.21

$$
\theta_{-}[C(x), k]=\widetilde{\theta}_{-}^{C(x)}(k) \widetilde{\theta}_{-}\left(k^{-1}\right)=\psi_{C(x)^{-1} \beta C(x)-\beta}^{-}(k) .
$$

Now $C(x)^{-1} \beta C(x)-\beta \equiv a_{\beta}(x)\left(\bmod \left(\mathfrak{H}^{q}\right)^{*} \cap A_{l}^{-}\right)$so, altogether, we have

$$
\xi_{-}^{C(x)}=\xi_{-} \psi_{a_{\beta}(x)}^{-} .
$$

But, by Lemma 3.17, $a_{\beta}\left(\mathfrak{m}_{q} \cap A_{l}^{-}\right)=\left(\mathfrak{H}^{q+1}\right)^{*} \cap A_{l}^{-}$and the result follows. 
For $1 \leq q \leq\left\lfloor\frac{m}{2}\right\rfloor-1$, the proof is almost identical, with the roles of $\mathfrak{m}$ and $\mathfrak{H}$ reversed.

Suppose then that $q=0$. We have $K_{-1}=K_{0} \cdot\left(1+\mathfrak{b}_{1}\right) \cap N_{u}^{-}$, with the second factor normalizing the first and normalizing $\xi_{-}$on it. Hence any character of $K_{-1}$ which restricts to $\xi_{-}$on $K_{0}$ takes the form $\xi_{-} \psi_{c}^{-}$, for $\psi_{c}^{-}$some character of $\left(1+\mathfrak{b}_{1}\right) \cap N_{u}^{-}$trivial on $\left(1+\mathfrak{b}_{2}\right) \cap N_{u}^{-}$, that is, $c \in \mathfrak{b}_{-1} \cap A_{l}^{-} / \mathfrak{b}_{0} \cap A_{l}^{-}$. Now $\left(1+\mathfrak{b}_{m-1}\right) \cap N_{l}^{-}$normalizes $\xi_{-}$on $K_{0}^{-}$while, on $\left(1+\mathfrak{b}_{1}\right) \cap N_{u}^{-}$, we have

$$
\xi_{-}=\psi_{b}^{-}=\psi_{B_{1}, s_{1}\left(b_{1}\right)}^{-},
$$

where $s_{1}$ is a tame corestriction on $A^{11}$ relative to $E_{1} / F$ which commutes with the involution. (Note that $B_{\beta} \cap A_{u} \subset B_{1}$ and, likewise $B_{\beta} \cap A_{l} \subset B_{1}$.) We put $\delta=s_{1}\left(b_{1}\right) \in \mathfrak{b}_{-m}^{-}$. Then for $x \in \mathfrak{b}_{m-1} \cap A_{l}^{-}$, we have

$$
\left(\psi_{B_{1}, \delta}^{-}\right)^{C(x)}=\psi_{B_{1}, \delta}^{-} \psi_{B_{1}, a_{\delta}(x)}^{-}
$$

Then the result follows since $a_{\delta}\left(\mathfrak{b}_{m-1} \cap A_{l}^{-}\right)=\mathfrak{b}_{1} \cap A_{l}^{-}$as in [14] (3.4).

Lemma 4.8. $\Xi_{\left\lfloor\frac{m+1}{2}\right\rfloor}^{-}$acts transitively on the characters of $K_{\left\lfloor\frac{m}{2}\right\rfloor-1}^{-}$which restrict to $\xi$ on $K_{\left\lfloor\frac{m+1}{2}\right\rfloor}^{-}$.

Proof The proof is almost identical to the first case of the previous lemma, but we must use Lemma 3.23 in place of Lemma 3.21 .

The proof of Proposition 4.6 in the case $m \geq 2$ is now immediate since, by successively conjugating by a suitable element of $\Xi_{q}^{-}$, we see that $\pi$ contains $\xi_{-} \mid K_{q-1}^{-}$(where $q-1$ should be replaced by $\left\lfloor\frac{m}{2}\right\rfloor-1$ in the case $q=\left\lfloor\frac{m+1}{2}\right\rfloor$ ). The proof in the case $m=1$ is similar but easier, requiring only Lemma 4.8

Theorem 4.9. Let $(\pi, \mathcal{V})$ be a smooth representation of $G$ containing the character $\theta_{-} \psi_{b}^{-}$of $H_{-}^{m}$. Then $\pi$ is not supercuspidal.

Proof By Proposition 4.6, $(\pi, \mathcal{V})$ contains the character $\xi_{-} \mid K_{-}$and, by Proposition 4.5, the pair $\left(K_{-}, \xi_{-}\right)$is a $G$-cover of $\left(K_{-} \cap M, \xi_{-} \mid K_{-} \cap M\right)$. Then, by [8] (7.9), the $\left.\xi_{-}\right|_{K_{-}} \cap M_{\text {-isotypic component }}$ $\mathcal{V}_{u}^{\left.\xi_{-}\right|_{K_{-} \cap M}}$ of the Jacquet module of $\mathcal{V}$ with respect to $P_{u} \cap G$ is non-zero.

\section{Supercuspidal Representations}

This section is devoted to the proof of our main theorem, that every positive-level irreducible supercuspidal representation of $G$ contains a skew semisimple character. The proof is very much along the lines of [6] (8.1.5), though there are added geometric complications. In particular, our Lemma 5.4 (whose proof is given in $\$ 5.2$ would be relatively straightforward in the case of $\widetilde{G}$.

\subsection{The main theorem}

Theorem 5.1 (cf. [6] (8.1)). Let $\pi$ be a positive-level irreducible supercuspidal representation of $G$. Then $\pi$ contains a skew semisimple character $\theta_{-} \in \mathcal{C}_{-}(\Lambda, 0, \beta)$, for some skew semisimple stratum $[\Lambda, n, 0, \beta]$. 
Proof We know from [14 $\S 2.3$ that $\pi$ contains some skew semisimple stratum $[\Lambda, n, n-1, \beta]$ in $A$. We consider pairs $\left([\Lambda, n, m, \beta], \theta_{-}\right)$consisting of a skew semisimple stratum with $m \in \mathbb{Z}$ and a semisimple character $\theta_{-} \in \mathcal{C}_{-}(\Lambda, m, \beta)$ such that $\pi \mid H_{-}^{m+1}(\beta, \Lambda)$ contains $\theta_{-}$.

If $m=0$ then we are done, so assume $m \geq 1$ for all such pairs. Then $\pi$ contains some irreducible representation $\vartheta$ of $H_{-}^{m}(\beta, \Lambda)$ such that $\vartheta \mid H_{-}^{m+}(\beta, \Lambda)$ contains $\theta_{-}$. However, $\theta_{-}$extends to an abelian character $\widetilde{\theta}_{-} \in \mathcal{C}_{-}(\Lambda, m-1, \beta)$ of $H_{-}^{m}(\beta, \Lambda)$ and the quotient $H_{-}^{m} / H_{-}^{m+}$ is abelian. Hence $\vartheta$ is one-dimensional and we may write

$$
\vartheta=\tilde{\theta}_{-} \psi_{c}^{-} \mid H_{-}^{m}(\beta, \Lambda) \quad \text { for some } c \in \mathfrak{a}_{-m}^{-} .
$$

Lemma 5.2 (cf. [6] (8.1.12)). Let $x \in \mathfrak{m}_{m}^{-}$. Then, $C(x)$ normalizes $H_{-}^{m}(\beta, \Lambda)$ and

$$
\vartheta^{C(x)}=\vartheta \psi_{a_{\beta}(x)}^{-} \text {. }
$$

Proof Since $m<r$, we have $\mathfrak{m}_{m} \subset \alpha_{1}$; as $c \in \mathfrak{a}_{m}$, we surely have $\psi_{c}^{C(x)}=\psi_{c}$ on $H^{m}$. On the other hand, by Lemma 3.21, we have

$$
\widetilde{\theta}_{-}^{C(x)}=\widetilde{\theta}_{-} \psi_{C(x)^{-1} \beta C(x)-\beta}^{-} ;
$$

a simple computation now shows that

$$
C(x)^{-1} \beta C(x)-\beta \equiv a_{\beta}(x) \quad\left(\bmod \left(\mathfrak{H}^{m+}\right)^{*}\right),
$$

and the lemma follows.

We return to the proof of Theorem 5.1. Let $V=\bigoplus_{i=1}^{l} V^{i}$ be the decomposition associated to the skew semisimple stratum $[\Lambda, n, m, \beta]$. For $i \neq j$, we have $c_{i j} \in \mathfrak{a}_{-m}^{-} \cap A^{i j}$. Hence, by (3.8), there exists $x \in \mathfrak{n}_{-m} \cap \mathfrak{a}_{r-m}^{-}$such that $c_{i j}=-a_{\beta}(x)_{i j}$, for $i \neq j$. By Lemma 5.2 , $\vartheta^{C(x)}=\theta_{-} \psi_{c^{\prime}}^{-}$; this character certainly occurs in $\pi$ and we have $s\left(c^{\prime}\right)=s(c)$ and $c_{i j}^{\prime}=0$, for $i \neq j$. Hence we may, and will, assume that $c \in \mathcal{M}$.

Put $E_{i}=F\left[\beta_{i}\right], \mathfrak{o}_{i}=\mathfrak{o}_{E_{i}}$, and let $s_{i}$ be a tame corestriction on $A^{i i}$ relative to $E_{i} / F$. We consider the derived stratum

$$
\bigoplus_{i=1}^{l}\left[\Lambda_{\mathfrak{o}_{i}}^{(i)}, m, m-1, s_{i}\left(c_{i}\right)\right] .
$$

We will write $E=\oplus_{i=1}^{l} E_{i}, \mathfrak{o}_{E}=\oplus_{i=1}^{l} \mathfrak{o}_{i}$ and we will abuse notation by calling an $\mathfrak{o}_{F}$-lattice sequence $\Lambda^{\prime}$ in $V$ an $\mathfrak{o}_{E}$-lattice sequence if it is of the form $\Lambda^{\prime}=\oplus_{i=1}^{l} \Lambda^{\prime(i)}$, with $\Lambda^{\prime(i)}$ an $\mathfrak{o}_{i}$-lattice sequence in $V^{i}$. We also let $s: \mathcal{M} \rightarrow B$ be the map $s=\oplus_{i=1}^{l} s_{i}$ and we will call it a tame corestriction on $A$ relative to $E / F$. Altogether, we will write the derived stratum above as

$$
\left[\Lambda_{\mathfrak{o}_{E}}, m, m-1, s(c)\right] \text {. }
$$

The following lemma, whose proof we defer to 5.2 will be crucial.

Lemma 5.4. Let $\widetilde{\theta} \in \mathcal{C}_{-}(\Lambda, m-1, \beta)$ and $c \in \mathfrak{a}_{-m}^{-} \cap \mathcal{M}$ be such that $\pi$ contains $\vartheta:=\widetilde{\theta}_{-} \psi_{c}^{-}$on $H_{-}^{m}(\beta, \Lambda)$. Suppose we have an $\mathfrak{o}_{E}$-lattice sequence $\Lambda^{\prime}$, an integer $m^{\prime}$ and $\alpha^{\prime} \in \mathfrak{b}_{-m}^{-}$such that

$$
s(c)+\mathfrak{b}_{-m+} \subset \alpha^{\prime}+\mathfrak{b}_{-m^{\prime}+}^{\prime}
$$

Then there exist $\widetilde{\theta^{\prime}} \in \mathcal{C}_{-}\left(\Lambda^{\prime}, m^{\prime}-1, \beta\right)$ and $c^{\prime} \in \mathfrak{a}_{-m^{\prime}}^{--} \cap \mathcal{M}$ such that $s\left(c^{\prime}\right)=\alpha^{\prime}$ and $\pi$ contains the character $\vartheta^{\prime}:=\theta_{-}^{\prime} \psi_{c^{\prime}}^{-}$of $H_{-}^{m^{\prime}}\left(\beta, \Lambda^{\prime}\right)$. Moreover, if $\alpha^{\prime}=0$ then we may take $c^{\prime}=0$. 
Returning to the proof of Theorem 5.1, we first show that we may assume that the derived stratum in (5.3) is fundamental, in the sense that at least one of the strata $\left[\Lambda_{\mathfrak{o}_{i}}^{(i)}, m, m-1, s_{i}\left(c_{i}\right)\right]$ is fundamental.

Proposition 5.5. Let $[\Lambda, n, m, \beta]$ be a semisimple skew stratum, $\widetilde{\theta}_{-} \in \mathcal{C}_{-}(\Lambda, m-1, \beta)$ and $c \in$ $\mathfrak{a}_{-m}^{-} \cap \mathcal{M}$ be such that $\pi$ contains $\widetilde{\theta}_{-} \psi_{c}^{-}$. Then there exist $\left[\Lambda^{\prime}, n^{\prime}, m^{\prime}, \beta\right]$, a skew semisimple $\mathfrak{o}_{E-}$ stratum, $\widetilde{\theta}_{-}^{\prime} \in \mathcal{C}_{-}\left(\Lambda^{\prime}, m^{\prime}-1, \beta\right)$ and $c \in \mathfrak{a}_{-m^{\prime}}{ }^{-} \cap \mathcal{M}$ such that $m^{\prime} / e\left(\Lambda^{\prime} \mid \mathfrak{o}_{F}\right) \leq m / e\left(\Lambda \mid \mathfrak{o}_{F}\right)$ and $\pi$ contains $\widetilde{\theta}_{-}^{\prime} \psi_{c}^{-}$and the derived stratum $\left[\Lambda_{\mathfrak{o}_{E}}^{\prime}, m^{\prime}, m^{\prime}-1, s(c)\right]$ is fundamental. In particular, $e^{\prime} /\left(m^{\prime}, e^{\prime}\right) \leq N$, where $e^{\prime}=e\left(\Lambda^{\prime} \mid \mathfrak{o}_{F}\right)$.

Proof We first observe that the final assertion follows from the fact that one $\left[\Lambda_{\mathfrak{o}_{i}}^{\prime(i)}, m^{\prime}, m^{\prime}-1, s_{i}\left(c_{i}\right)\right]$ is fundamental (cf. [14] (2.11)).

As above, $\pi$ contains the character $\vartheta=\widetilde{\theta}_{-} \psi_{c}^{-}$, where $\widetilde{\theta}_{-} \in \mathcal{C}_{-}(\Lambda, m-1, \beta)$ extends $\theta_{-}$and $c \in \mathfrak{a}_{-m}^{-}$. If $\left[\Lambda_{\mathfrak{o}_{E}}, m, m-1, s(c)\right]$ is fundamental, we are done so we assume it is not. Then, by [14] (4.3), for $i=1, \ldots, l$, there exist a self-dual $\mathfrak{o}_{i}$-lattice sequence $\Lambda_{\mathfrak{o}_{i}}^{\prime(i)}$, with $e\left(\Lambda^{\prime(i)} \mid \mathfrak{o}_{i}\right) \leq 2\left(2 \operatorname{dim}_{E_{i}} V^{i}\right)$, and $m_{i}^{\prime} \in \mathbb{Z}$ such that

$$
\begin{aligned}
s_{i}\left(c_{i}\right)+\mathfrak{b}_{-m+}^{i i} & \subset \mathfrak{b}_{-m_{i}^{\prime}}^{\prime i i}, \\
m_{i}^{\prime} / e\left(\Lambda^{\prime(i)} \mid \mathfrak{o}_{i}\right) & <m / e\left(\Lambda^{(i)} \mid \mathfrak{o}_{i}\right) .
\end{aligned}
$$

[The extra factor 2 compared to loc. cit. in the bound for the period of $\Lambda^{\prime(i)}$ comes from the assumption that " $d=1$ " for the duality on on all self-dual lattice sequences (see $\$ 1.2$ ). In any case, the period $e\left(\Lambda^{\prime(i)} \mid \mathfrak{o}_{F}\right) \leq 4 \operatorname{dim}_{E_{i}} V^{i} e\left(E_{i} / F\right) \leq 4 N$.]

We put $\Lambda^{\prime}=\oplus_{i=1}^{l} \Lambda^{\prime(i)}, e^{\prime}=e\left(\Lambda^{\prime} \mid \mathfrak{o}_{F}\right), n^{\prime}=-\nu_{\Lambda^{\prime}}(\beta)$ and $m^{\prime}=e^{\prime} \sup \left\{m_{i}^{\prime} / e\left(\Lambda^{\prime(i)} \mid \mathfrak{o}_{F}\right)\right\}$ so that $\left[\Lambda^{\prime}, n^{\prime}, m^{\prime}, \beta\right]$ is a skew semisimple $\mathfrak{o}_{E^{-}}$-stratum. By Lemma 5.4. there exists $\theta_{-}^{\prime} \in \mathcal{C}_{-}\left(\Lambda^{\prime}, m^{\prime}, \beta\right)$ contained in $\pi$, and we also have $m^{\prime} / e^{\prime}<m / e$. As above, there now exist $\widetilde{\theta}_{-}^{\prime} \in \mathcal{C}_{-}\left(\Lambda^{\prime}, m^{\prime}-1, \beta\right)$ and $c \in \mathfrak{a}_{-m^{\prime}}^{\prime} \cap \mathcal{M}$ such that $\pi$ contains $\widetilde{\theta}_{-}^{\prime} \psi_{c}^{-}$.

The result now follows by iterating the above process, which will end either with $m^{\prime}=0$, contradicting the assumption on $\pi$, or with a fundamental derived stratum as required. Note that the iteration will terminate since the rational $m / e$ is strictly decreasing each time, while its denominator is bounded by, for example, $(4 N)$ !.

Hence we may take our pair $\left([\Lambda, n, m, \beta], \theta_{-}\right)$, with $\theta_{-}$contained in $\pi$, such that $\pi$ contains $\vartheta=$ $\widetilde{\theta}_{-} \psi_{c}^{-}$, for some $c \in \mathfrak{a}_{-m}^{-}$, with $\left[\Lambda_{\mathfrak{o}_{E}}, m, m-1, s(c)\right]$ fundamental. Moreover, since $e /(m, e)$ is bounded, where $e=e\left(\Lambda \mid \mathfrak{o}_{F}\right)$, we may take such a pair with $m / e$ minimal.

Now suppose that our fundamental stratum $\left[\Lambda_{\mathfrak{o}_{E}}, m, m-1, s(c)\right]$ is non- $G$-split, in the sense that none of the $\left[\Lambda_{\mathfrak{o}_{i}}, m, m-1, s_{i}\left(c_{i}\right)\right]$ are $G$-split. Then, by [14] (4.4), for $i=1, \ldots, l$, there exists a semisimple skew stratum $\left[\Lambda_{\mathfrak{o}_{i}}^{\prime(i)}, m_{i}^{\prime}, m_{i}^{\prime}-1, \alpha_{i}^{\prime}\right]$ in $B^{i i}$ such that

$$
\begin{aligned}
& s_{i}\left(c_{i}\right)+\mathfrak{b}_{-m+}^{i i} \subset \alpha_{i}^{\prime}+\mathfrak{b}_{-m_{i}^{\prime}}^{\prime i i}, \\
& m / e\left(\Lambda^{(i)} \mid \mathfrak{o}_{i}\right)=m_{i}^{\prime} / e\left(\Lambda^{\prime(i)} \mid \mathfrak{o}_{i}\right), \\
& \Lambda^{\prime(i)} \text { is a refinement of } \Lambda^{(i)} .
\end{aligned}
$$

As above, we put $\Lambda^{\prime}=\oplus_{i=1}^{l} \Lambda^{\prime(i)}, e^{\prime}=e\left(\Lambda^{\prime} \mid \mathfrak{o}_{F}\right)$ and $m^{\prime}=m_{i}^{\prime} e^{\prime} / e\left(\Lambda^{\prime(i)} \mid \mathfrak{o}_{F}\right)$. Note that the final two conditions imply that $\mathfrak{a}_{-m^{\prime}}^{\prime} \cap \mathcal{M} \subset \mathfrak{a}_{-m} \cap \mathcal{M}$, so that $\alpha^{\prime} \in \mathfrak{b}_{-m^{\prime}}^{\prime} \subset \mathfrak{b}_{-m}$. 
Now we may apply Lemma 5.4 once again to conclude that there exist $\widetilde{\theta^{\prime}} \in \mathcal{C}_{-}\left(\Lambda^{\prime}, m^{\prime}-1, \beta\right)$ and $c^{\prime} \in \mathfrak{a}_{-m^{\prime}}^{\prime}$ such that $s\left(c^{\prime}\right)=\alpha^{\prime}$ and $\pi$ contains the character $\vartheta^{\prime}:=\theta_{-}^{\prime} \psi_{c^{\prime}}^{-}$of $H_{-}^{m^{\prime}}\left(\beta, \Lambda^{\prime}\right)$. However, $\left[\Lambda^{\prime}, n^{\prime}, m^{\prime}-1, \beta+c^{\prime}\right]$ is equivalent to a semisimple stratum $\left[\Lambda^{\prime}, n^{\prime}, m^{\prime}-1, \beta^{\prime}\right]$, by Lemma 3.5, and $\widetilde{\theta_{-}^{\prime}} \psi_{c^{\prime}}^{-} \in \mathcal{C}_{-}\left(\Lambda^{\prime}, m^{\prime}-1, \beta^{\prime}\right)$. But we have $\left(m^{\prime}-1\right) / e\left(\Lambda^{\prime} \mid \mathfrak{o}_{F}\right)<m / e\left(\Lambda \mid \mathfrak{o}_{F}\right)$ and (using Proposition 5.5 if necessary to obtain a fundamental derived stratum) this contradicts the minimality of $\mathrm{m} / e$.

Hence we must have that the derived stratum $\left[\Lambda_{\mathfrak{o}_{E}}, m, m-1, s(c)\right]$ is $G$-split; we suppose, without loss of generality, that $\left[\Lambda_{\mathfrak{o}_{1}}^{\prime}, m^{\prime}, m^{\prime}-1, s_{1}\left(c_{1}\right)\right]$ is $G$-split. As in [14 $\S 2.1$, this gives rise to a decomposition of $E_{1}$-vector spaces $V^{1}=V_{0}^{1} \perp\left(V_{1}^{1} \oplus V_{-1}^{1}\right)$, where $V_{1}^{1}$ and $V_{-1}^{1}$ are totally isotropic with respect to $h$, such that $\Lambda(k)=\oplus_{-1 \leq j \leq 1}\left(\Lambda(k) \cap V_{j}^{1}\right)$ and $s(c) V_{j}^{1} \subset V_{j}^{1}$, for $j=-1,0,1$. Note, however, that we do not necessarily have $c_{1} V_{j}^{1} \subset V_{j}^{1}$, for $j=-1,0,1$. We show that we may in fact assume that this is the case.

For $j, k \in\{-1,0,1\}$, we write $A_{j k}^{(1)}$ for the space $\operatorname{Hom}_{F}\left(V_{k}^{1}, V_{j}^{1}\right)$, and, for $a \in A^{(11)}$, we will write $a=\sum_{j, k} a_{j k}$, with $a_{j k} \in A_{j k}^{(1)}$. For $j \neq k$ we have $s_{1}\left(c_{1}\right)_{j k}=0$ so $\left(c_{1}\right)_{j k} \in \mathfrak{a}_{-m}^{-} \cap a_{\beta}\left(A_{j k}^{(1)}\right)$. In particular, by 3.8 , there exists $x \in \mathfrak{m}_{m}^{(11)-}$ such that $\left(c_{1}\right)_{j k}=-a_{\beta}(x)_{j k}$. But, by Lemma 5.2. $C(x)$ normalizes $H_{-}^{m}$ and we have $\vartheta^{C(x)}=\widetilde{\theta}_{-} \psi_{c^{\prime}}^{-}$, where $c^{\prime}=c+a_{\beta}(x)$. This character certainly occurs in $\pi$ and we have $s\left(c^{\prime}\right)=s(c)$ and $c^{\prime} V_{j}^{1} \subset V_{j}^{1}$, for $j=-1,0,1$, as required.

We are now in the situation of $\S 4$ and, by Theorem $4.9, \pi$ is not supercuspidal, a contradiction. This completes the proof of Theorem 5.1 .

Remark 5.6. We have shown that any positive-level irreducible supercuspidal representation $\pi$ of $G$ contains a skew semisimple character $\theta_{-} \in \mathcal{C}_{-}(\Lambda, 0, \beta)$. Then $\pi$ certainly also contains the Heisenberg representation $\eta_{-}$of $J_{-}^{1}(\beta, \Lambda)$.

\subsection{Proof of Lemma 5.4}

We are now left to prove Lemma 5.4, for which we need some preliminary lemmas. All notation will be as in the previous section, so that $[\Lambda, n, m, \beta]$ is a skew semisimple stratum with associated decomposition $V=\bigoplus_{i=1}^{l} V^{i}$ and $\pi$ is an irreducible positive-level supercuspidal representation of $G$ containing $\vartheta=\widetilde{\theta}_{-} \psi_{c}^{-}$, for some $\widetilde{\theta}_{-} \in \mathcal{C}_{-}(\Lambda, m-1, \beta)$ and $c \in \mathfrak{a}_{-m}^{-} \cap \mathcal{M}$. For $t>0$, we put

$$
\begin{aligned}
\mathfrak{k}_{1}^{t}(\Lambda) & =\bigoplus_{i \neq j} \mathfrak{a}_{\frac{t}{2}+}^{i j} \oplus \bigoplus_{i} \mathfrak{a}_{t}^{i i}, & K_{1}^{t}(\Lambda) & =1+\mathfrak{k}_{1}^{t}(\Lambda), \\
\mathfrak{k}_{2}^{t}(\Lambda) & =\bigoplus_{i \neq j} \mathfrak{a}_{\frac{t}{2}}^{i j} \oplus \bigoplus_{i} \mathfrak{a}_{t}^{i i}, & K_{2}^{t}(\Lambda) & =1+\mathfrak{k}_{2}^{t}(\Lambda),
\end{aligned}
$$

and $K_{i,-}^{t}=K_{i,-}^{t}(\Lambda)=K_{i}^{t}(\Lambda) \cap G$, for $i=1,2$. We also put $H_{i}^{t}=H(\beta, \Lambda) \cap K_{i}^{t}$, for $i=1,2$, and define $J_{i}^{t}, H_{i,-}^{t}$ and $J_{i,-}^{t}$ similarly. We observe that we have $H_{1}^{m}=H_{2}^{m}$, and similarly for $J$.

Let $\vartheta=\widetilde{\theta}_{-} \psi_{c}^{-}$, as in the previous section, and let $\widetilde{\vartheta}$ denote the extension of $\vartheta$ to $H_{1,-}^{m}$ which is trivial on the unipotent parts - that is, $\widetilde{\vartheta}=\widetilde{\theta}_{-} \psi_{c}^{-}$, where $\widetilde{\theta}_{-}$is now extended to a simple character (also denoted $\widetilde{\theta}_{-}$) of $H^{\frac{m}{2}+}$.

Lemma 5.7. $\pi$ contains $\widetilde{\vartheta}$.

Proof We show that $\pi$ contains the character $\widetilde{\theta}_{-} \psi_{c^{\prime}}^{-}$of $H_{-}^{\frac{m}{2}+}$, for some $c^{\prime} \in \mathfrak{a}_{-m}^{-} \cap \mathcal{M}$ such that $c \equiv c^{\prime}\left(\bmod \left(\mathfrak{H}^{m}\right)^{*}\right)$. Then certainly $\widetilde{\vartheta}=\widetilde{\theta}_{-} \psi_{c^{\prime}}^{-} \mid H_{1,-}^{m}$ is contained in $\pi$. 
Since $H_{-}^{\frac{m}{2}+} / H_{-}^{m}$ is abelian and $\vartheta$ extends to a character $\widetilde{\theta}_{-} \psi_{c}^{-}$of $H_{-}^{\frac{m}{2}+}, \pi$ certainly contains some character of $H_{-}^{\frac{m}{2}}+$ extending $\vartheta$, of the form

$$
\vartheta_{1}=\widetilde{\theta}_{-} \psi_{c_{1}}^{-}, \quad \text { for some } c_{1} \in \mathfrak{a}_{-m} \text { with } c_{1} \equiv c \quad\left(\bmod \left(\mathfrak{H}^{m}\right)^{*}\right)
$$

Now, since $c \in \mathcal{M}$, for $i \neq j$ we have $\left(c_{1}\right)_{i j} \in\left(\mathfrak{H}^{m}\right)^{*}$. By Lemma 3.17, there exists $x \in \mathfrak{m}_{m-1}$ such that $a_{\beta}(x)_{i j}=-\left(c_{1}\right)_{i j}$, for $i \neq j$. By Corollary 3.20. $C(x)$ normalizes $H_{-}^{\frac{m}{2}+}$ and we have

$$
\vartheta_{2}:=\vartheta_{1}^{C(x)}=\widetilde{\theta}_{-} \psi_{c_{2}}^{-}, \quad \text { where } \quad c_{2}=c_{1}+C(x)^{-1}\left(\beta+c_{1}\right) C(x)-\left(\beta+c_{1}\right)
$$

A simple calculation shows that $c_{2} \equiv c_{1}+a_{\beta}(x)\left(\bmod \left(\mathfrak{H}^{m-1}\right)^{*}\right)$ so $\left(c_{2}\right)_{i j} \in\left(\mathfrak{H}^{m-1}\right)^{*}$, for $i \neq j$.

Since $\vartheta_{2}$ certainly occurs in $\pi$, we can iterate this process until, with $m^{\prime}=\left\lfloor\frac{m+1}{2}\right\rfloor$, we get $\vartheta_{m^{\prime}}=$ $\widetilde{\theta}_{-} \psi_{c_{m^{\prime}}}^{-}$in $\pi$, with $\left(c_{m^{\prime}}\right)_{i j} \in\left(\mathfrak{H}^{\frac{m}{2}+}\right)^{*}$, for $i \neq j$. Now, putting $c^{\prime}=\sum_{i=1}^{l}\left(c_{m^{\prime}}\right)_{i i} \in \mathcal{M}$, we see that $\pi$ contains $\vartheta_{m^{\prime}}=\widetilde{\theta}_{-} \psi_{c^{\prime}}^{-}$, as required.

Proposition 5.8 (cf. [6] (8.1.7)). Let $\mathfrak{G}$ be an open subgroup of $K_{2,-}^{m}$ and $\rho$ an irreducible representation of $\mathfrak{G}$ such that $\rho \mid H_{1,-}^{m}(\beta, \Lambda) \cap \mathfrak{G}$ contains $\widetilde{\vartheta} \mid H_{1,-}^{m}(\beta, \Lambda) \cap \mathfrak{G}$. Then $\pi \mid \mathfrak{G}$ contains $\rho$.

Proof We know, from Lemma 5.7, that $\pi$ contains $\widetilde{\vartheta}$. Now the proof is identical to that of [6] (8.1.7), given the following lemma:

Lemma 5.9 (cf. [6] (8.1.8)). There is a unique irreducible representation $\tau$ of $K_{-}^{m}$ such that $\tau \mid H_{1,-}^{m}(\beta, \Lambda)$ contains $\widetilde{\vartheta}$.

Proof By Corollary 3.12(iii), the commutator $\left[J_{1}^{m}, J_{1}^{m}\right] \subset H^{m+}$, so the pairing

$$
\begin{gathered}
\boldsymbol{k}_{\widetilde{\vartheta}}: J_{1,-}^{m} / H_{1,-}^{m} \times J_{1,-}^{m} / H_{1,-}^{m} \rightarrow \mathbb{C}^{\times}, \\
\boldsymbol{k}_{\widetilde{\vartheta}}(x, y)=\widetilde{\vartheta}[x, y],
\end{gathered}
$$

depends only on $\widetilde{\vartheta} \mid H_{-}^{m+}=\theta_{-}$. By Proposition $3.28, \boldsymbol{k}_{\widetilde{\theta}_{-}}$on $J^{\frac{m}{2}+} / H^{\frac{m}{2}+}$ is nondegenerate and since, from the simple case, $\boldsymbol{k}_{\widetilde{\theta}_{-}}$is nondegenerate on $J^{\frac{n}{2}}+\cap M / H^{\frac{m}{2}}+\cap M$, it follows that $\boldsymbol{k}_{\widetilde{\vartheta}}$ is also nondegenerate. Hence there is a unique irreducible representation $\mu$ of $J_{1,-}^{m}$ containing $\widetilde{\vartheta}$.

The result now follows once we have shown that the intertwining of $\mu$ in $K_{2,-}^{m}$ is contained in $J_{1,-}^{m}$, for then $\tau:=\operatorname{Ind}{ }_{J_{1,-}^{m}-}^{K_{2}^{m}} \mu$ is irreducible and is as required.

Since the restriction of $\mu$ to $H_{2,-}^{m+}$ is actually a multiple of $\widetilde{\theta}_{-} \mid H_{2,-}^{m+}$, it is enough to show that the intertwining of $\widetilde{\theta}_{-} \mid H_{2,-}^{m+}$ in $K_{2,-}^{m}$ is contained in $J_{1,-}^{m}=J_{2,-}^{m}$. Moreover, using Glauberman's correspondence as usual, we have $\widetilde{\theta}_{-}=\mathbf{g}(\widetilde{\theta})$, for some $\widetilde{\theta} \in \mathcal{C}^{\Sigma}\left(\Lambda, \frac{m}{2}, \beta\right)$ and it is enough to show that the intertwining in $K_{2}^{m}$ of the character $\widetilde{\theta} \mid H_{2}^{m+}$ is contained in $J$.

So let $g \in K_{2}^{m}$ intertwine $\widetilde{\theta} \mid H_{2}^{m+}$; then certainly $g$ intertwines the simple character $\widetilde{\theta} \mid H^{m+}$ so, by Theorem 3.22 , we have $g \in \Gamma_{m} B_{\beta}^{\times} \Gamma_{m} \cap K_{2}^{m}$. Since $\Gamma_{m} K_{2}^{m} \Gamma_{m} \cap B_{\beta}^{\times} \subset J$ normalizes $\widetilde{\theta}$, we need only consider $g \in \mathrm{T}_{m} \cap K_{2}^{m}$. We write $g=1+x$, with $x \in \mathfrak{m}_{m}$, and use the Iwahori decomposition $g=l_{g} m_{g} u_{g}$, with $l_{g} \in N_{l}, m_{g} \in M, u_{g} \in N_{u}$. We also write $l_{g}=1+x_{l}, m_{g}=1+x_{m}$ and $u_{g}=1+x_{u}$, with $x_{l} \in \mathfrak{m}_{m} \cap A_{l}, x_{m} \in \mathfrak{m}_{m} \cap \mathcal{M}$ and $x_{u} \in \mathfrak{m}_{m} \cap A_{u}$. 
We proceed now in a very similar way to the proofs of Theorems 3.22 and 4.2 . Set

$$
\mathfrak{k}_{l}=\mathfrak{H}^{m+}+\mathfrak{H}^{\frac{m}{2}+} \cap A_{u}, \quad K_{l}=1+\mathfrak{k}_{l} .
$$

$g$ certainly intertwines $\widetilde{\theta}$ on $K_{l}$ so, by Corollary 3.20 , we have

$$
g^{-1}\left(\beta+\mathfrak{k}_{l}^{*}\right) g \cap\left(\beta+\mathfrak{k}_{l}^{*}\right) \neq \emptyset .
$$

Now $a_{\beta}\left(x_{m}\right)$ and $a_{\beta}\left(x_{u}\right) \in \mathfrak{k}_{l}^{*}$, by Lemma 3.17 so we see that this implies $a_{\beta}\left(x_{l}\right) \in \mathfrak{k}_{l}^{*}$ also. This is all really happening in $A_{l}$, where $\mathfrak{k}_{l}^{*} \cap A_{l}=\left(\mathfrak{H}^{\frac{m}{2}}+\right)^{*} \cap A_{l}$ so, again by Lemma 3.17 , we find that there exists $y_{l} \in \mathfrak{m} \frac{m}{2}$ such that $a_{\beta}\left(y_{l}\right)=\alpha\left(x_{l}\right)$. Then, by injectivity of $a_{\beta}$ on $A_{l}$, we see that $x_{l}=y_{l}$ so that $g_{l} \in \Gamma_{\frac{m}{2}}$. But $\frac{m}{2}<\frac{r}{2}$ so $\Gamma_{\frac{m}{2}} \subset J$ and $g_{l}$ normalizes $\widetilde{\theta}$.

Hence we may assume $g=g_{m} g_{u}$ and, repeating with $\mathfrak{k}_{u}=\mathfrak{H}^{m+}+\mathfrak{H}^{\frac{m}{2}+} \cap A_{l}$, we see we may assume $g \in \Gamma_{m} \cap M$. Now the result follows from the simple case in the proof of [6] (8.1.8).

This also completes the proof of Proposition 5.8 .

Now we are in a position to prove Lemma 5.4 with the additional hypothesis:

$$
U^{m^{\prime}}\left(\Lambda^{\prime}\right) \subset K_{2}^{m}(\Lambda)
$$

From the simple case (see [6] $\S 1.3$ ), we may choose $c^{\prime} \in \mathfrak{a}_{-m^{\prime}}^{\prime-} \cap \mathfrak{a}_{-m}^{-} \cap \mathcal{M}$ such that $s\left(c^{\prime}\right)=\alpha^{\prime}$. (Note that we may take $c^{\prime}=0$ if $\left.\alpha^{\prime}=0\right)$. Put $\delta=c^{\prime}-c$; then, since $s(c)+\mathfrak{b}_{-m+} \subset s\left(c^{\prime}\right)+\mathfrak{b}_{-m^{\prime}+}^{\prime}$, we have

$$
\delta \in\left(\mathfrak{a}_{1-m^{\prime}}^{\prime-}+a_{\beta}(A)\right) \cap \mathfrak{a}_{-m}^{-} \cap \mathcal{M}=\mathfrak{a}_{1-m^{\prime}}^{\prime-} \cap \mathfrak{a}_{-m}^{-} \cap \mathcal{M}+a_{\beta}(A) \cap \mathfrak{a}_{-m}^{-} \cap \mathcal{M},
$$

where the equality of lattices is from the simple case [6] (8.1.13). Then, again by the simple case [6] (1.4.10), there exists $x \in \mathfrak{n}_{m} \cap \mathfrak{a}_{r-m}^{-}$such that

$$
\delta-a_{\beta}(x) \in \mathfrak{a}_{1-m^{\prime}}^{-} \cap \mathfrak{a}_{-m}^{-} \cap \mathcal{M} .
$$

$C(x)$ normalizes $H_{-}^{m}(\beta, \Lambda)$ and $\vartheta^{C(x)}=\vartheta \psi_{a_{\beta}(x)}^{-}$, by Lemma 5.2 . Now, from the transfer property, there exists $\theta_{-}^{\prime} \in \mathcal{C}_{-}\left(\Lambda^{\prime}, m^{\prime}-1, \beta\right)$ such that $\theta_{-}$and $\theta_{-}^{\prime}$ agree where they are both defined. Then, on $H_{-}^{m}(\beta, \Lambda) \cap H_{-}^{m^{\prime}}\left(\beta, \Lambda^{\prime}\right)$, we have

$$
\vartheta^{C(x)}=\theta_{-} \psi_{c}^{-} \psi_{\delta}=\theta_{-}^{\prime} \psi_{c^{\prime}}^{-}
$$

Moreover, since $C(x) \in M, \widetilde{\vartheta}^{C(x)}$ and $\theta_{-}^{\prime} \psi_{c^{\prime}}^{-}$are trivial on the unipotent parts $N_{u}$ and $N_{l}$ so that $\widetilde{\vartheta}^{C}(x)$ and $\theta_{-}^{\prime} \psi_{c^{\prime}}^{-}$agree where they are both defined. Finally, since $H^{m^{\prime}}\left(\beta, \Lambda^{\prime}\right) \subset U^{m^{\prime}}\left(\Lambda^{\prime}\right)$ which, by hypothesis $(H)$, is included in $K_{2}^{m}(\Lambda)$, by Proposition 5.8 we have that $\pi$ contains $\vartheta^{\prime}:=\theta_{-}^{\prime} \psi_{c^{\prime}}^{-}$, as required.

For the general case we need some additional technical lemmas, which use the description of the building in terms of lattice functions from [2]. We refer the reader to op. cit. $\S \S 0-7$ for more details. The idea is that, if $(\mathrm{H})$ is not satisfied, we can pass more gradually from $\Lambda$ to $\Lambda^{\prime}$, via some intermediate sequences.

For $\Lambda, \Lambda^{\prime}$ two lattice sequences, there exists a common splitting - that is, an $F$-basis $\mathcal{B}$ of $V$ which splits them - which we fix. Choosing such a basis is the same thing as choosing an apartment in 
the (extended) affine building $\mathcal{I}^{1}(\widetilde{G}, F)$ of $\widetilde{G}$. Indeed, by [2] Propositions 1.4, 2.4, there is a (unique upto translation) $G$-set isomorphism between $\mathcal{I}^{1}(\widetilde{G}, F)$ and the set of lattice functions on $V$, of which, by op. cit. $\S 7$, the lattice sequences are the subset of "rational points" (i.e. barycentres of vertices with rational weights). By transferring the structure from the building, we then have an affine structure on the set of lattice sequences.

The choice of a basis of $V$ (or apartment in $\left.\mathcal{I}^{1}(\widetilde{G}, F)\right)$ gives us a set of $\operatorname{rots}\left\{\alpha_{i j}\right\},(1 \leq i, j \leq N$, $i \neq j)$ by $\alpha_{i j}\left(\operatorname{diag}\left(u_{1}, \ldots, u_{N}\right)\right)=u_{j} u_{i}^{-1}$. We also set $\alpha_{i i}$ to be the "zero root", for each $i$. Then, if $x \in \mathcal{I}^{1}(\widetilde{G}, F)$ corresponds to $\Lambda$, [2] Corollaries 4.5, 4.6 say that, for $r \in \mathbb{R}, \mathfrak{a}_{r}(\Lambda)$ is the set of matrices $\left(y_{i j}\right) \in M(N, F) \simeq A$ satisfying

$$
y_{i j} \in \mathfrak{p}_{F}^{\left\lceil\left(\frac{r}{e}-\alpha_{i j}(x)\right)\right\rceil}, \quad i, j=1 \ldots N
$$

where $e=e(\Lambda)$. This does not depend on the choice of the identification between $\mathcal{I}^{1}(\widetilde{G}, F)$ and the set of lattice functions.

Lemma 5.11. Let $[\Lambda, m, m-1, b],\left[\Lambda^{\prime}, m^{\prime}, m^{\prime}-1, b^{\prime}\right]$ be strata in $A$ and put $e=e(\Lambda), e^{\prime}=e\left(\Lambda^{\prime}\right)$. Suppose that

$$
b+\mathfrak{a}_{-m+}(\Lambda) \subset b^{\prime}+\mathfrak{a}_{-m^{\prime}+}\left(\Lambda^{\prime}\right), \quad b^{\prime} \in \mathfrak{a}_{-m}(\Lambda) .
$$

Let $t$ be a rational number and put

$$
\Lambda_{t}=(1-t) \Lambda+t \Lambda^{\prime}, \quad e_{t}=e(\Lambda), \quad m_{t}=e_{t}\left((1-t) \frac{m}{e}+t \frac{m^{\prime}}{e^{\prime}}\right) .
$$

Then $b, b^{\prime} \in \mathfrak{a}_{-m_{t}}\left(\Lambda_{t}\right)$, for all $0 \leq t \leq 1$, and, for $0 \leq s \leq t \leq 1$ rational numbers,

$$
\mathfrak{a}_{-m_{s}+}\left(\Lambda_{s}\right) \subset \mathfrak{a}_{-m_{t}+}\left(\Lambda_{t}\right)
$$

Note that, in this lemma, we are just looking at the line segment $\left[\Lambda, \Lambda^{\prime}\right]$ in the building.

Proof For $r \in \mathbb{R}$, we abbreviate $\mathfrak{a}_{r}=\mathfrak{a}_{r}(\Lambda)$ and $\mathfrak{a}_{r}^{\prime}=\mathfrak{a}_{r}\left(\Lambda^{\prime}\right)$. We note that, since $b^{\prime} \in \mathfrak{a}_{-m} \cap \mathfrak{a}_{-m^{\prime}}^{\prime}$, the same is also true for $b$ and $\mathfrak{a}_{-m+} \subset \mathfrak{a}_{-m^{\prime}+}^{\prime}$.

We let $x, x^{\prime}$ be the points in the building corresponding to $\Lambda, \Lambda^{\prime}$ respectively and, for $0 \leq t \leq 1$, let $x_{t}$ be the point corresponding to $\Lambda_{t}$. We also put $a_{i j}^{t}=\alpha_{i j}\left(x_{t}\right)$, for $1 \leq i, j \leq N$ and $0 \leq t \leq 1$, and we will use the description (5.10).

Since $b \in \mathfrak{a}_{-m} \cap \mathfrak{a}_{-m^{\prime}}^{\prime}$, we have, for $1 \leq i, j \leq N$,

$$
v_{F}\left(b_{i j}\right) \geq-\frac{m}{e}-a_{i j}^{0}, \quad v_{F}\left(b_{i j}\right) \geq-\frac{m^{\prime}}{e^{\prime}}-a_{i j}^{1} .
$$

But then $v_{F}\left(b_{i j}\right) \geq(1-t)\left(-\frac{m}{e}-a_{i j}^{0}\right)+t\left(-\frac{m^{\prime}}{e^{\prime}}-a_{i j}^{1}\right)=-\frac{m_{t}}{e_{t}}-a_{i j}^{t}$. Since $v_{F}\left(b_{i j}\right)$ is an integer, this means that $b \in \mathfrak{a}_{-m_{t}}\left(\Lambda_{t}\right)$, as required. The same is true for $b^{\prime}$.

For the last assertion, we observe that, since $m_{t}$ is an integer and every $a_{i j}^{t}$ can be written as a fraction with denominator $e_{t}, \mathfrak{a}_{-m_{t}+}\left(\Lambda_{t}\right)$ is the set of matrices $\left(y_{i j}\right) \in M(N, F) \simeq A$ satisfying

$$
y_{i j} \in \mathfrak{p}_{F}^{\left(-\frac{m_{t}}{e_{t}}-a_{i j}^{t}\right)+}, \quad i, j=1 \ldots N
$$


Since $\mathfrak{a}_{-m+} \subset \mathfrak{a}_{-m^{\prime}+}^{\prime}$, we have

$$
\left(-\frac{m}{e}-a_{i j}^{0}\right)+\geq\left(-\frac{m^{\prime}}{e^{\prime}}-a_{i j}^{1}\right)+
$$

But we certainly have that $\left(-\frac{m_{t}}{e_{t}}-a_{i j}^{t}\right)$ lies in the interval whose endpoints are $\left(-\frac{m}{e}-a_{i j}^{0}\right)$ and $\left(-\frac{m^{\prime}}{e^{\prime}}-a_{i j}^{1}\right)$, and the result follows easily.

Now, if $x, x^{\prime}$ are points in the building corresponding to $\Lambda, \Lambda^{\prime}$ respectively, we put

$$
\left|\Lambda-\Lambda^{\prime}\right|:=\max _{1 \leq i, j \leq N}\left|\alpha_{i j}(x)-\alpha_{i j}\left(x^{\prime}\right)\right| .
$$

Again, this does not depend on the choice of the identification between $\mathcal{I}^{1}(\widetilde{G}, F)$ and the set of lattice functions.

Lemma 5.12. Let $k>0$. Then there exists $\delta>0$ such that, for all lattice sequences $\Lambda, \Lambda^{\prime}$ split by $\mathcal{B}$ with $\left|\Lambda-\Lambda^{\prime}\right|<\delta$, we have $\mathfrak{a}_{k e}(\Lambda) \subset \mathfrak{a}_{\frac{k e^{\prime}}{2}+}\left(\Lambda^{\prime}\right)$, where $e=e(\Lambda)$ and $e^{\prime}=e\left(\Lambda^{\prime}\right)$.

Proof We just take $\delta=\frac{k}{2}$ and the result is clear from 5.10.

We can now complete the proof of Lemma 5.4 in the general case (i.e. without the hypothesis $(\mathrm{H})$ ). We put $\alpha=s(c), k=\min \left\{\frac{m}{e}, \frac{m^{\prime}}{e^{\prime}}\right\}$ and choose $\delta$ as in Lemma 5.12. Let $q$ be an integer greater than $\frac{1}{\delta}\left|\Lambda-\Lambda^{\prime}\right|$ and, for each integer $t$ with $0 \leq t \leq q$, let $\Lambda_{t}$ be the lattice sequence $\left(1-\frac{t}{q}\right) \Lambda+\frac{t}{q} \Lambda^{\prime}$. (This was denoted $\Lambda_{t / q}$ in Lemma 5.11.). Note that each $\Lambda_{t}$ is an $\mathfrak{o}_{E}$-lattice sequence, since $\Lambda$ and $\Lambda^{\prime}$ are. By Lemma 5.11 applied to these $\mathfrak{o}_{E}$-lattice sequences, we have that, for $0 \leq t<q$,

(i) $\alpha \in \mathfrak{b}_{-m_{t}}^{-}\left(\Lambda_{t}\right)$;

(ii) $\mathfrak{b}_{-m_{t}+}\left(\Lambda_{t}\right) \subset \mathfrak{b}_{-m_{t+1}+}\left(\Lambda_{t+1}\right)$.

Moreover, by Lemma 5.12, we also have

(iii) $U^{m_{t+1}}\left(\Lambda_{t+1}\right) \subset U^{\frac{m_{t}}{2}+}\left(\Lambda_{t}\right)$.

Taking the dual of (ii), we see that $\mathfrak{b}_{m_{t+1}}\left(\Lambda_{t+1}\right) \subset \mathfrak{b}_{m_{t}}\left(\Lambda_{t}\right)$. Now, by choosing a common "generalized (W,E)-decomposition" for $\Lambda_{t}, \Lambda_{t+1}$, we see (as in [6] top of page 272) that we also have $\mathfrak{a}_{m_{t+1}}\left(\Lambda_{t+1}\right) \cap \mathcal{M} \subset \mathfrak{a}_{m_{t}}\left(\Lambda_{t}\right) \cap \mathcal{M}$. In particular, together with (iii) this implies

$$
U^{m_{t+1}}\left(\Lambda_{t+1}\right) \subset K_{2}^{m_{t}}\left(\Lambda_{t}\right)
$$

The result now follows by repeated application of the case where $(\mathrm{H})$ is satisfied.

\section{References}

[1] Blasco L., Description du dual admissible de $U(2,1)(F)$ par la théorie des types de C. Bushnell et P. Kutzko, Manuscripta Math. 107(2) (2002) 151-186.

[2] Broussous, P., Lemaire, B., Building of $\mathrm{GL}(m, D)$ and centralizers, Transform. Groups, 7(1) (2002) 15-50. 
[3] Broussous P., Stevens S., Buildings of classical groups and centralizers of Lie algebra elements, Manuscript, July 2003.

[4] Bushnell C.J., Henniart G., Local tame lifting for $G L(N)$ I: simple characters, Publ. Math. IHES 83 (1996) 105-233.

[5] Bushnell C.J., Henniart G., Local tame lifting for $G L(N)$ II: wildly ramified supercuspidals, Astérisque 254 (1999).

[6] Bushnell C.J., Kutzko P.C., The admissible dual of $G L(N)$ via compact open subgroups, Princeton University Press, 1993.

[7] Bushnell C.J., Kutzko P.C., Semisimple types, Compositio Math. 119 (1999) 53-97.

[8] Bushnell C.J., Kutzko P.C., Smooth representations of reductive p-adic groups: structure theory via types, Proc. London Math. Soc. (3) 77 (1998) 582-634.

[9] Glauberman G., Correspondences of characters for relatively prime operator groups, Canad. J. Math. (4) 20 (1968) 1465-1488.

[10] Secherre V., Représentations lisses de GL(m,D), I: caractères simples, Bull. Soc. Math. France, to appear.

[11] Stevens S., Types and supercuspidal representations of $p$-adic symplectic groups, Thesis, King's College London (1998).

[12] Stevens S., Double coset decompositions and intertwining, Manuscripta Math. 106(3) (2001) 349-364.

[13] Stevens S., Intertwining and supercuspidal types for classical $p$-adic groups, Proc. London Math. Soc. (3) 83(1) (2001) 120-140.

[14] Stevens S., Semisimple strata for p-adic classical groups, Ann. Sci. École Norm. Sup. (4) 35(3) (2002) 423-435.

[15] Yu J., Construction of tame supercuspidal representations, J. Amer. Math. Soc. (14(3) (2001) 579-622 (electronic).

Shaun Stevens

School of Mathematics

University of East Anglia

Norwich NR4 7TJ

United Kingdom

email: ginnyshaun@bigfoot.com 